\title{
THE ANTICIPATION OF LEISURE TIME
}

The Relationship between Pleasant Anticipation of a Planned Leisure Activity, Leisure Thoughts, General Anticipation of Leisure Time, and Employees' Work Engagement

\author{
Inaugural-Dissertation \\ in der Fakultät Humanwissenschaft \\ der Otto-Friedrich-Universität Bamberg
}

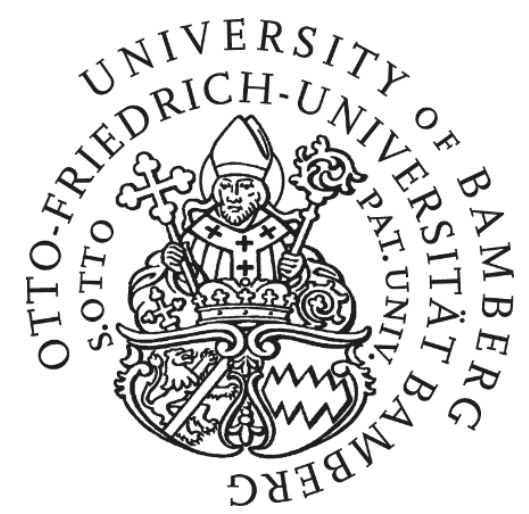

vorgelegt von

Sebastian Seibel

aus Saarbrücken

Bamberg, den 28.07.2021 
This work is available as a free online version via the Current Research Information System (FIS; fis.uni-bamberg.de) of the University of Bamberg. The work - with the exception of cover, quotations and illustrations - is licensed under the CC-License CC-BY-NC.

Lizenzvertrag: Creative Commons Namensnennung 4.0

http://creativecommons.org/licenses/by/4.0.

URN: urn:nbn:de:bvb:473-irb-530760

DOI: https://doi.org/10.20378/irb-53076

Tag der mündlichen Prüfung: 03.12.2021

Dekan:

Universitätsprofessor Dr. Jörg Wolstein

Betreuerin: Universitätsprofessorin Dr. Judith Volmer

Weiterer Gutachter: Universitätsprofessor Dr. Jörg Wolstein 


\section{ACKNOWLEDGEMENT}

First and foremost, I thank my advisor, Professor Judith Volmer, for her support and guidance throughout every step of this dissertation. I really appreciated the helpful, honest, fast, and sometimes challenging feedback that supported and motivated me very much in writing articles and conducting research. Furthermore, I am grateful for her trust in my dissertation topic and the opportunity to study a self-chosen research area.

I further thank my co-authors, Professor Antje Schmitt (Study 1) and Professor Christine Syrek (Study 2), for their support during their time as acting professors at the University of Bamberg. I learned much during insightful discussions and from detailed feedback on different manuscripts. Furthermore, I am thankful for their ongoing support and collaboration after they left the University of Bamberg.

Moreover, I want to thank Professor Jörg Wolstein for reviewing this dissertation and Professor Julia Franz and Professor Claus-Christian Carbon for participating in the dissertation committee.

In addition, I thank my former supervisors at the University of Wuerzburg, Professor Tanja Bipp, Professor Regina Kempen, and Professor Ingela Jöns, who provided me with time and autonomy to finish this dissertation.

Finally, I thank Stefanie Richter-Killenberg for her insightful feedback on the final draft of this dissertation. I also thank all my present and former colleagues from the University of Bamberg and the University of Wuerzburg for interesting discussions, providing feedback, sharing knowledge, and, of course, all the good times we had while working together.

Special thanks go to my parents, who always supported and encouraged me to finish this dissertation, and who patiently listened to all issues and doubts in the last six years. 


\section{STUDIES INCLUDED IN THIS DISSERTATION}

This dissertation is based on three studies that have been published in or submitted to peerreviewed, international scientific journals. The studies differ slightly from the published or submitted manuscripts in their formatting to better fit the outline of this dissertation, but their content has not been changed.

The included studies are the following:

Study 1: Seibel, S., Volmer, J., \& Schmitt, A. (2022). Pleasant anticipation of leisure time and work engagement: Findings from two studies. Leisure Science. (under review)

Study 2: Seibel, S., Volmer, J., \& Syrek, C. J. (2020). Get a taste of your leisure time: The relationship between leisure thoughts, pleasant anticipation, and work engagement. European Journal of Work and Organizational Psychology, 6(29), 889-906. https://doi.org/10.1080/1359432X.2020.1804875

Study 3: Seibel, S., \& Volmer, J. (2021). A diary study on anticipated leisure time, morning recovery, and employees' work engagement. International Journal of Environmental Research and Public Health,18, 9436. https://doi.org/10.3390/ijerph18189436 


\section{CONTRIBUTION TO THE STUDIES}

\section{Contribution to Study 1}

Sebastian Seibel developed the theoretical background and the hypotheses for this study; Sebastian Seibel, Antje Schmitt, and Judith Volmer planned the Study; Sebastian Seibel conducted the study and analysed the data; Sebastian Seibel wrote a first draft of the manuscript; Judith Volmer and Antje Schmitt provided feedback on earlier versions of the manuscript; Sebastian Seibel revised earlier versions of the manuscript; all authors have read the final version and agreed to the submitted version of the manuscript.

\section{Contribution to Study 2}

Sebastian Seibel developed the theoretical background and the hypotheses for this study; Sebastian Seibel, Christine J. Syrek, and Judith Volmer planned the Study; Sebastian Seibel conducted the study and analysed the data; Sebastian Seibel wrote a first draft of the manuscript; Judith Volmer and Christine J. Syrek provided feedback on earlier versions of the manuscript; Sebastian Seibel revised earlier versions of the manuscript; all authors have read the final version and agreed to the published version of the manuscript.

\section{Contribution to Study 3}

Sebastian Seibel developed the theoretical background and the hypotheses for this study; Sebastian Seibel and Judith Volmer planned the study; Sebastian Seibel conducted the study and analysed the data; Sebastian Seibel wrote a first draft of the manuscript; Judith Volmer provided feedback on earlier versions of the manuscript; Sebastian Seibel revised earlier versions of the manuscript; both authors have read the final version and agreed to the submitted version of the manuscript. 


\section{SUMMARY}

Recovery research has demonstrated that leisure time and leisure activities are crucial for employees' recovery, psychological well-being, general health, and job performance. Because leisure time offers opportunities to recover and gain new resources (e.g., energy), employees may also anticipate their leisure time while still working. Hence, I investigated in three studies whether the anticipation of leisure time is related to employees' work engagement, a positive, motivational work-related state associated with employees' well-being and job performance.

In Study 1, the focus was on pleasant anticipation of a planned leisure activity and work engagement. Pleasant anticipation is a positive, affective reaction because of a future event with a high probability of occurrence. I assumed that pleasant anticipation indicates an upcoming resource gain, and therefore, pleasant anticipation may positively relate to employees' work engagement. In addition, recovery-related self-efficacy (RRSE) was investigated as a moderator in this relationship. Two online studies were conducted: Study 1a $(N=87$ employees $)$ covered one working day, and Study $1 \mathrm{~b}(N=84$ employees $)$ contained five days within one working week. The results revealed that pleasant anticipation was positively related to work engagement between persons but not within persons. RRSE was not found to be a moderator.

Study 2 investigated whether employees think of their upcoming leisure time during the working day. Hence, the construct of leisure thoughts was introduced, and two types of leisure thoughts were differentiated, namely thoughts of leisure time (ToLT) and thoughts of a planned leisure activity (ToPLA). I argued that pleasant anticipation of a planned leisure activity relates to the frequency of ToPLA, yet not of ToLT, and that both types of leisure thoughts occur more frequently at the beginning and the end of the working day. Furthermore, as leisure thoughts may distract employees from the task at hand, they may negatively relate to work engagement across the working day and within one hour. However, ToPLA may also be positively associated with work engagement in the subsequent hour when the planned leisure activity has a positive leisure plan valence. In sum, the findings from a one-day online survey with hourly measurement points ( $N=89$ employees) supported the hypotheses for the occurrence of both types of leisure thoughts. Furthermore, ToLT were negatively related to work engagement across the working day, and ToPLA were negatively related to work engagement within the same hour. However, the relationship between ToPLA and work engagement in the subsequent hour was negative when the leisure plan valence was positive.

Based on the findings from Study 1 and Study 2, Study 3 shed light on leisure thoughts as a mechanism, which connects pleasant anticipation of a planned leisure activity and work 
engagement. Thus, ToPLA were examined as a mediator between pleasant anticipation and work engagement on a daily level. Furthermore, morning recovery was incorporated to ascertain whether recovery (i.e., a consequence of past leisure time) and the anticipation of leisure time simultaneously explain work engagement. In addition, because pleasant anticipation of a planned leisure activity only referred to a specific leisure activity, general anticipation of leisure time was introduced as a cognitive, overall evaluation of employees' entire leisure time after the working day. An online diary study $(N=148$ employees $)$ across one week was conducted. The results revealed that general anticipation of leisure time, yet not pleasant anticipation of a planned leisure activity, and morning recovery were positively related to work engagement within persons. Furthermore, pleasant anticipation of a planned leisure activity was positively related to ToPLA, but ToPLA was not related to work engagement; thus, ToPLA was not found to be a mediator.

Across three studies, different variables referring to the anticipation of leisure time were positively or negatively associated with work engagement, indicating that upcoming leisure time is crucial for present work engagement. The findings extend the understanding of leisure time, have practical implications for recovery training and work engagement interventions, and pave the way for further research on the anticipation of leisure time and leisure activities. 


\section{TABLE OF CONTENTS}

ACKNOWLEDGEMENT ............................................................................................II

STUDIES INCLUDED IN THIS DISSERTATION ...................................................IV

CONTRIBUTION TO THE STUDIES ..............................................................

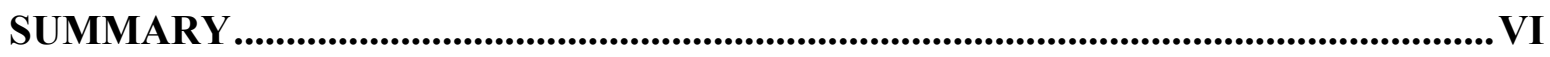

TABLE OF CONTENTS ........................................................................................

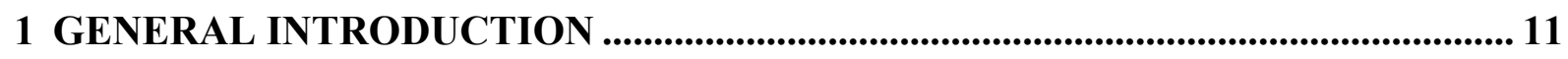

AIMS AND OUTLINE OF THE DISSERTATION .................................................................. 13

First Research Goal: The Relationship between the Anticipation of Leisure Time

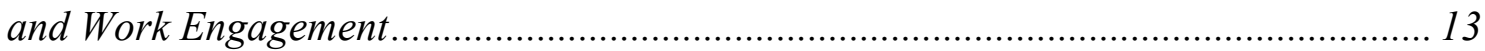

Second Research Goal: Antecedents and Consequences of Leisure Thoughts .............. 15

Third Research Goal: Extending and Replicating the Results from

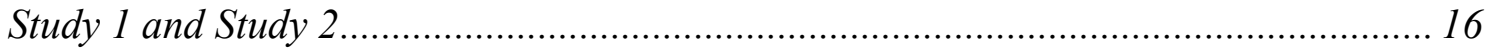

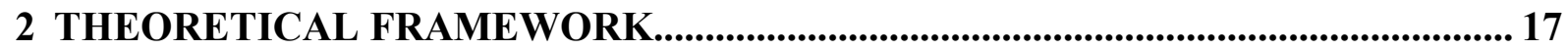

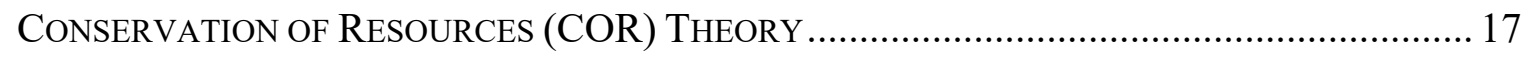

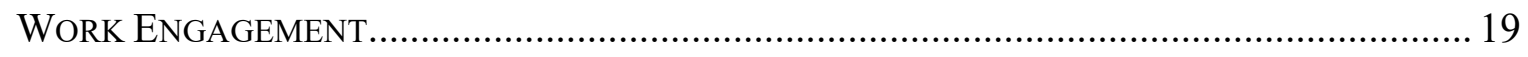

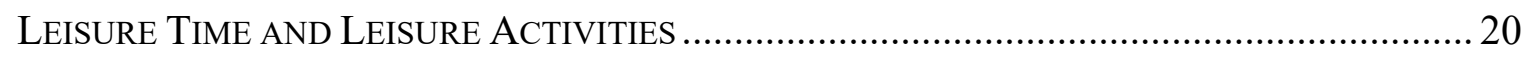

PleASANT ANTICIPATION OF A PLANNED LEISURE ACTIVITY .......................................... 21

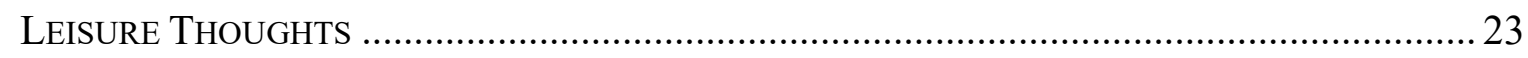

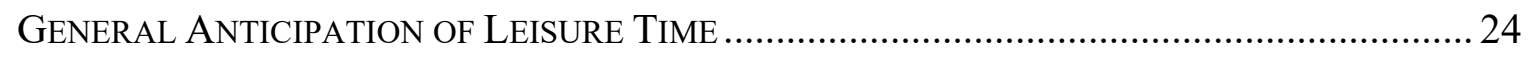

3 STUDY 1: PLEASANT ANTICIPATION OF LEISURE TIME AND WORK ENGAGEMENT: FINDINGS FROM TWO STUDIES......................................... 26

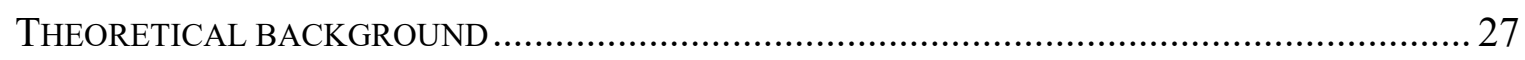

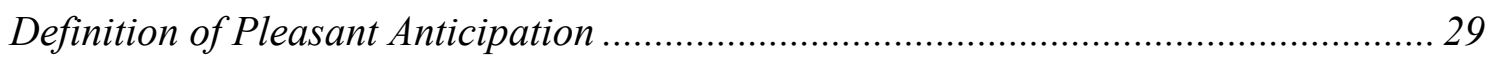

Pleasant Anticipation of a Planned Leisure Activity and Work Engagement ................ 29

Recovery-Related Self-Efficacy (RRSE) as a Moderator............................................ 31

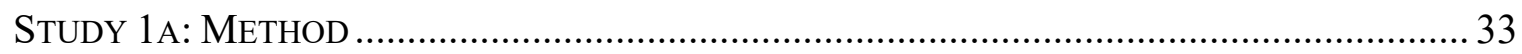

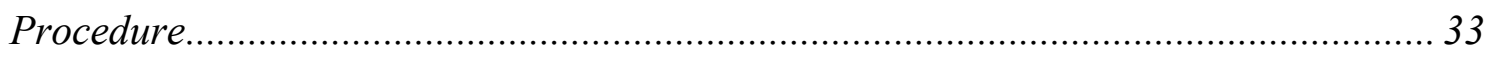

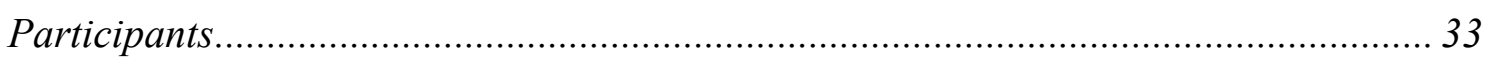

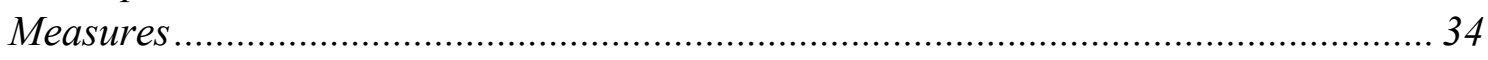

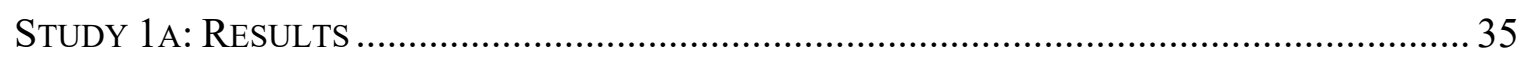

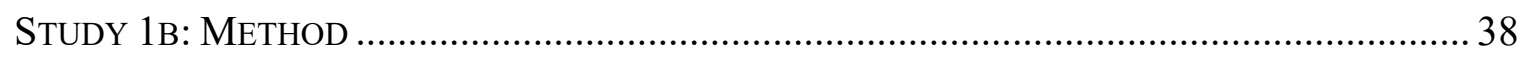

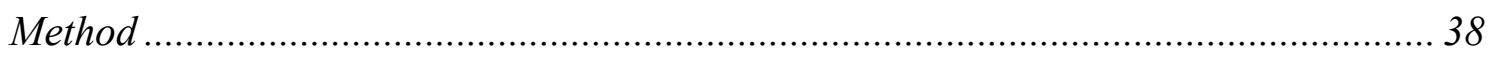

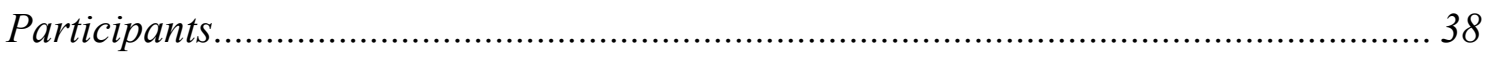

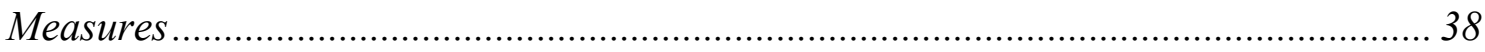

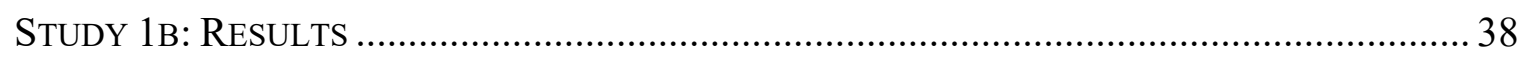

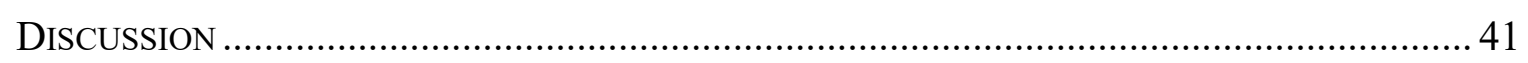

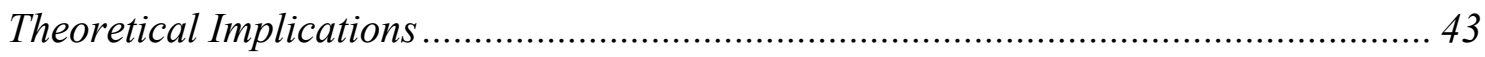

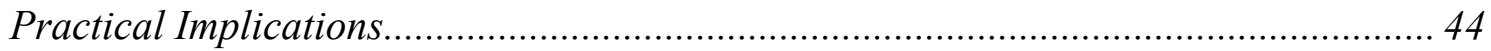

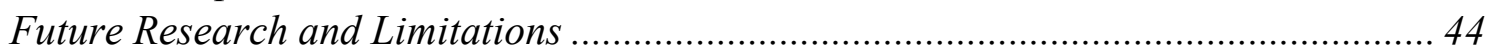




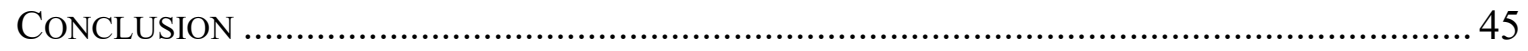

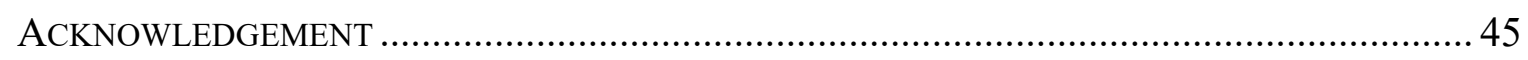

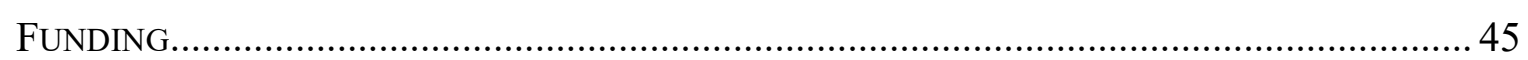

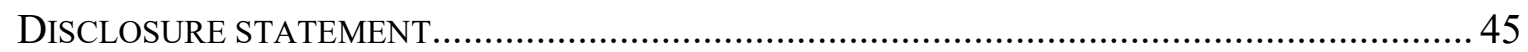

\section{STUDY 2: GET A TASTE OF YOUR LEISURE TIME - THE RELATIONSHIP} BETWEEN LEISURE THOUGHTS, PLEASANT ANTICIPATION, AND WORK

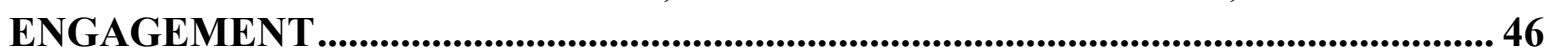

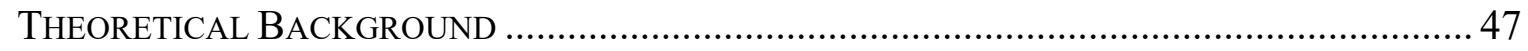

Definition of Leisure Time and Leisure Thoughts ........................................................... 48

Frequency of Leisure Thoughts During the Working Day .......................................... 50

Positive Relationship between Pleasant Anticipation and the Frequency of ToPLA ..... 51

Negative Relationship between Leisure Thoughts and Work Engagement .................... 53

The Moderating Role of Leisure Plan Valence .............................................................. 54

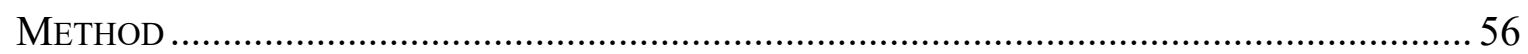

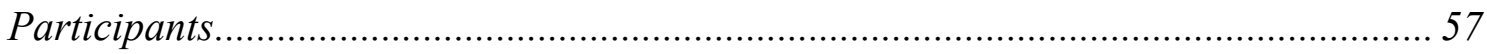

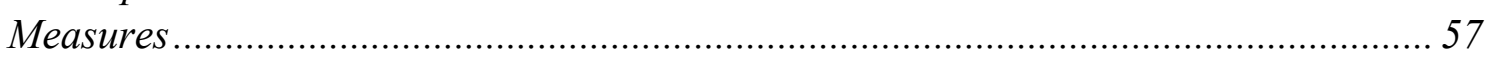

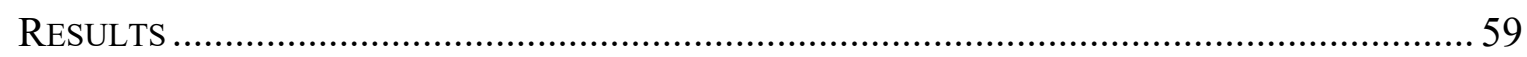

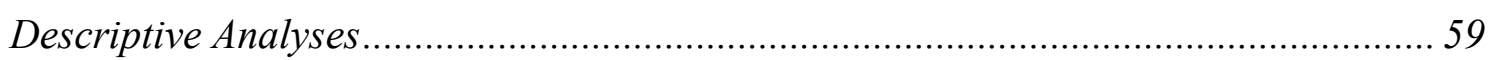

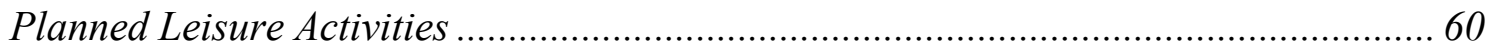

Time Trend for Leisure Thoughts and their Relationship with Pleasant Anticipation... 61

Negative Relationship between Leisure Thoughts and Work Engagement ................... 63

Relationship between ToPLA and Work Engagement for Different Levels

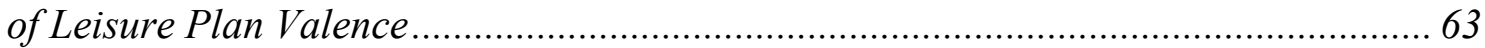

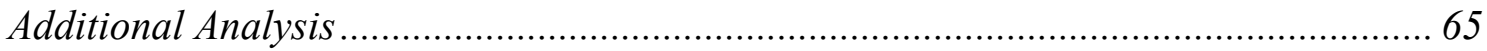

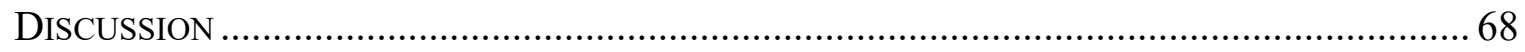

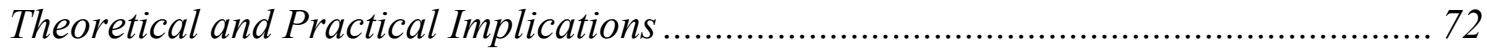

Limitations and Directions for Future Research ...................................................... 73

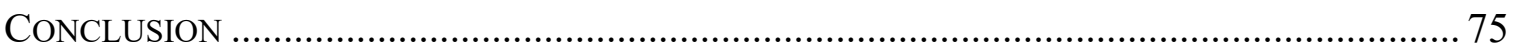

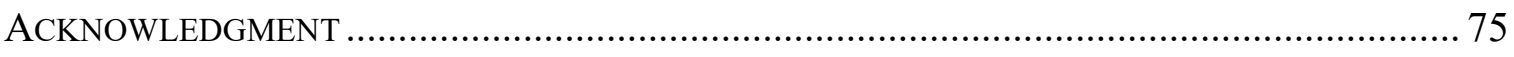

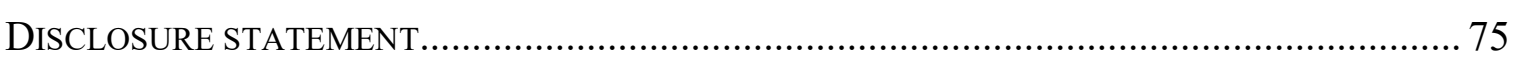

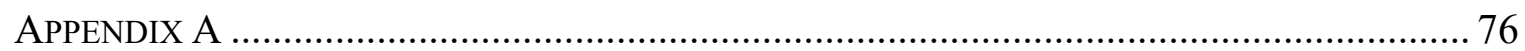

5 STUDY 3: A DIARY STUDY ON ANTICIPATED LEISURE TIME, MORNING RECOVERY, AND EMPLOYEES' WORK ENGAGEMENT .................................... 78

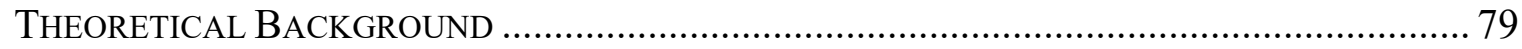

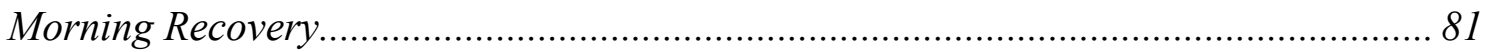

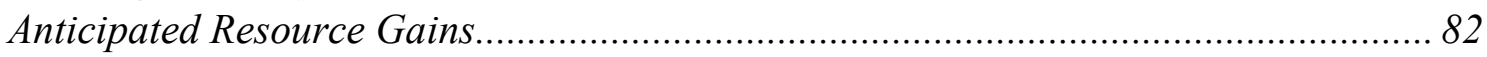

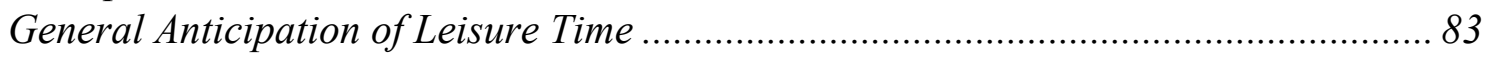

Pleasant Anticipation of a Planned Leisure Activity ..................................................... 83

Thoughts of a Planned Leisure Activity (ToPLA) as a Mediator ................................... 84

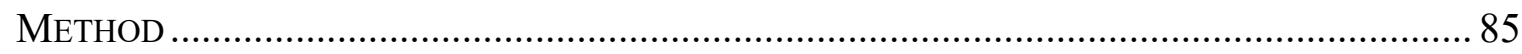

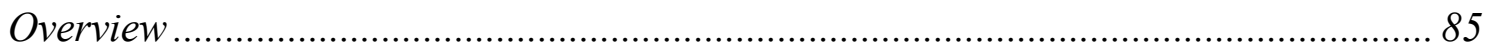

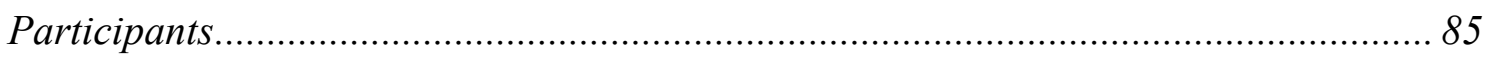

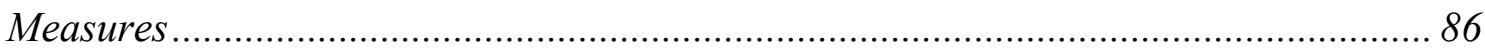




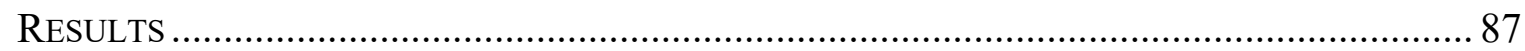

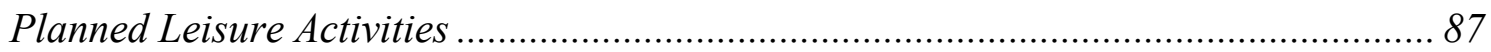

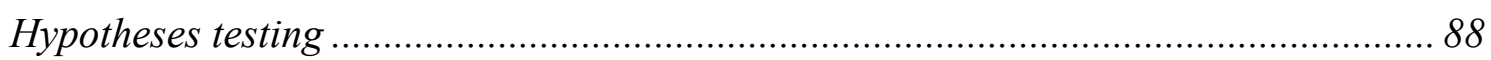

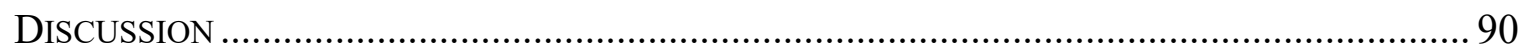

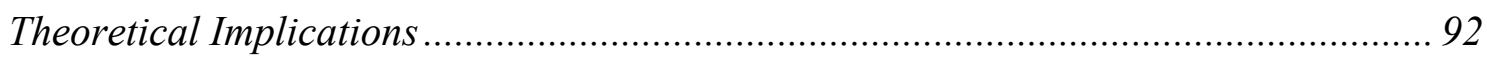

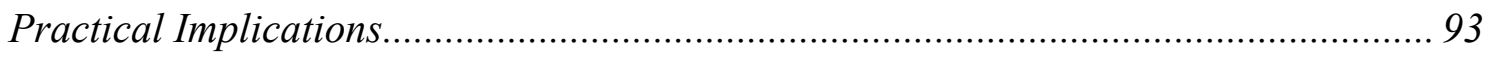

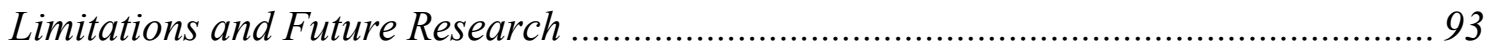

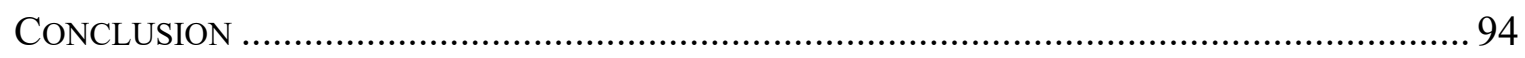

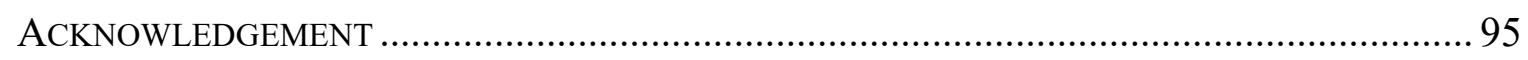

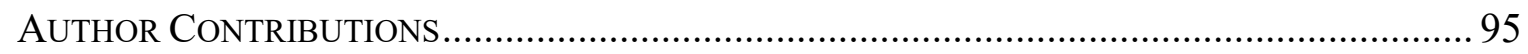

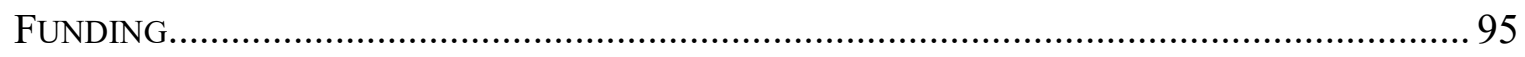

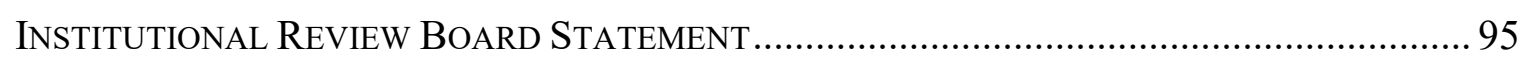

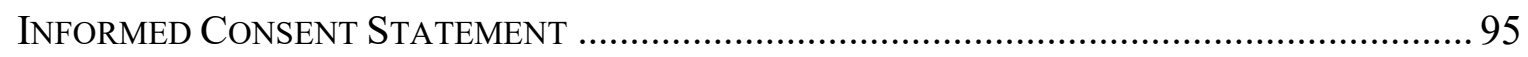

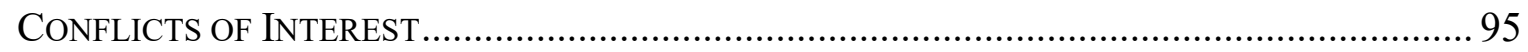

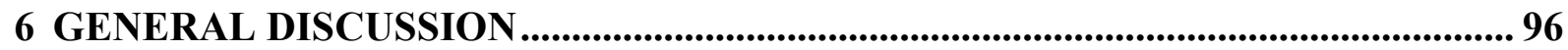

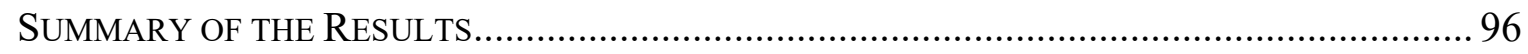

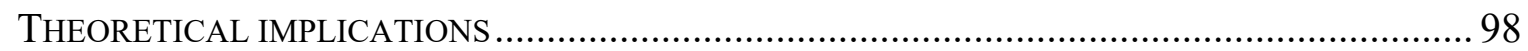

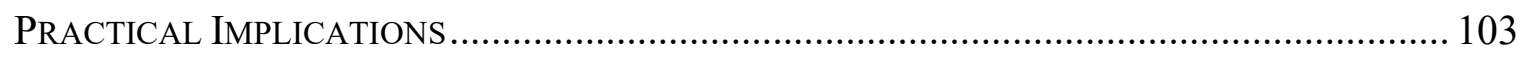

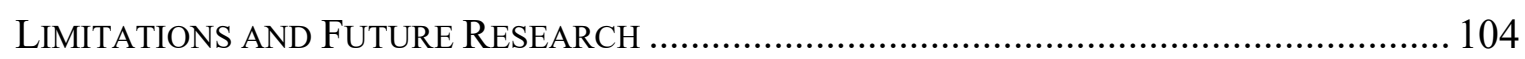

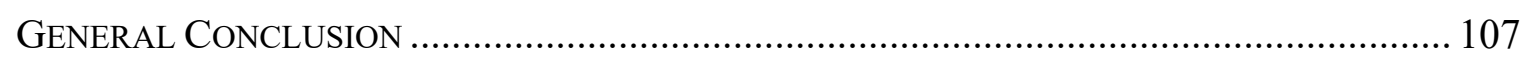

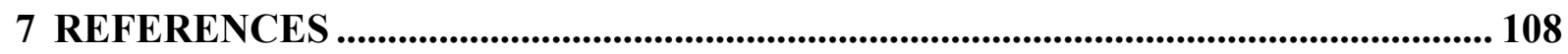




\section{GENERAL INTRODUCTION}

During the working day, employees engage in various demanding tasks, which drain their resources (Meijman \& Mulder, 1998; Trougakos \& Hideg, 2009). Exposure to these job demands is associated with psychological strain in the short and long run (for a meta-analysis, see Ford et al., 2014). However, research has demonstrated that recovery during leisure time reduces the impact of work demands and helps employees to stay healthy (for a meta-analysis, see Steed et al., 2019). Specifically, much recovery research has focused on leisure activities, which offer different opportunities for employees to recover from work stress (for reviews, see Demerouti et al., 2009; Fritz \& Demsky, 2019). Because leisure activities provide recovery experiences like relaxation or detachment from work, they help employees be more vigorous and less fatigued even when job demands are high (for a meta-analysis, see Bennett et al., 2018).

Recovery during leisure time is also beneficial for employees' work engagement (e.g., Garrick et al., 2014; Sonnentag, 2003; ten Brummelhuis \& Bakker, 2012b). Work engagement refers to a positive, motivational work-related state (Schaufeli et al., 2006b) and is relevant for employees and organisations (for a review, see Bailey et al., 2017): On the one hand, work engagement is positively related to employees' well-being, for instance, better health (for a meta-analysis, see Halbesleben, 2010b) and reduced burnout symptoms (for a meta-analysis, see Goering et al., 2017). On the other hand, work engagement is associated with employees' performance (for a meta-analysis, see Christian et al., 2011) and objective ratings of the organisation (e.g., financial metrics, Schneider et al., 2018).

In recovery research, the conservation of resources theory (COR theory; Hobfoll, 1989; Hobfoll et al., 2018) has often been applied to explain the association between recovery and work engagement (e.g., Bosch et al., 2018; Garrick et al., 2014; ten Brummelhuis \& Bakker, 2012b). According to COR theory (Hobfoll et al., 2018), individuals try to save existing resources and strive for new resources. Resources are broadly defined as everything that has value for individuals (e.g., energies such as recovery experiences, personal characteristics such as self-efficacy) and helps individuals reach their goals (Halbesleben et al., 2014). Several metaanalyses showed that resources are related to work engagement (Crawford et al., 2010; Halbesleben, 2010b; Lesener et al., 2019), and therefore, engaging in leisure activities to 
re(gain) resources in the evening helps employees to be more engaged the next day (e.g., Garrick et al., 2014; Sonnentag, 2003; ten Brummelhuis \& Bakker, 2012b).

Until now, recovery research has focused on resource gains because of past events (e.g., leisure activities, micro-breaks). However, research from various fields of psychology (e.g., cognitive psychology, social psychology, and work and organisational psychology) showed that upcoming events affect current feelings (Baumgartner et al., 2008; Carrera et al., 2012), thoughts (Aspinwall, 2005; D'Argembeau et al., 2011), and behaviour (Baumeister et al., 2007; Gollwitzer, 1996; Oettingen, 2012). Regarding work and organisational psychology, a study by Casper and Sonnentag (2020) demonstrated that when employees anticipate a high workload and feel worried about their next day's work in the evening, they start less vigorous into the next day. Furthermore, the anticipation of time pressure was associated with lower performance in several experiments (Leroy \& Glomb, 2018b). Thus, future events shape individuals' behaviour, and, according to some researchers (Baumeister et al., 2007; van Boven \& Ashworth, 2007), the future is even more important than the past for individuals' emotions. For instance, van Boven and Ashworth (2007) showed that individuals tend to report more intensive emotions for anticipated than for past events. Thus, it seems reasonable that upcoming leisure activities and leisure time may also relate to employees' feelings, thoughts, and behaviour at work.

Because leisure time and leisure activities offer opportunities for employees to gain new resources (for a meta-analysis, see Steed et al., 2019), I hypothesize that leisure time and leisure activities indicate upcoming resource gains. The principle of anticipation is part of COR theory (Hobfoll, 1989; Hobfoll et al., 2018), and past, as well as anticipated resource gains and losses, are essential for employees' resource management (Gorgievski \& Hobfoll, 2008a; Hobfoll et al., 2003). For instance, the anticipation of a resource loss in an experimental setting was associated with lower performance (Niessen \& Jimmieson, 2016). Furthermore, research has demonstrated that anticipating decreased workload (i.e., time to regain resources) helps employees deal with the current workload without experiencing more strain (DiStaso \& Shoss, 2020). These findings point out that anticipated resource gains (e.g., upcoming leisure time and leisure activities) may be meaningful for employees' resource management at work. Because previous recovery research has focused on the relationship between past leisure activities and work engagement (Sonnentag, 2003; ten Brummelhuis \& Bakker, 2012b), and because work engagement is associated with resources (Crawford et al., 2010; Halbesleben, 2010b) and has several benefits for employees and organisations (for a review, see Bailey et al., 2017), it is the 
focal outcome variable in this dissertation. Thus, the three studies included in this dissertation investigate the relationship between the anticipation of leisure time and work engagement

\section{Aims and Outline of the Dissertation}

The current research on the anticipation of leisure time contains three studies. Each study introduces a new construct to investigate the anticipation of leisure time: pleasant anticipation of a planned leisure activity is presented in Study 1, leisure thoughts are examined in Study 2, and general anticipation of leisure time is investigated in Study 3. By introducing these constructs, the dissertation adds to the literature in several ways and pursues three overarching research goals, which are explained in more detail in the following.

\section{First Research Goal: The Relationship between the Anticipation of Leisure Time and Work Engagement}

This dissertation is the first research that sheds light on upcoming leisure time and leisure activities as anticipated resource gains, and therefore, this research adds to COR theory (Hobfoll, 1989; Hobfoll et al., 2018) and recovery research (e.g., Bliese et al., 2017). While COR theory (Hobfoll, 1989; Hobfoll et al., 2018) has often been applied to recovery research, few researchers have used the idea of anticipated resource gains (for an exception, see Harris et al., 2007), although expectations about the future have consequences for current feelings, thoughts, and behaviour (Aspinwall, 2005; Baumeister et al., 2007; Baumgartner et al., 2008; Carrera et al., 2012; D'Argembeau et al., 2011; Oettingen, 2012). Because past leisure time and leisure activities are beneficial for employees, the findings of the following studies may reveal how employees could also benefit from upcoming leisure time and leisure activities. Furthermore, regarding work engagement, Hu et al. (2017) proposed that "[...] engagement is the result of the process of real or anticipated resource gain that enhances one's energy." (p. 642). However, this assumption has not been studied yet, and this dissertation may provide the first hint of whether anticipated resource gains are related to work engagement.

To investigate anticipated resource gains, I refer to pleasant anticipation of a planned leisure activity. Pleasant anticipation is a positive, affective reaction because of an upcoming event, and individuals only experience pleasant anticipation for positive events with a high probability of occurrence (Baumgartner et al., 2008). Researchers have used different short definitions for (pleasant) anticipation (e.g., Kong et al., 2011a; Sonnentag, Mojza, et al., 2008; Syrek, Weigelt, et al., 2018), focusing on one aspect of pleasant anticipation (e.g., the affective reaction or the upcoming event), yet not on the entire construct. Therefore, based on a review 
of these previous definitions, pleasant anticipation is defined in detail in Study 1, adding to research on future-oriented emotions (e.g., Baumgartner et al., 2008; Graham et al., 2019).

Furthermore, because a growing amount of research has concentrated on anticipation in the working context, e.g., the anticipation of time pressure (Leroy \& Glomb, 2018b), workload (Casper et al., 2017; DiStaso \& Shoss, 2020), or unpleasant tasks (Weigelt et al., 2019b), research on anticipation is extended by considering the anticipation of leisure time. Thus, this dissertation aims at investigating pleasant anticipation and thereby stimulating future research on this concept. I hypothesize that pleasant anticipation of a planned leisure activity indicates an upcoming resource gain and, based on COR theory (Hobfoll, 1989; Hobfoll et al., 2018), is positively associated with work engagement.

In addition, employees' recovery-related self-efficacy (RRSE; Sonnentag \& Kruel, 2006) is supposed to moderate the positive relationship between pleasant anticipation of a planned leisure activity and work engagement. RRSE indicates employees' beliefs about their ability to recover from work stress, and, like general self-efficacy (Bandura, 1995), RRSE is based on past experience. Therefore, the anticipated resource gain may increase when employees had positive experiences in their past leisure time. Thus, this research sheds light on interindividual differences, which may change the relationship between pleasant anticipation of a planned leisure activity and work engagement, and adds to the literature on RRSE (Hahn et al., 2011; H. I. Park \& Lee, 2015; Y. Park \& Sprung, 2015). In Study 1, the relationship between pleasant anticipation of a planned leisure activity, RRSE, and work engagement is investigated. To consider within- and between-person differences, Study 1a focuses on experiences within a single working day, while Study $1 \mathrm{~b}$ contains five consecutive working days within one week.

In sum, the focus of Study 1 is on pleasant anticipation of a planned leisure activity. However, because pleasant anticipation is limited to one specific, planned leisure activity, general anticipation of leisure time is introduced in Study 3. General anticipation of leisure time is an additional indicator of an anticipated resource gain, referring to the entire upcoming leisure time after the working day. While pleasant anticipation of a planned leisure activity is an affective reaction because of an upcoming leisure activity, general anticipation of leisure time is defined as a cognitive evaluation of the entire leisure time. Cognitive evaluation means employees have to retrieve information about leisure activities and other events in their upcoming leisure time and combine them into one rating. By introducing general anticipation of leisure time, the upcoming leisure time is assessed more generally to complement the research on the anticipation of leisure time. 


\section{Second Research Goal: Antecedents and Consequences of Leisure Thoughts}

To further investigate the associations between upcoming leisure time, leisure activities, and work engagement, I introduce employees' leisure thoughts in Study 2. During the working day, employees think about their working tasks (i.e., on-task thoughts) and report off-task thoughts, such as daydreams and wandering thoughts (Beal et al., 2005; Dane, 2018). Some of these offtask thoughts (e.g., Beal et al., 2005; Kanfer \& Ackerman, 1989; Gardner et al., 1989) refer to upcoming leisure time (Barsics et al., 2016). However, previous research did neither focus on the consequences nor the antecedents of leisure thoughts. Hence, I investigate antecedents and consequences of leisure thoughts and define two different types of leisure thoughts, namely thoughts of leisure time (ToLT) and thoughts of a planned leisure activity (ToPLA). While ToLT include all thoughts about the upcoming leisure time, ToPLA are restricted to thoughts about a specific, planned leisure activity. Therefore, ToPLA are in line with the conceptualisation of pleasant anticipation of a planned leisure activity. When employees experience high pleasant anticipation of a planned leisure activity (e.g., feeling excited because of the leisure activity), they may think more often about that activity. Thus, pleasant anticipation of a planned leisure activity is supposed to relate positively to ToPLA, yet not to ToLT. Furthermore, I posit that both types of leisure thoughts occur more frequently at the beginning and the end of the working day when work and leisure time are closer in time. Thus, this research adds to research on future-oriented thoughts (e.g., Aspinwall, 2005; D'Argembeau et al., 2011; Miranda et al., 2017) by introducing leisure thoughts as a special type of futureoriented thoughts and investigating the time of the day and pleasant anticipation as antecedents of leisure thoughts.

Regarding the consequences of leisure thoughts, both types of leisure thoughts are offtask thoughts distracting individuals from the task at hand (for a meta-analysis, see Randall et al., 2014), and therefore, I expect that they are negatively related to work engagement within one hour and across a working day. However, Dane (2018) proposed that off-task thoughts also have positive consequences for employees' performance, for instance, when these thoughts help employees plan their work tasks or remind them of their goals. Because ToPLA may remind employees of an upcoming resource gain, they may have positive consequences. Thus, when a leisure activity is positive (i.e., positive leisure plan valence), ToPLA may enhance employees' work engagement in the subsequent hour, although ToPLA are a type of off-task thoughts. Onand off-task thoughts have been discussed to explain employees' performance in previous research (Beal et al., 2005; Dane, 2018; Kanfer \& Ackerman, 1989). However, until now, different types of off-task thoughts have not been discussed in detail. By introducing and 
focusing on leisure thoughts, the previous research is extended to special types of off-task thoughts, and differences between these types are considered to explain differences in work engagement. In sum, the focus of Study 2 is on leisure thoughts, and Study 2 contains up to twelve hourly measurement points on one working day.

\section{Third Research Goal: Extending and Replicating the Results from Study 1 and Study 2}

Based on Study 1 and Study 2, two different mechanisms explaining the relationship between pleasant anticipation of a planned leisure activity and work engagement are discussed in Study 3: On the one hand, pleasant anticipation is supposed to indicate an upcoming resource gain, and therefore, may directly, positively relate to work engagement. On the other hand, because pleasant anticipation may be positively related to ToPLA, which drives the attention away from the task at hand, the relationship between pleasant anticipation and work engagement may be negative and mediated by ToPLA.

Furthermore, to demonstrate that the anticipation of leisure time makes a unique contribution to explaining work engagement, it is necessary to compare the anticipation of leisure time (i.e., upcoming resource gains) with past leisure time (i.e., gained resources). Hence, I ascertain whether morning recovery, one indicator of gained resources because of past leisure time, and anticipated resource gains because of upcoming leisure time and leisure activities are simultaneously related to work engagement. The results may help to understand the role of past and anticipated resource gains for employees' work engagement and add to COR theory (Hobfoll, 1989; Hobfoll et al., 2018) by considering past and anticipated resource gains and losses simultaneously. In sum, Study 3 aims at replicating and extending the previous studies of this dissertation and consists of an online daily diary study across one working week. Before the three studies are presented, the theoretical framework for all three studies is discussed. 


\section{2}

\section{THEORETICAL FRAMEWORK}

In all three studies, I draw on the conservation of resources (COR) theory (Hobfoll, 1989; Hobfoll et al., 2018) to explain the role of anticipated resource gains for employees' work engagement. Because the theoretical background is consistent across all three studies, an overview of COR theory (Hobfoll, 1989; Hobfoll et al., 2018) and the mechanism that may connect anticipated resource gains and work engagement is provided first. Afterwards, as work engagement is considered as an outcome variable in all studies, a brief introduction to the construct of work engagement is presented. Finally, the focal constructs of this dissertation (pleasant anticipation of a planned leisure activity, leisure thoughts, general anticipation of leisure time) are introduced, and their relevance for research on anticipation of leisure time is highlighted.

\section{Conservation of Resources (COR) Theory}

COR theory was developed as a new model of stress by Hobfoll (1989). The theory is based on the tenet that individuals try to obtain and protect their resources and are motivated to gain new resources (Hobfoll, 1989). According to COR theory (Hobfoll, 1989), individuals experience stress when they lose resources, invest resources without a consecutive resource gain, or feel threatened by a resource loss (i.e., anticipate a resource loss). Hobfoll (1989) defined resources as objects, conditions, personal characteristics, and energies, which have an instrumental or symbolic value for individuals. Instrumental value refers to the idea that individuals can use resources as tools to attain new and other resources, and symbolic value means that resources help individuals define their identity. Because, according to this broad definition, nearly everything positive could be regarded as a resource (Gorgievski et al., 2011; Thompson \& Cooper, 2001), the definition from Halbesleben et al. (2014) is used in this dissertation: resources are everything that helps individuals achieving their goals.

Energy resources are an essential resource type applied in recovery research (Halbesleben et al., 2014). Energy resources were initially defined as resources that individuals use to gain other resources, and examples of energy resources are money and time (Hobfoll, 1989). Because leisure time provides employees with additional time, recovery research suggested that leisure time and recovery experiences are also energy resources (Halbesleben et al., 2014). For 
instance, employees can use their leisure time to master new challenges improving their selfefficacy (a personal characteristic), or to make new contacts providing social support (a socalled condition resource). These examples show that different resources could be (re)gained during leisure time, and therefore, upcoming leisure time may indicate upcoming resource gains.

To better understand the interactions between resource investment and past as well as anticipated resource losses and gains, several extensions were made to COR theory (Halbesleben et al., 2014). A review by Hobfoll et al. (2018) summarized four principles of COR theory: principle 1 describes that resource losses are more salient than resource gains. Furthermore, individuals must invest resources to gain new resources (principle 2), and opportunities to gain resources become more salient when individuals have few resources (principle 3). Finally, principle 4 describes that individuals focus on their remaining resources and stop investing resources when their available resources are exhausted.

Employees' available resources may become depleted during the working day, as they have to deal with many demanding situations (Meijman \& Mulder, 1998; Trougakos \& Hideg, 2009). Accordingly, some employees may reach a point where they have few resources left, and therefore, they stop investing and start saving resources. I define this turning point of resource investment as the lower resource limit. When employees reach their lower resource limit, they enter a defensive mode of behaviour (e.g., invest less effort into work). However, this lower resource limit may decrease when employees anticipate their leisure time and expect an upcoming resource gain. Because they expect to (re)gain resources soon in their leisure time, they do not need to save many resources. It is important to note that anticipated resource gains are not added to employees' available resources but decrease their lower resource limit. Thus, while a recovered employee has more available resources, an employee who anticipates a resource gain has a reduced lower resource limit. However, both employees can invest more resources into their work engagement before reaching their lower resource limit and saving resources. Because work engagement fluctuates between days (Sonnentag et al., 2010) and hours (Syrek, Kühnel, et al., 2018) and these changes are sensitive to employees resource management, a decrease in employees' lower resource limit because of an upcoming resource gain may also relate to changes in work engagement. 


\section{Work Engagement}

The concept of work engagement was initially named job engagement and introduced by Maslach et al. (2001) to describe the positive antipode of burnout ${ }^{1}$. In contrast to burnout, which contains exhaustion, depersonalisation, and inefficiency, job engagement contains energy, involvement, and efficacy (Maslach et al., 2001). This conceptualisation of job engagement was further extended and defined as work engagement, describing a positive, motivational workrelated state characterised by vigour, dedication, and absorption (Schaufeli, Salanova, et al., 2002). To assess work engagement, the Utrecht Work Engagement Scale (UWES) was developed and validated (Schaufeli, Martínez, et al., 2002; Schaufeli, Salanova, et al., 2002). The validation studies demonstrated that burnout and work engagement load on different factors, and several subsequent studies provided evidence that burnout and work engagement are distinct states (for a meta-analysis, see Goering et al., 2017). Work engagement has soon become a well-investigated construct, and a large number of meta-analyses exist examining antecedents, consequences, and the nomological network of work engagement (e.g., Crawford et al., 2010; Goering et al., 2017; Lesener et al., 2019).

Important antecedents for work engagement are resources, for instance, feedback, social support, optimism, self-efficacy (Bakker \& Demerouti, 2017), and many meta-analyses showed that various resources are positively associated with work engagement (Crawford et al., 2010; Halbesleben, 2010b; Lesener et al., 2019; Nahrgang et al., 2011). Furthermore, job demands (e.g., workload, role conflict) are related to employees' work engagement (Crawford et al., 2010; Halbesleben, 2010b); however, the direction of this relationship is ambiguous: While hindrance demands are negatively related to work engagement, challenging demands are positively or negatively associated with work engagement (for a review, see Mazzola \& Disselhorst, 2019).

Regarding the consequences of work engagement, work engagement is crucial for employees and organisations. For instance, work engagement is positively related to health and negatively related to burnout (for a meta-analysis, see Goering et al., 2017). In addition, work engagement is associated with life satisfaction (Hakanen \& Schaufeli, 2012). Thus, high work engagement is beneficial for employees. Work engagement is also related to job performance (for a meta-analysis, see Christian et al., 2011) and financial metrics (Schneider et al., 2018). Furthermore, engaged employees report higher job satisfaction and organisational commitment,

\footnotetext{
${ }^{1}$ Kahn (1990) earlier used the term personal engagement to describe that employees bring in their personal self (physically, cognitively, and emotionally) into work.
} 
and less turnover intention (for a meta-analysis, see Goering et al., 2017). Thus, work engagement is also essential for organisations. In summary, work engagement is an extremely popular construct (Byrne et al., 2016), and a considerable amount of research investigated antecedents and consequences of employees' work engagement and related constructs (for a review, see Bailey et al., 2017) ${ }^{2}$.

Work engagement was investigated in various studies, e.g., studies on the relationship between work engagement and leadership (Breevaart et al., 2014; e.g., Tims et al., 2011), innovative behaviour (Orth \& Volmer, 2017), smartphone use during work (Syrek, Kühnel, et al., 2018), or sleep quality (Kühnel et al., 2017). Furthermore, much research has considered the association between work engagement, leisure time, leisure activities, and recovery (e.g., Breevaart et al., 2020; Garrick et al., 2014; Sonnentag, 2003; Sonnentag, Mojza, et al., 2008; ten Brummelhuis \& Bakker, 2012b). For this reason, and because of the importance of work engagement for employees and organisations, work engagement is considered an outcome variable.

\section{Leisure Time and Leisure Activities}

Before investigating the anticipating of leisure time, it is first necessary to define leisure time. However, many different definitions of leisure time exist (Kabanoff, 1980; Stebbins, 2018; Voss, 1967); for instance, leisure time is defined as the time individuals do not spend working to earn money (Aron, 1962, as cited in Voss, 1967). Another definition of leisure time refers to time individuals can spend on their own choice because they are free of other obligations (Voss, 1967). In addition, Parker and Smith (1976) differentiated the time not belonging to the regular working hours into work-related time (e.g., commute to work, prepare work at home), existence time (e.g., eating, sleeping), semi-leisure time (non-work obligations, e.g., walking the dog, gardening), and leisure time (i.e., time completely free to spend on own choice). Defining leisure time as time free of other obligations shows that the definition reflects employees' subjective evaluation of what leisure time means for them (Geurts \& Demerouti, 2003; Kabanoff, 1980). For instance, while gardening may be an obligation for some individuals, other persons may enjoy gardening and spend time gardening on their own choice to relax and detach from work. The different evaluations of gardening may similarly apply to other leisure activities, such as cooking, shopping, repairing a car. Therefore, I refer to the broad definition

\footnotetext{
${ }^{2}$ Related constructs are, for instance, employee engagement and personal engagement Shuck (2011). Differences between the constructs are small (e.g., employee engagement refers not only to work, but also to the team and the organization), and the different constructs are often mixed in meta-analysis e.g., in the meta-analysis by Lesener et al. (2019).
} 
of leisure time as the non-work time before and after the working day (Aron, 1962, as cited in Voss, 1967).

During their leisure time, employees can engage in different activities. In recovery research, these activities are commonly classified as leisure activities or high-duty activities (Sonnentag, 2001; ten Brummelhuis \& Bakker, 2012b). Regarding leisure activities, research further differentiated between social activities (e.g., meeting friends), physical activities (e.g., going to the gym), and low-effort activities (e.g., reading a book, watching TV). Furthermore, three types of high-duty activities exist (Sonnentag, 2001; ten Brummelhuis \& Bakker, 2012b): work-related activities, childcare activities, and household activities (e.g., cooking, gardening).

In general, leisure activities are supposed to be beneficial for employees' recovery, while high-duty activities may hinder employees' recovery (Sonnentag, 2001; ten Brummelhuis \& Bakker, 2012b). However, the findings for this classification are not consistent across studies (for a review, see Sonnentag et al., 2017), and meta-analytical results are mixed (Steed et al., 2019). For instance, leisure activities are associated with higher psychological well-being but not with sleep quantity or quality (Steed et al., 2019). One explanation may be that some highduty activities may also be regarded as leisure activities. For instance, because gardening requires physical effort and some employees may enjoy gardening, it may be a leisure or highduty activity. Thus, I refer to leisure activities as all activities that employees may engage in during their leisure time, which concurs with the broad definition of leisure time.

Based on these definitions, the constructs of pleasant anticipation of a planned leisure activity, leisure thoughts, and general anticipation of leisure time are developed to investigate the relationship between the anticipation of leisure time and work engagement.

\section{Pleasant Anticipation of a Planned Leisure Activity}

In Study 1, pleasant anticipation of a planned leisure activity is introduced as an indicator of an anticipated resource gain from various leisure activities. Different leisure activities are related to different recovery experiences, such as relaxation and detachment from work (Mojza et al., 2010; ten Brummelhuis \& Bakker, 2012b), and research has demonstrated that these different recovery experiences address different resources (Ragsdale et al., 2011; Ragsdale \& Beehr, 2016). For instance, in a study by Ragsdale and Behr (2016), spending time on social activities helps employees experience a feeling of control over leisure time (a recovery experience), and in turn, enhances their self-regulatory capacity (i.e., a resource). Furthermore, different recovery profiles have been found (Chawla et al., 2020; de Bloom et al., 2018), and research showed that employees enjoy different leisure activities (Oerlemans et al., 2014; van 
Hooff \& Pater, 2017). Hence, to study the anticipation of leisure activities, developing a single indicator covering different resource gains of various upcoming leisure activities is necessary. Therefore, based on past work on anticipation (Baumgartner et al., 2008; Loewenstein, 1987), pleasant anticipation of a planned leisure activity is introduced.

I define pleasant anticipation as a positive affective reaction because of an upcoming event with a high probability of occurrence. This conceptualisation is based on Baumgartner et al. (2008), who used the term pleasant anticipation as a translation of the German word "Vorfreude" or the Dutch word "vor-preet". However, different conceptualisations of (pleasant) anticipation exist, all with different names and foci. For instance, in an early work, Jevons (1905, as cited in Loewenstein, 1987, p. 667) used the term "anticipal [sic.] pleasure" to refer to pleasurable feelings that are caused by future events. Furthermore, Kong et al. (2011a) used anticipated happiness to describe expected happiness when a future event occurs. Moreover, the term wanting and desire was used to express the feeling when the probability of occurrence for a future event is high (Bruininks \& Malle, 2005). These different definitions are summarized in Table 1 of Study 1 (p. 30 of this dissertation). In sum, (pleasant) anticipation often describes either affective reactions or characteristics of the future event. In contrast, I argue that pleasant anticipation combines an affective reaction with characteristics of the future event.

I assume that pleasant anticipation contains four unique characteristics which differentiate pleasant anticipation from other affective reactions. First, pleasant anticipation refers to a specific, positive event, and individuals are aware of that event. Thus, individuals know why they experience pleasant anticipation. Conversely, individuals can experience joy or happiness independently of a specific event. Second, pleasant anticipation can only be experienced for future events, yet it cannot be experienced when individuals remember past events or think of their present situation. Third, to experience pleasant anticipation, the occurrence of the future event has to be certain. In contrast, experienced uncertainty of future events evokes other affective reactions such as hope (Bruininks \& Malle, 2005; Roseman, 1996). Finally, pleasant anticipation can be a combination of different affective reactions, and individuals can feel excited, happy, and/or enthusiastic simultaneously in anticipation of a future event.

One advantage of using pleasant anticipation to refer to future events (e.g., planned leisure activities) is that pleasant anticipation combines an affective reaction at present with characteristics of the future event (expectation of the outcome, probability of occurrence). To experience pleasant anticipation of a planned leisure activity, employees must have a proper image of the upcoming leisure activity and know the value and the probability of occurrence for that leisure activity. Therefore, pleasant anticipation of a planned leisure activity indicates 
the ideocratic value of the future event and represents an individual affective reaction to the anticipated resource gain. This affective reaction can be compared between employees even if they anticipate different leisure activities and types of resources. Furthermore, different leisure activities could be compared between different days within the same employee. Hence, pleasant anticipation of a planned leisure activity is used in all three studies as an indicator of an anticipated resource gain and is assumed to be positively related to employees' work engagement (Study 1-3) and leisure thoughts (Study 2, Study 3).

\section{Leisure Thoughts}

Leisure thoughts are introduced as a new construct in Study 2 and defined as all thoughts that refer to today's upcoming leisure time. Because thinking about leisure time is not part of employees' regular working tasks (i.e., on-task thoughts), leisure thoughts are a type of off-task thoughts (Beal et al., 2005; Gardner et al., 1989; Kanfer \& Ackerman, 1989). While off-task thoughts distract individuals from their task at hand and reduce performance, on-task thoughts are related to performance (for a meta-analysis, see Randall et al., 2014). However, off-task thoughts refer to a variety of different contents located in the past, present, and future (Martin \& Tesser, 1996; Mooneyham \& Schooler, 2013; Smallwood \& Schooler, 2015) ${ }^{3}$, and therefore, the relationship between off-task thoughts and employees' performances may depend on the content of the off-task thoughts.

Until now, no systematic research on leisure thoughts exists, but research on futureoriented thoughts has considered leisure as one content of future-oriented thoughts (D'Argembeau et al., 2011). These future-oriented thoughts are triggered for different reasons (e.g., unattained goals, negative mood; Mrazek et al., 2011; Smallwood \& Schooler, 2015; Watkins, 2008) and, like other off-task thoughts, may have positive or negative consequences for individuals (Dane, 2018; Smallwood \& Schooler, 2013; Watkins, 2008). On average, individuals think about the future 59 times per day, resulting in about one thought every 16 minutes (D'Argembeau et al., 2011). Furthermore, research has demonstrated that most futureoriented thoughts (35\%) are about leisure time (D'Argembeau, 2018) and 25\% occur at work (Barsics et al., 2016). Thus, there is initial evidence that employees experience leisure thoughts; however, the consequences could be positive (e.g., remind employees of an upcoming resource gain) or negative (distract employees from the task at hand).

\footnotetext{
${ }^{3}$ The term off-task thoughts refers to mind-wandering, spontaneous thoughts, daydreaming and other thoughts that do not refer to the task at hand for a review, see Marchetti et al. (2016)
} 
To further investigate leisure thoughts, two types of leisure thoughts are differentiated. The first type is defined as thoughts of leisure time (ToLT) and refers to all thoughts about the upcoming leisure time. For instance, employees may think of what they may eat after work or whether they have to do some errands. Thus, ToLT represent off-task thoughts about the entire leisure time. The second type is defined as thoughts of a planned leisure activity (ToPLA) and refers to thoughts about one specific planned leisure activity. For instance, employees think about a planned meeting with friends after the working day or about the physical activity they want to engage in after work. It is important to note that ToPLA are part of ToLT, and when more than one planned leisure activity exists, separate sets of ToPLA for the different activities may also exist. The relationship between pleasant anticipation, ToLT and ToPLA, and work engagement is examined in Study 2 and Study 3. Moreover, in Study 3, ToPLA were investigated as a mediator between pleasant anticipation and work engagement.

\section{General Anticipation of Leisure Time}

Because pleasant anticipation of a planned leisure activity only refers to one specific, planned leisure activity, other events and activities during leisure time are neglected. Hence, general anticipation of leisure time is introduced in Study 3 and defined as a cognitive, overall evaluation of the entire upcoming leisure time after one working day. Like other overall evaluations (e.g., evaluations of names, Gebauer et al., 2008; leader satisfaction, van Quaquebeke et al., 2010; job satisfaction, Wanous et al., 1997), general anticipation is not tied to a specific aspect of the upcoming leisure time (e.g., a planned leisure activity). Thus, general anticipation of leisure time takes all parts of the upcoming leisure time into account. General anticipation and pleasant anticipation are supposed to indicate a resource gain in the upcoming leisure time; however, they address different parts of leisure time. While pleasant anticipation only refers to a planned leisure activity, general anticipation of leisure time goes beyond this activity and reflects a daily evaluation of the entire leisure time after the working day.

Furthermore, employees may consider present circumstances at work when evaluating their general anticipation of leisure time. For instance, when employees experience time pressure at work, they may anticipate their upcoming leisure time to a higher degree than when time pressure is low. The use of the present work situation as an anchor to evaluate the upcoming resource gain concurs with COR theory's third Principle (Hobfoll et al., 2018), which describes that potential resource gains (e.g., anticipated leisure time) become more salient when employees lose resources (e.g., because of time pressure at work). Thus, employees contrast their present work situation with their upcoming leisure time to evaluate the value of their 
upcoming leisure time. Because employees need to retrieve and combine information for this evaluation process, general anticipation of leisure time is named a cognitive evaluation. Moreover, in contrast to the affective reaction of pleasant anticipation, general anticipation should not evoke a strong affective reaction. Because both constructs may be crucial to understand the relationship between anticipated leisure time and work engagement, they are simultaneously investigated in Study 3. 


\title{
3
}

\section{STUDY 1: PLEASANT ANTICIPATION OF LEISURE TIME AND WORK ENGAGEMENT: FINDINGS FROM TWO STUDIES}

\author{
Sebastian Seibel ${ }^{a b}$, Judith Volmer ${ }^{a}$, and Antje Schmitt ${ }^{\mathrm{c}}$ \\ ${ }^{a}$ Work and Organizational Psychology Group, Otto-Friedrich University of Bamberg, \\ Bamberg, Germany; \\ ${ }^{b}$ Work and Organizational Psychology Group, University of Wuerzburg, Wuerzburg, \\ Germany; \\ ${ }^{c}$ Department of Psychology, University of Groningen, Groningen, the Netherlands
}

This is an original manuscript of an article that has been revised and resubmitted to Leisure Science (Tylor \& Francis) and is currently under review.

\begin{abstract}
After a working day, leisure activities offer employees the opportunity to regain resources. Hence, employees may look forward to planned leisure activities. Building on the conservation of resources (COR) theory, we assumed that employees' pleasant anticipation of a planned leisure activity indicates an upcoming resource gain and is therefore positively related to employees' work engagement within and between persons. In addition, we examined the moderating role of recovery-related self-efficacy (RRSE) in this relationship. In Study 1a, employees completed three questionnaires (morning, noon, afternoon) on one working day, and we expanded the design to a one-week diary in Study 1b. Findings from (hierarchical) linear modelling supported the positive relationship between pleasant anticipation and work engagement between persons but not within persons. Furthermore, RRSE did not moderate the relationship between pleasant anticipation and work engagement. Overall, our study demonstrated that employees differ in their pleasant anticipation, partly explaining differences in work engagement.
\end{abstract}

Keywords: pleasant anticipation; work engagement; recovery-related self-efficacy; conservation of resources theory; leisure time; work motivation 


\section{Theoretical background}

During leisure time, employees can engage in various leisure activities, offering them the opportunity to recover and gain new resources (e.g., Oerlemans et al., 2014; Sonnentag, 2001; ten Brummelhuis \& Bakker, 2012b). Thus, leisure time is essential for employees' recovery from work-related stress (for a review, see Sonnentag et al., 2017). Hobfoll's (1989) conservation of resources (COR) theory has often been applied in recovery research and addresses employees' resource management (e.g., Binnewies et al., 2009; Fritz \& Sonnentag, 2005; Volman et al., 2013). For instance, research grounded on COR theory has shown that recovered employees report higher task performance (Binnewies et al., 2009) and work engagement (Sonnentag, 2003). While COR theory considers past and future resource gains and losses (Gorgievski \& Hobfoll, 2008b; Hobfoll, 2001b), most research has focused on past leisure activities. For our study, we extend this view by exploring upcoming leisure activities as a potential source of resource gains.

Hobfoll (1989) initially defined resources as everything that has a value for individuals and named four categories of resources (objects, conditions, personal characteristics, and energies). Because researchers interpreted and used this broad definition in different ways, everything individuals regard as helpful to attain their goals could be defined as a resource (Halbesleben et al., 2014; van den Heuvel et al., 2010). Many studies have demonstrated that present resources are associated with positive outcomes for employees, such as employee wellbeing and performance (for a review, see Nielsen et al., 2017). Besides present resources, some initial evidence exists that anticipated resource gains and losses are meaningful for employees' resource management (DiStaso \& Shoss, 2020; Niessen \& Jimmieson, 2016). For instance, the threat of losing resources (i.e., anticipated resource loss) is related to lower task performance in an experimental setting (Niessen \& Jimmieson, 2016). Furthermore, research has shown that anticipating a decrease in workload (i.e., time to gain or replenish resources) helps employees experience less strain even when the present workload is high (DiStaso \& Shoss, 2020).

Following these findings, we propose that employees consider their upcoming leisure activities (an anticipated resource gain) when managing their resources at work. As an indicator of this anticipated resource gain, we refer to employees' pleasant anticipation of a planned leisure activity, a positive affective reaction because of a future event. We expect that employees with higher pleasant anticipation show higher work engagement, a positive, motivational, work-related state (Bakker et al., 2008), comprising vigour, absorption, and dedication (Schaufeli, Salanova, et al., 2002). Apart from specific planned leisure activities, 
employees' beliefs about their ability to recover during leisure time may also influence their resource management. These beliefs are defined as recovery-related self-efficacy (RRSE; Sonnentag \& Kruel, 2006), and because RRSE may ascertain which employees benefit most from high pleasant anticipation, RRSE is supposed to moderate the relationship between pleasant anticipation and work engagement.

Our research contributes to the literature in several ways. First, we extend previous research that focused on past leisure activities and work engagement (e.g., Sonnentag, 2003; ten Brummelhuis \& Bakker, 2012b) by flipping the coin and investigating pleasant anticipation of a planned leisure activity as an antecedent of work engagement. These previous findings referred to COR theory (Hobfoll, 1989; Hobfoll et al., 2018) and argued that leisure activities help employees to (re)gain resources. Because COR theory also includes anticipated resource gains and losses (Gorgievski \& Hobfoll, 2008b; Hobfoll, 2001b), we examine whether anticipated leisure activities also contribute to employees' work engagement. Furthermore, considering RRSE provides the opportunity to investigate interindividual differences in resource management and in the extent to which employees benefit from pleasant anticipation of a planned leisure activity.

Second, work engagement has several positive consequences for employees and organisations, such as high well-being and performance and less burnout and turnover intention (for a review, see Bailey et al., 2017). Therefore, it is crucial to identify new factors, such as pleasant anticipation of a planned leisure activity, which may positively relate to work engagement. Third, we add to earlier research on anticipation in the working context, for instance, research on the anticipation of workload (Casper \& Sonnentag, 2020), time pressure (Leroy \& Glomb, 2018a), and vacation time (Nawijn et al., 2013). Because this research focused on negative events (workload, time pressure) or infrequent events (vacations), we choose a more positive approach and study pleasant anticipation, which refers to a positive future event and may be experienced every day.

Finally, to examine whether the relationship between pleasant anticipation and work engagement varies or is equivalent across and within individuals (McCormick et al., 2018), we conducted two studies using different time perspectives and measurement points. Study 1a contained one single working day focusing on between-person differences in the relationship between pleasant anticipation work engagement. However, as employees may not plan the same leisure activity every day, the relationship between pleasant anticipation and work engagement may vary within the same employee. Consequently, Study $1 \mathrm{~b}$ covered five consecutive working 
days to investigate within-person variability in pleasant anticipation and between-person differences in average pleasant anticipation across the week.

\section{Definition of Pleasant Anticipation}

Pleasant anticipation describes a positive affective reaction experienced at present in anticipation of an upcoming future event individuals are looking forward to (Baumgartner et al., 2008). For instance, individuals may feel happy because they expect a nice evening with friends, and they may experience excitement when they have not seen their friends for a long time. Compared to other affective reactions, pleasant anticipation has four specific characteristics (Seibel et al., 2020). First, pleasant anticipation refers to a specific, positive event that individuals are aware of. In contrast, individuals can experience other affective reactions (e.g., joy) not tied to a specific event. Second, pleasant anticipation can only be experienced for future events, but not when individuals remember past events. Third, to experience pleasant anticipation, the occurrence of the future event has to be certain, whereas for other futureoriented affective reactions, such as hope, the uncertainty of the future event is pivotal (Bruininks \& Malle, 2005; Roseman, 1996). Finally, pleasant anticipation may contain a combination of different affective reactions; for instance, individuals feel excited, happy, and enthusiastic simultaneously in anticipation of a future event. Pleasant anticipation is not the only term used for affective reactions that refer to upcoming events (for an overview, cf. Table 1). However, in contrast to the other conceptualizations such as anticipated happiness (Kong et al., 2011a) or anticipated enthusiasm (Graham et al., 2019), pleasant anticipation combines the high probability of a positive future with different affective reactions to consider idiosyncratic experiences.

\section{Pleasant Anticipation of a Planned Leisure Activity and Work Engagement}

According to the COR theory (Hobfoll, 1989; Hobfoll et al., 2018), individuals try to maximize their resources and protect themselves from resource losses. During the working day, employees face different job stressors, which all can drain their resources (Meijman \& Mulder, 1998; Trougakos \& Hideg, 2009). When employees' resources fall below a specific limit, they focus on their remaining resources, stop, or hesitantly invest further resources, and enter a defensive mode of behaviour (Hobfoll et al., 2018). We define this limit as the lower resource limit, indicating the turning point of resource investment. However, the lower resource limit may decrease when employees anticipate a resource gain in their leisure time. Because they expect an opportunity to (re)gain resources soon, they can make riskier resource investments, 
Table 1

Overview of Pleasant Anticipation and Related Constructs

\begin{tabular}{|c|c|c|c|c|}
\hline Construct & Definition & $\begin{array}{l}\text { Affective } \\
\text { reaction }\end{array}$ & Future event & Reference \\
\hline $\begin{array}{r}\text { Anticipal } \\
\text { pleasure }\end{array}$ & $\begin{array}{l}\text { Pleasure because of a } \\
\text { positive evaluation of } \\
\text { a future outcome }\end{array}$ & Pleasure & Positive & $\begin{array}{l}\text { Jevons (1905) } \\
\text { as citied in } \\
\text { Loewenstein, } \\
1987\end{array}$ \\
\hline $\begin{array}{c}\text { Anticipated } \\
\text { happiness }\end{array}$ & $\begin{array}{l}\text { Happiness individuals } \\
\text { expect when a future } \\
\text { event will occur }\end{array}$ & Happiness & Not specified & $\begin{array}{l}\text { Kong et al., } \\
2011 \mathrm{a}\end{array}$ \\
\hline Anticipation & $\begin{array}{l}\text { Anticipation of } \\
\text { enjoying a future } \\
\text { event }\end{array}$ & Not specified & Enjoyable & $\begin{array}{l}\text { Sonnentag, } \\
\text { Mojza, et al., } \\
2008\end{array}$ \\
\hline $\begin{array}{r}\text { Anticipatory } \\
\text { enthusiasm }\end{array}$ & $\begin{array}{l}\text { Enjoyable emotion } \\
\text { because of a positive } \\
\text { appraisal of future } \\
\text { outcome }\end{array}$ & Not specified & Positive & $\begin{array}{l}\text { Graham et al., } \\
2019\end{array}$ \\
\hline $\begin{array}{l}\text { Literally pre- } \\
\text { joy translated } \\
\text { as Pleasant } \\
\text { Anticipation }^{1}\end{array}$ & $\begin{array}{l}\text { Joy about a future } \\
\text { event, which is not } \\
\text { hypothetic. }\end{array}$ & Joy & $\begin{array}{l}\text { Not } \\
\text { hypothetic }\end{array}$ & $\begin{array}{l}\text { Baumgartner et } \\
\text { al., } 2008\end{array}$ \\
\hline $\begin{array}{l}\text { Pleasant } \\
\text { anticipation }\end{array}$ & $\begin{array}{l}\text { Positive affective } \\
\text { reaction because of a } \\
\text { positive future event } \\
\text { with a high } \\
\text { probability of } \\
\text { occurrence }\end{array}$ & $\begin{array}{l}\text { Positive } \\
\text { (e.g., Joy, } \\
\text { happiness, } \\
\text { excitement) }\end{array}$ & $\begin{array}{l}\text { High } \\
\text { probability; } \\
\text { Positive }\end{array}$ & $\begin{array}{l}\text { Seibel et al., } \\
2020 \text { based } \\
\text { on the } \\
\text { translation of } \\
\text { Baumgartner } \\
\text { et al., } 2008\end{array}$ \\
\hline $\begin{array}{l}\text { Wanting and } \\
\text { desire }\end{array}$ & $\begin{array}{l}\text { Experienced when the } \\
\text { probability of } \\
\text { attaining a positive } \\
\text { future is high. }\end{array}$ & Not specified & $\begin{array}{l}\text { High } \\
\text { probability }\end{array}$ & $\begin{array}{l}\text { Bruininks \& } \\
\text { Malle, } 2005\end{array}$ \\
\hline
\end{tabular}

Note. " This joy about a future event is called 'Vorfreude' in German and 'voorpret' in Dutch (literally pre-joy), and it may be translated as pleasant anticipation." (Baumgartner et al., 2008, p. 695)

which is reflected by a decrease in their lower resource limit. Consequently, they can invest more resources into their present working tasks before reaching the lower resource limit.

During leisure time, employees can engage in various leisure activities to (re)gain resources (e.g., Oerlemans et al., 2014; Sonnentag, 2001; ten Brummelhuis \& Bakker, 2012b), and each individual prefers different activities (de Bloom et al., 2018) and experiences more or less pleasure during leisure activities (van Hooff \& Pater, 2017). Thus, the anticipated resource gain depends on an individual evaluation of the planned leisure activity. Accordingly, the same leisure activity could be beneficial to (re)gain resources for some employees but not for others. 
To cover the potential resource gains from different leisure activities, we refer to pleasant anticipation of a planned leisure activity.

For instance, when employees experience pleasant anticipation of a relaxing leisure activity, they may become more engaged in their work, as they expect to regain their energy resources during leisure time. In addition, pleasant anticipation of physical activities may boost employees' work engagement, as employees expect detachment and recovery during these activities. These examples show that pleasant anticipation neither depends on the type of planned leisure activity (e.g., social, physical) nor refers to a specific anticipated resource gain (e.g., energy, recovery). Thus, pleasant anticipation highlights the overall personal value of different anticipated leisure activities, and higher pleasant anticipation indicates a higher and more likely resource gain during leisure time.

We argue that an upcoming resource gain indicated by high pleasant anticipation decrease employees' lower resource limit. Because work engagement fluctuates between weeks (Petrou et al., 2017), days (e.g., Orth \& Volmer, 2017; Xanthopoulou et al., 2008), and even hours (Seibel et al., 2020; Syrek, Kühnel, et al., 2018), changes in work engagement may be sensitive to changes in employees' lower resource limit. Thus, we suggest that employees with higher pleasant anticipation report higher work engagement than employees with lower pleasant anticipation (i.e., between-person differences on the same day). Furthermore, we suggest that pleasant anticipation and work engagement are positively related within-persons indicating that employees show higher work engagement on days with higher pleasant anticipation than on days with lower pleasant anticipation. Finally, as resources accumulate over time (Hobfoll, 1989; Hobfoll et al., 2018), we argue that employees' average pleasant anticipation of a planned leisure activity is associated with work engagement (between persons across the week). These three assumptions follow the same theoretical argumentation, with the main difference being the level (between and within employees across the week) at which conclusions could be drawn. Overall, we posit the following:

Hypothesis 1: Pleasant anticipation of a planned leisure activity will positively relate to work engagement within and between persons (on one day/across the week).

\section{Recovery-Related Self-Efficacy (RRSE) as a Moderator}

RRSE is a specific type of self-efficacy that refers to employees' beliefs about their ability to recover during leisure time (Sonnentag \& Kruel, 2006). Employees with high RRSE trust in their ability to benefit from leisure time, even when they are tired, angry, or have numerous work to do (Sonnentag \& Kruel, 2006). Research on RRSE has demonstrated that RRSE buffers 
the association between negative affect in the evening and poor sleep quality and low morning recovery (Y. Park \& Kim, 2019). Furthermore, RRSE is supposed to reduce the relationship between poor sleep quality and fatigue (H. I. Park \& Lee, 2015). Thus, employees with high RRSE may use their leisure time more efficiently and have more specific knowledge on how to recover during leisure time (e.g., detach from work, Yang et al., 2020). Consequently, we argue that employees' RRSE is crucial when employees evaluate their anticipated resources from planned leisure activities.

RRSE emerges from employees' past experiences during their leisure time, and past experiences may change how individuals rate their future opportunities. Therefore, RRSE may interact with employees' expectations about their anticipated leisure time. For instance, employees may experience high pleasant anticipation of a planned leisure activity; thus, their lower resource limit decreases. In addition, when employees trust their ability to benefit from that planned leisure activity (high RRSE), their lower resource limits further decrease, which is reflected by a stronger association between pleasant anticipation and work engagement. In contrast, when RRSE is low, employees are sceptical about their ability to recover, indicated by a weaker relationship between pleasant anticipation and work engagement. Thus, while high RRSE may strengthen the relationship between pleasant anticipation of a planned leisure activity and work engagement, low RRSE may reduce this relationship. It is important to note that, as self-efficacy (e.g., RRSE) is context-specific (Bandura, 1977, 1994), RRSE should only reduce the lower resource limit combined with pleasant anticipation.

Regarding differences between persons on the same day, employees with higher pleasant anticipation and higher RRSE may report higher work engagement than employees with high pleasant anticipation and low RRSE. Thus, RRSE is supposed to moderate the relationship between pleasant anticipation and work engagement. Also, we argue that the within-person relationship varies as a function of employees' RRSE (cross-level), indicating that employees with higher RRSE may experience a higher chance in work engagement on days with high pleasant anticipation compared to days with low pleasant anticipation. However, as RRSE is context-specific, RRSE refers to a single leisure activity and could not interact with an average leisure activity rating across the week. Therefore, RRSE is not expected to moderate the relationship between employees' average pleasant anticipation (across the week) and work engagement. In sum, we hypothesise the following:

Hypothesis 2: RRSE will moderate the relationship between pleasant anticipation and employees' work engagement such that the relationship will be stronger for 
employees with high RRSE and weaker for employees with low RRSE (between persons on one day/within persons on different days).

\section{Study 1a: Method}

We conducted an online survey study over one working day. Participants chose Tuesday, Wednesday, or Thursday for their day of participation. We decided to exclude Mondays and Fridays because of their unique characteristics regarding the week's rhythm, e.g., lower mood on Mondays (Areni et al., 2011) and higher mood on Fridays (Stone et al., 2012). Moreover, leisure activities for Fridays may differ from those on Tuesday-Thursdays, as plans for Fridays may include activities that last for the entire weekend.

\section{Procedure}

We recruited employees from different organisations by posting flyers on social media websites and using personal contacts. Furthermore, psychology students helped with data collection as part of a research course and a master's thesis project. The participants must be over 18 years old and work full time on the selected working day. Participation in the online study was voluntary, and participants could get a feedback report on their recovery experiences (Sonnentag \& Fritz, 2007), including some advice on successful recovery during leisure time.

Participants first chose one day on which they liked to participate in the survey, and afterwards, they received a link to a presurvey, which was available until one day before the survey. On the selected day, participants completed three short online questionnaires during the working day: a morning questionnaire (available from 04:00-10:00), a noon questionnaire (available from 11:00-14:00), and an evening questionnaire (available from 15:30-20:00). Participants were instructed to respond to the evening questionnaire just before leaving work. At each measurement point, participants received reminder emails with personal links to the online survey.

\section{Participants}

In total, 190 participants agreed to participate, and 137 participants responded to at least two questionnaires (response rate: $72.11 \%$ ). Because we included only participants in the analyses who completed the presurvey and the three daily questionnaires (morning, noon, afternoon), the final sample contained 85 participants who completed all four questionnaires $(54.36 \%$ completion rate). The participants (55.00\% were females) were, on average, 37.54 years old $(S D=13.21)$. Most of them held a master's degree $(31.59 \%)$ or had finished a vocational education (31.76\%). Participants worked in different occupational sectors (e.g., 12.94\% 
information \& communication, $10.59 \%$ health and social work), and $30.59 \%$ held a leadership position.

\section{Measures}

Our study consisted of a pre-survey and three short, daily online questionnaires. Unless otherwise stated, participants answered all items on five-point Likert scales $(1=$ strongly disagree; 5 = strongly agree).

\section{Recovery-Related Self-Efficacy (RRSE; Presurvey).}

RRSE was assessed with six items from Sonnentag and Kruel (2006). A sample item was, "I feel confident to be able to recover during off-job time even when I am tired”. Cronbach's $\alpha$ was .83.

\section{Pleasant Anticipation of a Planned Leisure Activity (Noon Questionnaire).}

First, participants were instructed to think of a planned leisure activity for about one minute and to describe this activity with a few words. Examples were "do physical exercises," "relax on the couch," "play music in a band," and "read a book." Afterwards, we assessed pleasant anticipation of this planned leisure activity with four items used in a previous study on the anticipation of leisure time (Seibel et al., 2020). A sample item read, "I am looking forward to my leisure activity." Cronbach's alpha was .94.

\section{Work Engagement (Afternoon Questionnaire).}

We used the short version of the Utrecht work engagement scale (UWES-9; Schaufeli et al., 2006a) to assess work engagement during the afternoon. Because we focused on work engagement in the afternoon, we removed three items (c.f. Syrek, Kühnel, et al., 2018) that are difficult to answer for this period (e.g., "When I get up in the morning, I feel like going to work"). The final scale contained six items, and every item started with "since the last questionnaire" (i.e., the noon questionnaire). A sample item was, "Since the last questionnaire, I was immersed in my work." Cronbach's alpha was .90.

\section{Control variables.}

In addition to RRSE, pleasant anticipation, and work engagement, we assessed different control variables. First, to demonstrate that pleasant anticipation refers to the future and is different from present positive affect, we controlled for state positive affect. Furthermore, because poor sleep hygiene is related to lower work engagement (Barber et al., 2013), we controlled for sleep quality. Moreover, we introduce pleasant anticipation as an indicator of anticipated resource gain, and therefore, we controlled for present resources at the beginning of the working day. 
Finally, demographics (age, gender) may change the selection of the planned leisure acidity (Brajša-Žganec et al., 2011); thus, we controlled for age and gender.

Sleep Quality (Morning Questionnaire). Sleep quality was measured with one item from the Pittsburgh Sleep Quality Index (Buysse et al., 1989). The participants were asked, "How do you evaluate this night's sleep?" and could indicate their subjective sleep quality on a scale ranging from very bad (1) to very good (4). One item measurement of sleep quality has been predominantly used in previous studies (e.g., Hülsheger, 2016; Kühnel et al., 2018).

Resources (Morning Questionnaire). We used a short questionnaire from Hunter and $\mathrm{Wu}$ (2016), which was developed to measure energy, motivation, and concentration resources with one item each. A sample item was, "What is your current level of motivation?". Cronbach's alpha was .89 .

Positive Affect (Noon Questionnaire). We assessed positive state affect with six items combining positive activating and positive deactivating affect from Kessler and Staudinger (2009). Participants indicated how they felt at present (e.g., "elated," "excited”). Cronbach's alpha was .85 .

\section{Study 1a: Results}

Means, standard deviations and correlation of all variables are presented in Table 2. We used linear hierarchical regression models to test our hypotheses. Pleasant anticipation and RRSE were centred at the mean before calculating the interaction term to analyse the moderation. The control variables were entered in the final step to show that they are not responsible for our results (Becker, 2005). In Hypothesis 1, we suggested that pleasant anticipation of a planned leisure activity is positively related to work engagement in the afternoon. Pleasant anticipation of a planned leisure activity was positively related to work engagement, $B=0.208, S E=0.082$, $p=.013$. Hence, Hypothesis 1 was supported. In addition, the direct relationship between RRSE and work engagement was significant, $B=0.280, S E=0.107, p=.010$. In Hypothesis 2 , we argued that RRSE moderates the positive relationship between pleasant anticipation and work engagement such that the relationship becomes stronger for employees with high RRSE. In contrast to our expectations, RRSE did not moderate the relationship between pleasant anticipation and work engagement, $B=-0.078, S E=0.116, p=.506$. Thus, Hypothesis 2 was rejected. 


\section{Table 2}

Means (M), Standard Deviations (SD) and Correlations of the Variables in Study 1 a

\begin{tabular}{|c|c|c|c|c|c|c|c|c|c|}
\hline Variable & $M$ & $S D$ & 1 & 2 & 3 & 4 & 5 & 6 & 7 \\
\hline 1 Age & 37.54 & 13.21 & & & & & & & \\
\hline 2 Gender $^{\mathrm{a}}$ & 0.55 & 0.50 & -.01 & & & & & & \\
\hline 3 Sleep quality & 2.93 & 0.57 & $.25^{*}$ & -.07 & & & & & \\
\hline 4 Resources & 3.40 & 0.83 & .21 & -.01 & $.30 * *$ & & & & \\
\hline 5 Positive affect & 2.94 & 0.76 & -.06 & .11 & .13 & $.35 * *$ & & & \\
\hline 6 RRSE & 3.07 & 0.77 & .18 & -.08 & .16 & .17 & .16 & & \\
\hline 7 Pleasant anticipation ${ }^{\mathrm{b}}$ & 3.74 & 1.01 & $-.24 *$ & .06 & .03 & .19 & .18 & .05 & \\
\hline 8 Work engagement & 3.05 & 0.81 & .11 & -.04 & .11 & $.36^{* *}$ & $.47 * *$ & $.28 * *$ & $.27^{*}$ \\
\hline
\end{tabular}

Note. $N=85$. RRSE $=$ recovery-related self-efficacy.

${ }^{\mathrm{a}}$ male $=0$, female $=1$.

bof a leisure activity.

${ }^{*} p<.05 .{ }^{* *} ; p<.01$

After entering the control variables (Table 3), the relationship between pleasant anticipation and work engagement remained significant, $B=0.165, S E=0.081, p=.045$; however, the relationship between RRSE and work engagement was non-significant $(B=0.171$, $S E=0.103, p=.101)$. Removing the non-significant interaction from the model with controls only slightly changed the results. 


\section{Table 3}

Results from Linear Modelling to Predict Work Engagement on one Working Day

\begin{tabular}{|c|c|c|c|c|c|c|c|c|c|}
\hline \multirow{2}{*}{ Variable } & \multicolumn{3}{|c|}{ Model 1} & \multicolumn{3}{|c|}{ Model 2} & \multicolumn{3}{|c|}{ Model 3} \\
\hline & $B$ & $S E$ & $p$ & $B$ & $S E$ & $p$ & $B$ & $S E$ & $p$ \\
\hline Pleasant anticipation ${ }^{\mathrm{a}}$ & 0.208 & 0.082 & .013 & 0.196 & 0.084 & .022 & 0.165 & 0.081 & .045 \\
\hline RRSE & 0.280 & 0.107 & .010 & 0.293 & 0.109 & .009 & 0.171 & 0.103 & .101 \\
\hline Pleasant anticipation ${ }^{\mathrm{a}} *$ RRSE & & & & -0.078 & 0.116 & .506 & -0.008 & 0.109 & .943 \\
\hline Age & & & & & & & 0.115 & 0.086 & .185 \\
\hline Gender $^{\mathrm{b}}$ & & & & & & & -0.138 & 0.152 & .367 \\
\hline Sleep quality & & & & & & & -0.051 & 0.096 & .594 \\
\hline Resources & & & & & & & 0.121 & 0.089 & .177 \\
\hline Positive affect & & & & & & & 0.329 & 0.089 & $<.001$ \\
\hline$\Delta R^{2}\left(p\right.$ value change in $\left.R^{2}\right)$ & \multicolumn{3}{|c|}{$.15(.002)$} & \multicolumn{3}{|c|}{$.01(.506)$} & \multicolumn{3}{|c|}{$.20(.001)$} \\
\hline$R^{2}$ & \multicolumn{3}{|c|}{.15} & \multicolumn{3}{|l|}{.15} & \multicolumn{3}{|c|}{.35} \\
\hline
\end{tabular}

Note. $N=86$. RRSE = recovery-related self-efficacy. Age, sleep quality, resources, and positive affect were zstandardized for a better interpretation.

${ }^{\mathrm{a}}$ male $=0$, female $=1$.

bof a leisure activity. 


\section{Study 1b: Method}

\section{Method}

In Study 1b, we used an online diary design. The participants had to be at least 18 years old, work full-time, and have regular working hours (e.g., no shift-work). After signing in for the study, participants chose a week to participate in the diary study and completed the presurvey. The online diary contained three daily questionnaires (morning, noon, and afternoon) from Monday to Friday. Participants were recruited by posting flyers on social media websites, using personal contacts, and three psychology students helped us with data collection as part of one research and two master's thesis projects. Participation was voluntary, and we offered participants $15 €$ for filling in at least seven questionnaires and $20 €$ for completing twelve or more questionnaires out of 15 questionnaires. In sum, 83 of 94 registered participants completed the presurvey $(88.23 \%$ ), and out of these 83 participants, we collected data from 842 measurement points (response rate: $67.63 \%$ ).

\section{Participants}

Participants (65\% females) were, on average, 28.07 years old $(S D=6.75)$. Most of them held a master's degree (33.75\%), finished vocational training (19.28\%), or held a bachelor's degree $(18.07 \%)$. On average, they worked 38.33 hours per week $(S D=6.62)$ and were employed in different occupational sectors, for example, health and social work (20.48\%), information and communication $(15.66 \%)$, or education $(7.23 \%)$. Some participants were in a leadership position (20.48\%), and three employees were self-employed.

\section{Measures}

We applied the same questionnaires as in Study 1a. Because we were interested in RRSE as a stable trait, RRSE was assessed in the presurvey. The other variables were measured from Monday to Friday with the same three questionnaires used in Study 1a: the morning questionnaire (sleep quality, resources), the noon questionnaire (pleasant anticipation, positive affect), and the afternoon questionnaire (work engagement).

\section{Study 1b: Results}

We included participants in the analyses when they completed all three questionnaires on at least two days. Means, standard deviations, reliabilities (Cronbach's alpha), and correlation coefficients are presented in Table 4. Study 1 b provided data on the between-person level (e.g., RRSE) and on the within-person level (e.g., pleasant anticipation), and we used hierarchical linear modelling (HLM; Raudenbush \& Bryk, 2002) to test our hypotheses. The intraclass 
Table 4

Means (M), Standard Deviations (SD), and Correlations of the Variables in Study $1 \mathrm{~b}$

\begin{tabular}{|c|c|c|c|c|c|c|c|c|c|c|}
\hline Variable & $M$ & $S D$ & 1 & 2 & 3 & 4 & 5 & 6 & 7 & 8 \\
\hline 1 Age & 27.21 & 5.36 & & - & - & - & - & - & - & - \\
\hline 2 Gender $^{\mathrm{a}}$ & 0.71 & 0.46 & -.18 & & - & - & - & - & - & - \\
\hline 3 Sleep quality & 2.95 & 0.55 & .08 & .10 & & $.34 * *$ & $.24 * *$ & - & -.04 & $.15^{*}$ \\
\hline 4 Resources & 3.47 & 0.69 & .17 & -.08 & $.44 * *$ & .87 & $.15^{*}$ & - & .07 & $.17 *$ \\
\hline 5 Positive affect & 3.07 & 0.52 & .11 & -.02 & .21 & $.38^{* *}$ & .89 & - & $.15^{*}$ & $.41 * *$ \\
\hline 6 RRSE & 2.93 & 0.92 & .20 & -.06 & .14 & .26 & $.50 * *$ & .88 & - & - \\
\hline 7 Pleasant anticipation ${ }^{\mathrm{b}}$ & 3.87 & 0.60 & .22 & .13 & .11 & $.42 * *$ & $.37 * *$ & .14 & .94 & .08 \\
\hline 8 Work engagement & 3.30 & 0.69 & .12 & .11 & .23 & $.50 * *$ & $.64 * *$ & $.31 *$ & $.43 * *$ & .91 \\
\hline
\end{tabular}

Note. Correlations under the diagonal are between-person correlations $(N=56)$, and correlations above the diagonal are within-person (day-level) correlations $(N=238)$. Italic values in the diagonal show Cronbach's $\alpha$ (mean).

${ }^{\mathrm{a}}$ male $=0$, female $=1$.

${ }^{\mathrm{b}}$ of a leisure activity.

$* p<.05 . * * ; p<.01$

coefficient (ICC) for work engagement was .41. Hence, the ICC demonstrated that work engagement varies within persons, justifying HLM analyses. For HLM, we included only participants who completed at least all three questionnaires on two different days (64 participants). The within-person (day-level) variables (e.g., pleasant anticipation, sleep quality) were group-mean centred, and the between-person (person-level) variables (RRSE, age) were grand-mean centred. Because we tested a cross-level interaction, we estimated a random slope model for pleasant anticipation (Aguinis et al., 2013).

Hypothesis 1 stated that pleasant anticipation of a planned leisure activity is positively related to work engagement. The within-person relationship between pleasant anticipation and work engagement was negative, yet not significant, $\gamma=-0.112, S D=0.070, p=.113$ (Table 5, model 1). Hence, Hypothesis 1 was rejected. However, we found a significant direct relationship between RRSE and work engagement $(\gamma=0.245, S D=0.091, p=.001)$. In Hypothesis 2, we expected RRSE to moderate the relationship between pleasant anticipation and work engagement. The interaction was not significant $(\gamma=0.043, S D=0.066, p=.519$; model 2). Hence, Hypothesis 2 was rejected. In model 3, we added the same control variables as in Study 1a. The results only changed slightly, and, in contrast to Study 1a, age was positively related to work engagement, $\gamma=0.034, S D=0.016, p=.037$. Finally (model 4), we used the grand-mean centred average pleasant anticipation of each participant to include between-person effects (Raudenbush \& Bryk, 2002). The results replicated the results from Study la by showing 


\section{Table 5}

Results from Hierarchical Linear Modelling to Predict Day-Level Work Engagement

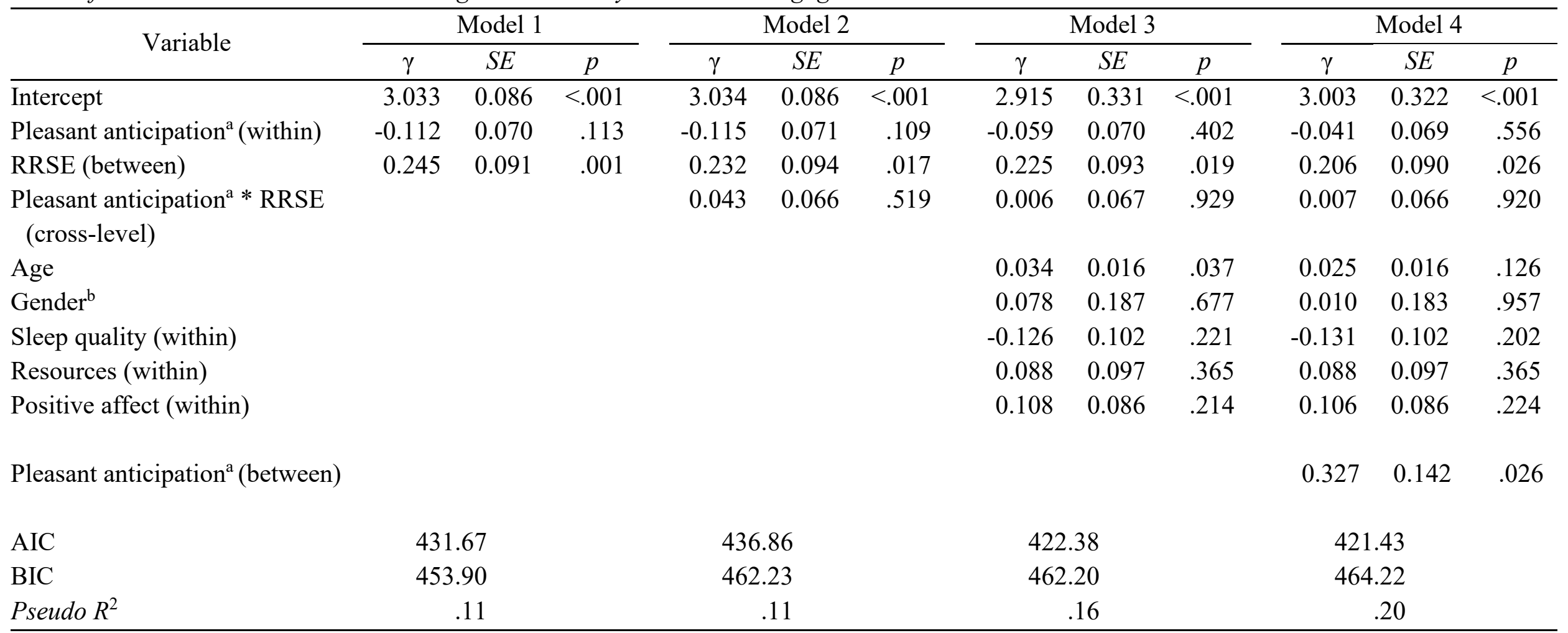

Note. $N=180$ (within-person) and $N=56$ (between-person). $\gamma=$ unstandardized estimates. RRSE = recovery-related self-efficacy. BIC $=$ Bayesian information criterion; AIC = Akaike's information criterion. Pseudo $R^{2}$ was calculated using a formula by Snijders and Bosker (2012).

${ }^{a}$ male $=0$, female $=1$.

${ }^{b}$ of a leisure activity. 
that pleasant anticipation of a planned leisure activity was positively and significantly related to work engagement between persons, $\gamma=0.327, S D=0.142, p=.026$. Our results only slightly changed when adding the between-person effect in model 1 or removing the non-significant interaction from model 3 or model 4.

\section{Discussion}

In two studies, we investigated whether positive anticipation of upcoming leisure activities is related to present work engagement. Based on the COR theory (Hobfoll, 1989; Hobfoll et al., 2018), we used employees' pleasant anticipation of a planned leisure activity as an indicator of an upcoming resource gain, and we assumed that pleasant anticipation is positively associated with work engagement. We further considered RRSE to be a moderator in this relationship and proposed that RRSE strengthens the relationship between pleasant anticipation and work engagement.

We found evidence that pleasant anticipation of a planned leisure activity and work engagement were positively related between persons. Regarding Study 1a, employees with higher pleasant anticipation on one day were more engaged in their work than employees with lower pleasant anticipation. In Study 1b, the between-person relationship indicated that employees with higher average pleasant anticipation across the week reported higher work engagement than employees with lower average pleasant anticipation. Overall, our results are the first hint that pleasant anticipation of a planned leisure activity and work engagement are positively related between persons.

In contrast, pleasant anticipation of a planned leisure activity was not associated with work engagement within persons, i.e., the same employee did not report higher work engagement on days with high pleasant anticipation than on days with low pleasant anticipation. Alternatively, experiencing pleasant anticipation at noon was not related to higher work engagement during the afternoon for the same employee. One explanation may be that pleasant anticipation can include excitement about the planned leisure activity, and, as a result, employees may frequently think of their planned leisure activity, which may draw their attention away from the task at hand. This explanation concurs with previous research, which demonstrated that thoughts of leisure time are negatively related to work engagement (Seibel et al., 2020). Furthermore, one may also speculate that the impact of anticipated resource gains on daily work engagement is small and may be masked by other day-level resources, such as daily job control (Kühnel et al., 2012) or daily self-efficacy (Xanthopoulou et al., 2009). Moreover, we assumed that pleasant anticipation indicates the value of an anticipated resource 
gain, but the type of the anticipated resource gain may also matter for employees' work engagement. The anticipation of a physical leisure activity may relate to work engagement through another mechanism (e.g., expecting physical activation) compared to the anticipation of a low-effort leisure activity (e.g., expecting recovery). Finally, it seems as if there is no unique theoretical process underlying the relationship between pleasant anticipation and work engagement, and the underlying process depends on temporal perspectives and levels of analysis.

In summary, although day-level pleasant anticipation of a planned leisure activity was not related to employees' work engagement, employees with higher pleasant anticipation reported higher work engagement compared to other employees. Therefore, pleasant anticipation (between-persons) may reflect a generally positive view of upcoming leisure time, especially as the between-person differences in Study la referred to average pleasant anticipation across one week. Averaged pleasant anticipation shares similarities with another resource, optimism, which refers to a generally positive future and the expectation of a good outcome (Peterson, 2000; Scheier \& Carver, 1985) and was found to be positively related to work engagement (Xanthopoulou et al., 2007, 2009). In terms of COR theory (Hobfoll, 1989; Hobfoll et al., 2018), our findings may demonstrate that pleasant anticipation of a planned leisure activity could be a resource. However, while employees with high optimism expect a positive future in general, employees with high general pleasant anticipation expect positive leisure activities not only on a specific day, but also in general. Thus, general pleasant anticipation may help employees be more resilient in the workplace.

We further investigated the role of RRSE as a moderator in the relationship between pleasant anticipation and work engagement. In contrast to our assumptions, RRSE did not moderate this relationship. One reason could be that pleasant anticipation is a specific affective reaction referring to one selected planned leisure activity, while RRSE contained employees' overall experiences with past leisure activities. Instead of moderating the relationship between pleasant anticipation and work engagement, RRSE was positively associated with work engagement in both studies. Thus, employees' beliefs about their ability to recover during leisure time could be regarded as a unique resource that did not depend on or interact with specific planned leisure activities. The non-significant relationship between RRSE and pleasant anticipation in Study 1a and the moderate relationship in Study 1b further confirmed that idea. In sum, both RRSE and general pleasant anticipation of a planned leisure activity could be considered as leisure-related resources, which are positively related to work engagement between persons. 


\section{Theoretical Implications}

We investigated the concept of pleasant anticipation and overviewed related constructs. In two studies, we revealed that pleasant anticipation is a unique concept. First, controlling for employees' sleep quality and resources in the morning did not change the positive betweenperson association between pleasant anticipation and work engagement in both studies. Thus, pleasant anticipation could be differentiated from variables indicating present resources, fostering the idea that pleasant anticipation refers to upcoming events. Second, we controlled for employees' state positive affect, and, as the results did not change, demonstrated that pleasant anticipation is more than just a positive affective reaction. Thus, the measurement of pleasant anticipation is distinct from the measurement of positive affect. Finally, we expanded previous research on the pleasant anticipation of future events (e.g., vacation; Nawijn et al., 2013) and anticipation in the working context (e.g., the anticipation of workload; DiStaso \& Shoss, 2020) and showed that research on the anticipation of leisure activities is also important. In sum, pleasant anticipation is a promising construct for examining the value of future events, as it combines an affective reaction with information about the future event.

The different findings for within- and between-person relationships show that this differentiation is necessary (McCormick et al., 2018). Furthermore, it is important to note that interpreting the between-person relationship depends on the time frame of a study. For instance, in Study 1a, the between-person relationship is based on differences in work engagement between persons on one working day; yet, in Study 1b, the between-person relationship refers to the difference across a working week. In addition, when research focuses on hourly changes on one working day, the between-person relationship indicates averaged differences across one day (Seibel et al., 2020; Syrek, Kühnel, et al., 2018). Thus, the time frame of a study should be carefully chosen to get a meaningful interpretation of between-person relationships.

Overall, both studies supported the idea that resources are crucial to employees' work engagement (Lesener et al., 2019). Interestingly, the two leisure-related resources were not or only moderately correlated, indicating that they refer to different types of resources. While RRSE shares similarities with general self-efficacy and emerges from past experiences (Bandura, 1977, 1994), general pleasant anticipation may arise from a (positive) view of future leisure time and may relate to optimism. Consequently, as employees could rely on past experiences and future leisure time when managing their resources at work, both types of leisure-related resources jointly predict work engagement. 


\section{Practical Implications}

Our study showed that pleasant anticipation and work engagement were positively related between persons. Thus, it may be beneficial for employees to have an optimistic view of their leisure time in general. This view could be fostered in two ways: First, planning leisure activities with opportunities to (re)gain resources may help employees experience pleasant anticipation every day, which amounts to an overall positive view in the long run. Second, as pleasant anticipation shares similarities with the ability to savour future events before they occur (Bryant \& Veroff, 2006b), we assume that employees could learn to experience pleasant anticipation. Savouring interventions ask participants to think actively about a positive future event and enhance their well-being (for a meta-analysis, see J. L. Smith et al., 2014). These interventions could be adapted to foster an overall positive view of leisure time. For instance, Quoidbach et al. (2009) demonstrated that positive future mental time travel enhanced participants' happiness. Similarly, the experience of pleasant anticipation of a planned leisure activity may help increase employees' work engagement.

\section{Future Research and Limitations}

Our studies are not without limitations. First, we asked employees to think about positive and negative planned leisure activities, yet few employees mentioned less positive leisure activities, such as tidying up or doctor's appointments. Thus, average pleasant anticipation was high. We had expected that more employees would indicate lower pleasant anticipation because of less positive planned leisure activities. However, as we concentrated on anticipated resource gains indicated by high pleasant anticipation, less positive planned leisure activities were not the focus of our research question. Nevertheless, future research should consider anticipated resource losses and use a quasi-experimental design, asking employees to think of either positive or negative planned leisure activities.

Second, to draw conclusions about employees' pleasant anticipation of a planned leisure activity, we had to ask them to think about their leisure time. This request could be regarded as a short intervention that might have affected employees' work engagement across the week. However, typical short interventions last longer (e.g., 5-7 min; Lischetzke et al., 2015), and our method is very common to investigate future events and thoughts (e.g., Barsics et al., 2016; Seibel et al., 2020). Thus, we assume that our results did not depend on our measurement method. To discard the explanation that thinking about a positive (future) event regulates between-person differences in work engagement, future research should compare the evaluation of positive past and future leisure activities. 
Finally, we suggested that pleasant anticipation, as an indicator of an anticipated resource gain, is directly related to employees' work engagement. Past research also focused on direct relationships between resources and work engagement (Kühnel et al., 2012; Xanthopoulou et al., 2009); however, future research could investigate the mechanisms that may connect pleasant anticipation and work engagement. At the within-person level, investigating mediators may help to explain the non-significant results. For instance, while pleasant anticipation could enhance employees' work engagement through an expectation of recovery, pleasant anticipation may diminish work engagement through an enhanced frequency of thoughts of the planned leisure activity.

\section{Conclusion}

Our research revealed that pleasant anticipation of a planned leisure activity relates positively to work engagement between persons but not within persons. Furthermore, we found evidence that recovery-related self-efficacy (RRSE) was positively related to work engagement. In sum, a positive view of upcoming leisure time in general (i.e., high general pleasant anticipation of leisure activities) and positive experiences with past leisure time (high RRSE) may be leisurerelated resources that benefit employees' work engagement.

\section{Acknowledgement}

We thank Julia Ramming and the students of the research course for their help with the data collection for Study 1a. We further thank Anastasiya Mykolenko, Franziska Ruppert, and Katharina Schweiger for recruiting the participants for Study $1 b$.

\section{Funding}

Study $1 \mathrm{~b}$ was supported by a grant from the Faculty of Human Sciences at the University of Wuerzburg.

\section{Disclosure statement}

We have no conflicts of interest to disclose. 


\title{
STUDY 2: GET A TASTE OF YOUR LEISURE TIME - THE RELATIONSHIP BETWEEN LEISURE THOUGHTS, PLEASANT ANTICIPATION, AND WORK ENGAGEMENT
}

\author{
Sebastian Seibel ${ }^{\mathrm{a}}$, Judith Volmer ${ }^{\mathrm{a}}$ and Christine J. Syrek ${ }^{\mathrm{b}}$ \\ ${ }^{a}$ Work and Organizational Psychology Group, Otto-Friedrich University of Bamberg, \\ Bamberg, Germany; \\ ${ }^{b}$ Business Psychology, University of Applied Science Bonn-Rhein-Sieg, Rheinbach, Germany
}

This is an Accepted Manuscript of an article published by Taylor \& Francis in European Journal of Work and Organizational Psychology on 16.09.2020, available at:

http://www.tandfonline.com/10.1080/1359432X.2020.1804875

\begin{abstract}
During the working day, employees do not only think of their work but also occasionally of their upcoming leisure time. Accordingly, we introduce two constructs, namely thoughts of leisure time (ToLT) and thoughts of a planned leisure activity (ToPLA). We assumed that employees report more ToLT/ToPLA at the beginning and the end of the working day. We further hypothesized that employees with higher pleasant anticipation of a planned leisure activity generate more ToPLA. As leisure thoughts distract attention from work, we expected a negative relationship between ToLT/ToPLA and work engagement within one hour and across the working day. Regarding the subsequent hour, we assumed that when the leisure plan is positive/negative, the relationship between ToPLA and work engagement is positive/negative. We conducted an hourly online-survey across one working day $(N=89$ employees, 438 measurement points). Our results revealed the expected time trend for ToLT/ToPLA and a positive relationship between pleasant anticipation and ToPLA. We further found negative relationships between ToPLA and work engagement (within one hour) and between ToLT and work engagement (across the day). Contrary to our expectations, for positive leisure plans, the relationship between ToPLA and work engagement in the subsequent hour was negative.
\end{abstract}

Keywords: leisure thoughts; pleasant anticipation; leisure time; work engagement; hourly measurement 


\section{Theoretical Background}

During their leisure time, many employees cannot detach from their work and still report workrelated thoughts (e.g., Flaxman et al., 2018; Querstret et al., 2017; Weigelt et al., 2019b). For instance, employees think of unfinished tasks (Weigelt et al., 2019b) and negative interactions with customers (Wang et al., 2013). Furthermore, negative social events at work spill over into leisure time and increase employees' negative affect as employees ruminate about these work events (Volmer et al., 2012; Volmer, 2015). Thus, employees think of their work during their leisure time. Flipping the coin, we suggest that employees also think of their leisure time (e.g., of some planned leisure activity) during their working day. We refer to such thoughts as leisure thoughts.

Leisure thoughts can be described as off-task thoughts (Beal et al., 2005; Gardner et al., 1989; Kanfer \& Ackerman, 1989), as employees are usually encouraged to think of their work (i.e., on-task thoughts) and not of their leisure time (i.e., off-task thoughts). Off-task thoughts are important for maintaining behaviour (e.g., goal selection, making plans) by keeping higherorder goals and upcoming future events in mind (Atance \& O'Neill, 2001; Marchetti et al., 2016; Martin \& Tesser, 1996; Mason \& Reinholtz, 2015; Szpunar, 2010). Although research has shown that one content of off-task thoughts is leisure time (Barsics et al., 2016; D'Argembeau et al., 2011), leisure thoughts themselves have received little attention. Past research on the consequences of off-task thoughts did not differentiate between leisure thoughts and other types of off-task thoughts (e.g., Dimitrova et al., 2015; Kanfer \& Ackerman, 1989) and revealed that off-task thoughts impair task performance (for a meta-analysis, see Randall et al., 2014). Contrary to this stream of research, Dane (2018) proposed positive consequences of off-task thoughts on employees' task performance if they remind employees of future goals. Yet the question remains as to whether leisure thoughts have positive or negative consequences for employees' work engagement. We focus on work engagement as work engagement is both, a subjective motivational state (Bakker et al., 2014) and an antecedent to objective performance (Hakanen et al., 2008; Salanova et al., 2010). More specifically, work engagement is a positive motivational reaction (Xanthopoulou et al., 2009) which includes the feeling of vigour, dedication, and absorption concerning one's work (Schaufeli \& Bakker, 2004).

In the present study, we address three topics and contribute to the literature in several ways. First, we introduce the construct leisure thoughts and examine their occurrence over the working day. We refer to construal level theory (Trope \& Liberman, 2010; Wiesenfeld et al., 2017) and assume that employees generate more leisure thoughts at the beginning and at the end of the working day because work and leisure time are more proximate. As we investigate 
the time trends of different types of thoughts (leisure thoughts, on-task thoughts, thoughts of other things), we extend the research on thoughts at work by adding a temporal perspective, enabling us to understand whether employees think of their work from the beginning to the end of their working day to the same degree at every hour or whether there is a smooth transition from work to leisure time and vice versa. Second, we posit that employees differ in their feelings of excitement and joy due to an upcoming planned leisure activity. We refer to this unique experience as pleasant anticipation and hypothesize that employees with higher pleasant anticipation think more frequently of a planned leisure activity. Since research on pleasant anticipation has so far been limited to special events like Christmas (Syrek, Weigelt, et al., 2018) or holidays (Nawijn et al., 2013), we shed light on pleasant anticipation of more usual events like daily leisure time. Third, we posit that leisure thoughts have both positive and negative consequences for work engagement. On the one hand, as leisure thoughts might distract employees from their current tasks, we assume that the frequency of leisure thoughts is negatively related to work engagement within one hour and across the working day. On the other hand, we build on conservation of resources (COR) theory (Hobfoll, 1989; Hobfoll et al., 2018) and argue that thoughts of a planned leisure activity either indicate an upcoming resource gain or an upcoming resource loss. We assume that when leisure plan valence is positive, thoughts of a planned leisure activity are associated with higher work engagement. In contrast, we suppose that these thoughts are negatively related to work engagement, when leisure plan valence is negative. As leisure thoughts may initially distract employees from the task at hand, we assume that these relationships emerge for work engagement in the subsequent hour. With our research, we aim to ascertain whether an anticipated resource gain/loss in the future relates to present work engagement. This contributes to the research on leisure plans (Dumas \& PerrySmith, 2018) and, in a broader sense, to the research on recovery (Sonnentag et al., 2017; e.g., Sonnentag, 2018), as we assume that the mere anticipation of leisure activities may be important for work engagement. Our research model is presented in Figure 1.

\section{Definition of Leisure Time and Leisure Thoughts}

To understand the concept of leisure thoughts, we first propose a short definition of leisure time. In general, leisure time refers to time free from work with no obligations, which employees can spend as they choose (S. R. Parker \& Smith, 1976; Thierry \& Janson, 1998). However, it is difficult to define leisure time (Geurts \& Demerouti, 2003; Kabanoff, 1980), as "the judgement where work leaves off and leisure begins is usually a subjective one" (S. R. Parker \& Smith, 1976, p. 41). For instance, some employees regard cooking in their non-work 
Figure 1

Research Model

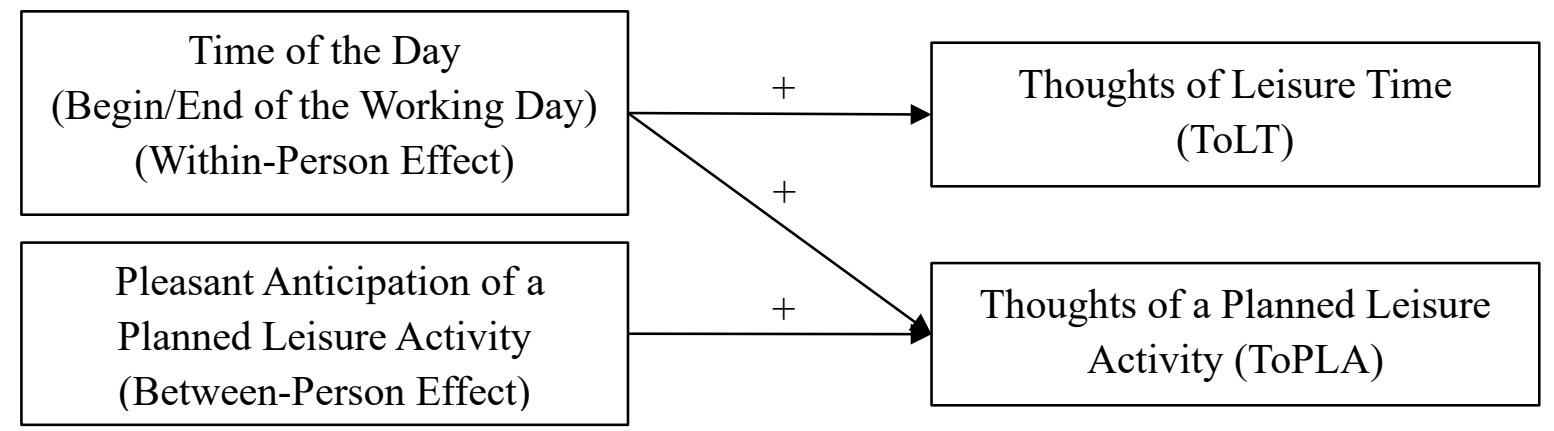

Thoughts of Leisure Time (ToLT)

Thoughts of a Planned Leisure Activity (ToPLA)

(Between-Person Effect)

Work Engagement

(day-level)

Thoughts of Leisure Time (ToLT)

Thoughts of a Planned Leisure Activity (ToPLA)

(Within-Person Effect; concurrently)

Thoughts of a Planned Leisure Activity (ToPLA)

(Within-Person Effect; lagged)

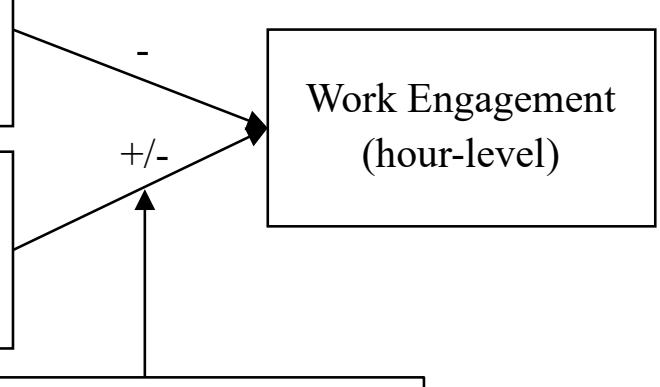

Leisure Plan Valence

(Between-Person; cross-level)

Note. Upper part: antecedence of leisure thoughts. Lower part: consequences of leisure thoughts.

time as an obligatory household activity, while other employees enjoy cooking. Thus, employees have their subjective definitions of leisure time. We therefore use a broad definition of leisure thoughts and leisure time. More precisely, we define leisure time as a non-working time before and after employees' regular working hours. During this leisure time, employees engage in various activities, all of which are potentially leisure activities, and each employee has his or her opinion regarding which activities are leisure activities (Newman et al., 2014). Starting with this broad definition, we developed the construct of leisure thoughts. 
We define leisure thoughts as a special type of future-oriented off-task thoughts ${ }^{1}$ occurring at work and not related to the task at hand. We further distinguish between two different types of leisure thoughts. We refer to the first type as Thoughts of Leisure Time (ToLT) because they include all kinds of thoughts employees have of their leisure time. More specifically, we define ToLT as future-oriented thoughts of today's leisure time. Some examples for these thoughts are "What can I do to recover in the evening?", "I do not know what to cook for dinner", and "I will be very tired after work". These examples show that ToLT are not specific about an activity and may be very general in their content. The content of ToLT may include many different themes and/or leisure activities, which vary across the working day.

We term the second type of leisure thoughts Thoughts of a Planned Leisure Activity (ToPLA) as they refer specifically to a planned leisure activity. ToPLA are future-oriented thoughts of a specific plan that employees have in mind for their leisure time. These plans include specific leisure activities, for instance, meeting with friends, physical activities, or a doctor's appointment. Corresponding ToPLA for these examples may be "Today, I will meet up with friends", "I will go for a walk in my leisure time", and "I have a doctor's appointment after work". However, the content of ToPLA over the working day could be diverse and they have in common that they refer to the same planned leisure activity. In contrast to ToLT, the content of ToPLA refers to only one leisure activity as long as the plan for leisure activity does not change. Furthermore, ToPLA are a subtype of ToLT, and when employees have more than one leisure plan, ToPLA for the first plan, ToPLA for the second plan, and so on may exist.

\section{Frequency of Leisure Thoughts During the Working Day}

The first aim of this study is to investigate the frequency of leisure thoughts. Support for the existence of ToLT and ToPLA comes from research on future-oriented thoughts (for a review, see Aspinwall, 2005). In general, most (35\%) future-oriented thoughts are about leisure time (D'Argembeau, 2018) and 25\% occur at work (Barsics et al., 2016). In more detail, individuals report on average 59 future-oriented thoughts per day, resulting in about one thought every 16 minutes (D'Argembeau et al., 2011). These off-task thoughts may be triggered for different reasons (e.g., unattained goals, negative mood; Mrazek et al., 2011; Smallwood \& Schooler, 2015; Watkins, 2008), yet research did not address the question when more or less off-task thoughts occur during an employee's working day (Beal et al., 2005; Merlo et al., 2018). Thus, it remains unclear whether off-task thoughts might follow a systematic time trend.

\footnotetext{
${ }^{1}$ We use the term off-task thoughts. However, leisure thoughts can also be described as one special type of mind wandering, spontaneous thoughts or daydreaming (for an overview, see Marchetti et al. , 2016).
} 
Building on construal level theory (Trope \& Liberman, 2010; Wiesenfeld et al., 2017), we assume that both ToLT and ToPLA will occur more often at the beginning and at the end of the working day. Construal level theory implies that psychological distance (temporal, spatial, social, hypothetical) changes human thoughts and perceptions (Trope \& Liberman, 2010). Psychological distance describes the subjective feeling that something (e.g. objects, events) is closer or farther away from the self (Trope \& Liberman, 2010). According to Liberman and Trope (1998), temporal distance indicates that when a future event gets closer in time, mental representations change from more abstract levels of construal (prototypical, general, overarching goals) to more concrete levels of construal (specific, detailed, focus on observable behaviour).

At the beginning of the working day, when the temporal distance from employees' past leisure time (e.g. having breakfast) is very short, construal level theory predicts that employees have detailed representations of their leisure time (Trope \& Liberman, 2010). Detailed representations with a great deal of contextual information are more likely to be retrieved from memory (Chu et al., 2003; Howard \& Kahana, 1999). For instance, employees may have talked with their spouse about dinner or who will do the shopping just before leaving for work. Thus, there are more cues at the beginning of the working day to trigger employees' thoughts of their upcoming leisure time (ToLT/ToPLA).

At the end of the working day, when the temporal distance to employees' upcoming leisure time (e.g. doing the shopping) is shorter, employees have more detailed representations of their upcoming leisure time as indicated by construal level theory (Trope \& Liberman, 2010; Wiesenfeld et al., 2017). Again, ToLT/ToPLA are more likely to be retrieved from memory due to the more detailed mental representation (Chu et al., 2003; Howard \& Kahana, 1999). This concurs with research on spontaneous thoughts, which serve as a reminder of upcoming events (Marchetti et al., 2016). Furthermore, thoughts of an upcoming event occur more frequently when this event draws closer in time (D'Argembeau et al., 2011). In sum, we assume a U-shaped time trend for the frequency of ToLT/ToPLA during the working day.

Hypothesis 1: There is a U-shaped time trend for the frequency of ToLT/ToPLA during the working day (within-person).

\section{Positive Relationship between Pleasant Anticipation and the Frequency of ToPLA}

As explained above, we argue that time of the day may explain why employees generate more ToLT/ToPLA at the beginning and at the end of the working day. Also, we suggest that the content of the leisure thoughts matters. Thus, we take a closer look at ToPLA, as they refer to 
a planned leisure activity, which can be described in more detail. We suppose that high pleasant anticipation of the planned leisure activity is positively related to the frequency of ToPLA.

We define pleasant anticipation as a positive affective reaction (e.g. joy, excitement) which individuals experience when looking forward to a positive future event. This is in line with Baumgartner, Pieters, and Rik (2008) who stated: “[...] joy about a future event is called 'Vorfreude' in German and 'voorpret' in Dutch (literally pre-joy), and it may be translated as pleasant anticipation" (p. 695). Although the affective reactions themselves (e.g., joy, excitement) may be classified within existing models of affect (e.g., circumplex model; Russell, 1980; Seo et al., 2004), pleasant anticipation is more than a mere affective reaction. First, pleasant anticipation always refers to a positive future event and cannot be experienced without a positive "target" event (whereas individuals may feel happy without knowing why). Second, pleasant anticipation is only an experience about positive future events (unlike happiness which may also refer to past events). Third, the occurrence of the positive future event must be certain (compared to hope, which indicates uncertainty concerning a future event; Roseman, 1996). Finally, pleasant anticipation combines affective reactions such as excitement (because individuals cannot wait for the future event) and happiness (because the future event is positive). Excitement and happiness are only examples of possible affective reactions, as different affective reactions are used to describe the anticipation of positive future events. For instance, Kong, Tuncel, and Parks (2011b) refer to happiness, Baumgartner and colleagues (2008) refer to joy, and sometimes no distinct affective reaction is mentioned (e.g., Graham et al., 2019; Sonnentag, Mojza, et al., 2008).

As noted above, one antecedent for pleasant anticipation is that the future event (e.g. the planned leisure activity) has a positive valence. This valence rating is a general evaluation of whether the future event is expected to be positive or negative and not an affective reaction. As information about the affective reaction in the present is missing, a positive valence rating is not sufficient to experience pleasant anticipation; however, it is a necessary precondition. For instance, we assume the valence of the payment of one's monthly salary may be very positive, yet only a few employees experience pleasant anticipation. We think that they will not normally feel excited because of their payday or think often about it, although the valence is very positive. In contrast, we suggest that when pleasant anticipation of a future event is high, employees think more often about this event (e.g., their planned leisure activity).

According to construal level theory, not only the temporal distance but also the hypotheticality changes our mental representations (Trope \& Liberman, 2010; Wiesenfeld et al., 2017). Hypotheticality refers to the likelihood of occurrence and the desirability of an event. 
If hypotheticality decreases, mental representations will change from a general level of construal to a concrete level of construal (Wiesenfeld et al., 2017). Therefore, we posit that when employees experience high pleasant anticipation of their planned leisure activity, the content of their ToPLA will be more concrete. As a result, the frequency of ToPLA will increase, as the planned leisure activity is more likely to be retrieved from memory (Chu et al., 2003; Howard \& Kahana, 1999).

This is in line with research by Klinger (2013), who showed that higher valued goals evoke more off-task thoughts about these goals. Furthermore, the frequency of future-oriented thoughts increases if their content is relevant to the individual (Newby-Clark \& Ross, 2003; Szpunar, 2010). We assume that high pleasant anticipation indicates that the leisure activity is desirable and relevant to employees. For example, pleasant anticipation may be higher for meeting up with friends than for watching TV at home. Hence, there will be more ToPLA for meeting friends than watching TV. In sum, we suggest that employees experiencing more pleasant anticipation of a planned leisure activity report more ToPLA.

Hypothesis 2: There is a positive relationship between pleasant anticipation of a planned leisure activity and the frequency of ToPLA across the working day (between-person).

\section{Negative Relationship between Leisure Thoughts and Work Engagement}

In line with existing research on the negative consequences of off-task thoughts for task performance (e.g., Beal et al., 2005; Kanfer \& Ackerman, 1989; Randall et al., 2014), we assume that ToLT/ToPLA also impair work-related outcomes (e.g. training performance, work engagement). For instance, Wallace, Edwards, Shull, and Finch (2009) provided evidence that low task focus (i.e., more off-task thoughts) was related to poor performance both in a laboratory and in a field study.

One explanation for the negative consequences of ToLT/ToPLA on performance and work engagement may be that leisure thoughts are interruptions hindering employees from performing well (Baethge \& Rigotti, 2013). When employees think of their leisure time, their attention is distracted from the task at hand and the focus of attention changes (Beal et al., 2005; Merlo et al., 2018). As highly engaged employees must be fully concentrated (Bakker et al., 2008), we expect that work engagement is very sensitive to changes in employees' focus of attention. We therefore posit that employees report lower work engagement within hours of high frequency of ToLT/ToPLA.

Furthermore, the frequency of ToLT/ToPLA may not only differ within employees between different hours but also between different employees across the working day. For 
instance, an employee might only generate a few and brief ToLT/ToPLA every hour. However, this employee was often distracted from his/her work across the working day, which may result in low overall work engagement. Thus, we expect that employees with a great deal of ToLT/ToPLA across the working day will report lower work engagement than employees with less ToLT/ToPLA.

Hypothesis 3a: There is a negative relationship between the frequency of ToLT/ToPLA and work engagement within one hour (concurrently within-person).

Hypothesis 3b: There is a negative relationship between the frequency of ToLT/ToPLA and work engagement across the working day (between-person).

\section{The Moderating Role of Leisure Plan Valence}

Contrary to most research on off-task thoughts (Beal et al., 2005; Kanfer \& Ackerman, 1989; Randall et al., 2014; Wallace et al., 2009), we further posit that ToPLA may have positive consequences for employees' work engagement. We draw on COR theory (Hobfoll, 1989; Hobfoll et al., 2018) and suggest that when the planned leisure activity is positive, ToPLA indicate a higher upcoming resource gain and therefore are positively related to employees' work engagement. In contrast, we also assume that when the planned leisure activity is negative, ToPLA indicate a higher upcoming resource loss and will be negatively related to employees' work engagement.

We define resources as anything having a positive value for the individual, linked to resilience, and necessary for controlling the environment and attaining one's goals (Halbesleben et al., 2014; Hobfoll et al., 2003). Leisure time is a well-known opportunity for employees to gain resources (e.g., Oerlemans et al., 2014; Rook \& Zijlstra, 2006; Sonnentag, 2001), as engaging in positive leisure activities offers them the opportunity to recover (i.e., foster/maintain resilience), experience control over their environment, and/or to achieve private goals. We therefore assume that a positive leisure plan valence represents an anticipated resource gain. Conversely, other leisure activities (e.g. cleaning, administrative tasks) that offer no opportunities to recover, may impede the experience of control and/or may hinder employees from reaching their goals. Thus, we suggest that a negative leisure plan valence indicates an upcoming resource loss.

Anticipated resource gains/losses may relate to employees' work engagement, as not only the actual situation but also the anticipation of an upcoming event is important for resource management (Aspinwall \& Taylor, 1997). In general, employees need resources to maintain their work engagement (Bakker et al., 2014; Bakker \& Demerouti, 2017) and COR theory 
explains employees' resource management at work (Hobfoll et al., 2018). Over the course of a working day, employees' resources decrease, as they have to deal with a variety of mental and/or physical tasks (Meijman \& Mulder, 1998; Trougakos \& Hideg, 2009). Before their resources fall below a certain resource limit, employees enter a defensive mode, stop investing resources and try to conserve their remaining resources (Hobfoll et al., 2018). We expect that when employees anticipate a higher resource gain/loss, they may enter this state later/earlier, as they consider the upcoming resource gain/loss in their current resource management. We assume that when employees anticipate a higher resource gain, they may become more engaged, as they can invest more resources until they reach their resource limit. Conversely, when employees anticipate a higher resource loss, they may have a lower resource limit and can invest fewer resources to become engaged. This concurs to COR theory, which states that the threat of a resource loss (i.e. an anticipated resource loss) has consequences for individuals similar to those of an actual resource loss (Gorgievski \& Hobfoll, 2008a; Hobfoll, 2001a). For instance, Niessen and Jimmieson (2016) showed that an anticipated resource loss impairs work performance. In the same vein, we posit that an anticipated resource gain/loss also relates to employees' work engagement under certain conditions.

We hypothesize that ToPLA play an important role in this resource management process, as ToPLA remind employees of either an anticipated resource gain or an anticipated resource loss. For instance, when employees think more frequently of a positive/negative planned leisure activity, this anticipated resource gain/loss will be experienced as more concrete. Consequently, we assume that the anticipated resource gain/loss has a higher impact on the resource management process. To take both positive and negative leisure plans into account, we suggest that leisure plan valence moderates the relationship between ToPLA and work engagement such that the relationship is positive for a positive leisure plan valence (higher anticipated resource gain) and negative for a negative valence (higher anticipated resource loss). However, we expect that the effects on work engagement do not manifest immediately, as resource gains take time to occur (Hobfoll et al., 2018) and employees may smoothly reduce their work engagement when they reach their resource limit. Moreover, as we have argued that leisure thoughts are a type of off-task thoughts, ToPLA should be negatively related to work engagement within the same hour independently of the leisure plan valence. Therefore, we focus on the relationship between ToPLA and work engagement in the subsequent hour.

Hypothesis 4: Leisure plan valence moderates the relationship between ToPLA within one hour and work engagement in the subsequent hour, such that the relationship is 
positive when leisure plan valence is positive and negative when leisure plan valence is negative.

\section{Method}

We conducted an online-survey among employees in Germany. Participants were recruited with printed flyers distributed in a local town (e.g., at doctors' offices) and digital flyers posted on several social media platforms and sent to personal contacts. Two students also contributed to the data collection as part of their thesis work (for the use of student-recruited samples, see Wheeler et al., 2014). Participation was voluntary, and we offered employees detailed feedback on their work engagement during the working day combined with information about how to stay engaged. Participants had to be over 18 and work about eight hours between 6:00 a.m. to 6:00 p.m. (i.e., work full-time on the day of the participation, no shift workers).

We chose a design with hourly measurement points across one working day (cf. Syrek, Kühnel, et al., 2018). Either Tuesday or Wednesday was selected as the day of the survey, as research showed that for other days employees report special characteristics such as lower mood on Mondays (e.g., Areni, 2008; Larsen \& Kasimatis, 1990) or less workplace incivility on Fridays (Nicholson \& Griffin, 2017). We moreover assumed that more specific leisure activities exist for the middle of the week and that planned leisure activities on Fridays differ from plans on other days as they may include the weekend.

All questionnaires described below were part of one online-survey consisting of a presurvey, thirteen hourly questionnaires, and one questionnaire in the next morning. In the presurvey, we assessed the demographic variables, and participants were instructed to choose a typical working day for their participation (Tuesday or Wednesday within the next seven weeks). On the chosen day, participants received e-mails with links to short questionnaires every hour from 6:00 a.m. to 6:00 p.m. Each questionnaire was available for ten minutes to keep the time between the hourly questionnaires constant. A total number of 106 participants completed 606 hourly questionnaires $(M=5.78, S D=3.24)$. Regarding the average weekly working time of 38.65 hours $(S D=8.51)$, we expected a maximum number of nine questionnaires per day. This resulted in a total response rate of $67.34 \%$. Although this response rate is in line with those of other studies (e.g., Demerouti \& Peeters, 2018), the low response rate could be the result of the restrictive time window for answering the questionnaire. In the next morning, participants received the last questionnaire, which we used to check whether they had engaged in their planned leisure activity. 


\section{Participants}

Demographic information was available for all but five participants, who did not complete the pre-survey. Participants' average age was 35.11 ( $S D=13.70$, ranging from 18 to 64$)$ and $60 \%$ were female. Regarding education, 27 participants had a master's degree, 18 had a bachelor's degree, 23 had completed vocational education, 12 participants had completed higher vocational education and 19 participants were still involved in vocational training. Two participants reported that they had not completed an apprenticeship or other education. Most participants were in a relationship (71.7\%) and $16.1 \%$ had at least one child. Employees worked in various occupations and business sectors, e.g. public administration (14\%), social and health care services $(13 \%)$, business services $(13 \%)$, or other services $(13 \%)$, and in the manufacturing sector (12\%). Ten participants were self-employed and 20.8\% were in managerial positions.

\section{Measures}

We assessed leisure thoughts and work engagement in every hourly questionnaire except for two questionnaires. In the first questionnaire at the beginning of the working day (available until 10:00 a.m.), participants were only asked to describe a specific, planned leisure activity and to report the intensity of pleasant anticipation of that leisure activity as well as leisure plan valence. Furthermore, we did not assess work engagement when participants indicated that they had been on a lunch break.

\section{Pleasant Anticipation and Leisure Plan Valence}

The first questionnaire at the beginning of the working day focused on the planned leisure activity and followed three steps. First, in a few words, participants described a specific, planned leisure activity for their upcoming leisure time. Examples were "watching TV", "meeting up with friend", or "playing volleyball". Second, participants rated their pleasant anticipation of the planned leisure activity on a 5-point Likert scale $(1=$ totally disagree; $5=$ totally agree). To assess pleasant anticipation, we referred to our definition and developed a new scale with four items. These items were: "I cannot wait to put my leisure activity into action", "I am looking forward to my leisure activity", "I feel pleasant anticipation about my leisure activity", and "I have a positive feeling while thinking about my leisure activity". Cronbach's alpha was .92. Third, we used the self-assessment manikin (Bradley \& Lang, 1994) to measure leisure plan valence. The instruction read "Please choose one of the following pictures which best describes your leisure activity". As the self-assessment manikin (Bradley \& Lang, 1994) was initially devolved as an alternative measurement for the semantical 
potential, the scale is bipolar. Participants were asked to select one of seven manikins which ranged from very negative (1) through neutral (4) up to very positive (7).

\section{Leisure Thoughts}

To develop a measure for ToLT/ToPLA, we focused on measurements for on- and off-task thoughts (Gardner et al., 1987; Kanfer et al., 1994; Wallace \& Chen, 2005). Due to our hourly design, we needed a very short measure. Gardner and colleagues (1987) developed a singleitem measurement of both on- and off-task thoughts which did not show important differences in construct validity compared to a multiple-item measurement (Gardner et al., 1998). We created four items based on their measurement with additional information in parentheses to make sure that all participants understood our conceptualisation of ToLT/ToPLA. Participants were asked how frequently they thought of "today's leisure time (thoughts related to events/activities in your upcoming leisure time)" (ToLT), and "today's leisure plan [here the planned leisure activity from the first questionnaire was inserted]" (ToPLA). Moreover, participants indicated how often they thought about their "work (thoughts, which were necessary to get the job done)" (on-task thoughts), and "other things (past events, far-future events, etc.)". The question about "other things" was used to differentiate ToLT and ToPLA from off-task thoughts in general. All items referred to the last hour and participants could answer each item on an abstract rating scale ranging from 1 (not at all) to 10 (all the time). To make sure that our measurement fitted into our broad definition of leisure thoughts and leisure activities, we conducted interviews with 30 employees from another sample and asked them to classify different leisure activities. The results confirmed our broad definition of leisure time and leisure activities. For instance, all employees agreed that physical activities are leisure activities (100\%). However, employees differed in their conceptions of doing the laundry (47\%) or running errands $(67 \%)$ as leisure activities.

\section{Hourly work engagement}

We used an adapted version of the Utrecht Work Engagement Scale (Schaufeli et al., 2006b). Hourly work engagement has previously been assessed with two items each for vigor, dedication, and absorption (Syrek, Kühnel, et al., 2018). A sample item was "In the last hour, I felt bursting with energy". As absorption describes a state of being fully concentrated (Bakker et al., 2008) and could be very sensitive to changes in attention (e.g. a change from on-task thoughts to leisure thoughts), we added an additional absorption item from the original scale ("In the last hour, it was difficult to detach myself from my work."). All items were answered 
on a 5-point Likert scale $(1=$ totally disagree; $5=$ totally agree $)$. Cronbach's alpha ranged from .80 to .95 .

\section{Results}

We used R and the package NLME (Pinheiro \& Bates, 2000) to analyse our data. We followed the procedure by Bliese and Ployhart (2002) for modelling a dynamic time trend for work engagement and the four different thoughts (ToLT, ToPLA, on-task thoughts, and thoughts of other things). To check for within-person effects, predictors on an hourly level were personmean centered. As we were also interested in between-person effects, we entered the grandmean centered person-mean (i.e., a person's day-level) into our model. Pseudo $R^{2}$ was calculated using a formula proposed by Snijders and Bosker (Snijders \& Bosker, 2012, p. 112) representing the total variance explained compared to the null model. We did not control for work engagement in the previous hour, as we were interested in the relationship between leisure thoughts and hourly levels of work engagement and not in the relationship between leisure thoughts and the change in work engagement from one hour to another (which the results indicate when controlling for previous hour's work engagement). We moreover decided to analyse the different types of thoughts separately, as a high frequency of one type of thoughts (e.g. ToLT) means that there was less time for other types of thoughts (e.g. on-task thoughts).

\section{Descriptive Analyses}

We followed common recommendations for the analysis of diary studies and removed 17 from a total of 106 participants who responded to fewer than two hourly questionnaires (Mehl, 2012). Our final sample consisted of 89 participants. Means, standard deviations, and correlations are presented in Table 1. On-task thoughts were the most frequent type of thoughts during the working day $(M=7.54, S D=1.46)$, while the average frequency of all three other types of thoughts was fairly low $(M=3.51, S D=1.60)$. Also, on-task thoughts were positively correlated with work engagement, both at the between-person level $(r=.41, p<.001)$ and at the within-person level $(r=.36, p<.001)$. Employees reported mostly positive und joyful leisure activities, which is reflected in the descriptive statistics for pleasant anticipation $(M=3.97, S D=0.85)$ and leisure plan valence $(M=5.98, S D=1.11)$. 
Table 1

Means (M), Standard Deviations (SD), and Correlations of the Study Variables

\begin{tabular}{|c|c|c|c|c|c|c|c|c|}
\hline Variable & $M$ & $S D$ & 1 & 2 & 3 & 4 & 5 & 6 \\
\hline 1 ToLT & 3.37 & 1.81 & & $.71 * * *$ & $-.28 * * *$ & $.35 * * *$ & $-.10 *$ & \\
\hline 2 ToPLA & 3.27 & 1.97 & $.87 * * *$ & & $-.30 * * *$ & $.28 * * *$ & $-.13 * *$ & \\
\hline 3 On-task thoughts & 7.54 & 1.46 & $-.39 * * *$ & $-.26^{*}$ & & $-.41 * * *$ & $.36 * * *$ & \\
\hline 4 Thoughts of other things & 3.88 & 1.93 & $.45^{* * *}$ & $.39 * * *$ & $-.38 * * *$ & & $-.11 *$ & \\
\hline 5 Work engagement & 3.03 & 0.76 & -.16 & -.12 & $.41 * * *$ & -.18 & & \\
\hline 6 Pleasant anticipation $^{\mathrm{a}}$ & 3.97 & 0.85 & .23 & $.30 *$ & .00 & .00 & .04 & \\
\hline 7 Leisure plan valence & 5.98 & 1.11 & .01 & .02 & .14 & -.11 & $.35^{* *}$ & $.64 * * *$ \\
\hline
\end{tabular}

Note. ToLT $=$ Thoughts of leisure time. ToPLA $=$ Thoughts of a planned leisure activity. Correlations below the diagonal are person-level (between-person) correlations $(N=59-89)$ and correlations above the diagonal are hour-level (within-person) correlations of the person-mean centered variables $(N=438)$.

a of a planned leisure activity.

${ }^{*} p<.05 .{ }^{* *} p<.01 .{ }^{* * *} p<.001$.

\section{Planned Leisure Activities}

To further investigate the planned leisure activities, we differentiated between low-effort activities, physical activities, social activities, and household/childcare activities (cf. Sonnentag, 2001; ten Brummelhuis \& Bakker, 2012a). Two research assistants classified employees' planned leisure activities (Cohen's kappa $=.89)$. Employees engaged in low-effort activities (32.7\%), physical activities (30.8\%), social activities (21.1\%), and household/childcare activities (15.4\%). We used a univariate ANOVA to check for differences in pleasant anticipation and leisure plan valence. The results showed that the type of leisure activity matters for pleasant anticipation, $F(3,47)=4.32, p=.009$, partial $\eta^{2}=.22$, and leisure plan valence, $F(3,47)=5.87, p=.002$, partial $\eta^{2}=.27$. Post-hoc tests revealed that pleasant anticipation and leisure plan valence were significantly lower for household/childcare activities than for social, low-effort or physical activities. However, even for household/childcare activities, participants reported experiencing pleasant anticipation $(M=3.06, S D=1.22)$. Appendix A shows a list of all planned leisure activities and the associated pleasant anticipation and leisure plan valence.

The questionnaire in the next morning revealed that six employees did not engage in their planned leisure activity, yet, only three employees changed their leisure plan before 5:00 p.m. As the content of ToPLA may also change when the leisure plan changes, we excluded all questionnaires that were filled out after the leisure plan had changed (three hourly questionnaires). 


\section{Time Trend for Leisure Thoughts and their Relationship with Pleasant Anticipation}

In Hypothesis 1, we assumed a U-shaped time trend for leisure thoughts. Intra-class correlation coefficient $(1,1)$ for all thoughts indicated that it was appropriate to use multilevel modeling (.50 for ToLT, .57 for ToPLA, .30 for on-task thoughts, and .44 for thoughts of other things). We modelled the time trend by adding a linear and quadratic time variable and used polynomic terms to avoid multicollinearity (Bliese \& Ployhart, 2002). The results for all four types of thoughts are presented in Table 2. We found a significant quadratic time trend for ToLT $(\gamma=7.96, S E=1.57, p<.001)$ and ToPLA $(\gamma=8.77, S E=1.57, p<.001)$. For ToPLA, the linear time trend was also significant $(\gamma=5.48, S E=1.63, p<.001)$. The time trends for all types of thoughts is plotted in Figure 2 indicating our expected U-shaped time trend for ToLT and ToPLA. Thus, Hypothesis 1 was confirmed.

In Hypothesis 2, we expected that pleasant anticipation is positively related to the frequency of ToPLA. We found a significant positive relationship between pleasant anticipation and ToPLA between persons $(\gamma=1.06, S E=0.41, p=.013$; see Table 2$)$. Hence, Hypothesis 2 was supported.

\section{Figure 2}

Relationship Between the Frequency of Different Types of Thoughts and Time of the Working Day

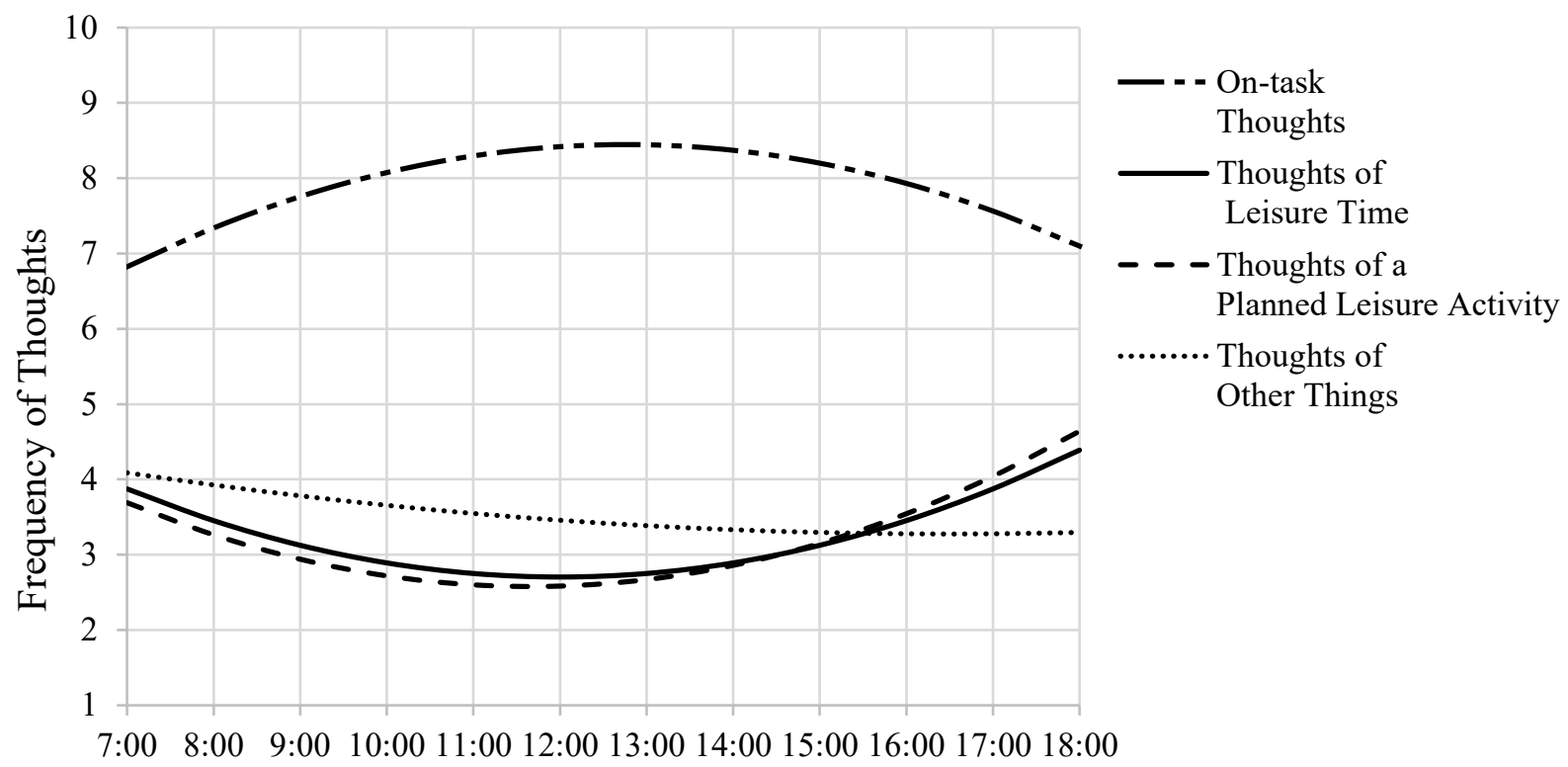

Time within the Working Day 


\section{Table 2}

Multilevel Regression Analyses to Predict Different Types of Thoughts with Time of the Working Day (Within-Person) and Pleasant Anticipation of a Planned Leisure Activity/Leisure Plan Valence (Between-Person)

\begin{tabular}{|c|c|c|c|c|c|c|c|c|c|c|c|c|}
\hline \multirow{2}{*}{ Variable } & \multicolumn{3}{|c|}{ ToLT } & \multicolumn{3}{|c|}{ ToPLA } & \multicolumn{3}{|c|}{ On-task thoughts } & \multicolumn{3}{|c|}{ Thoughts of other things } \\
\hline & Est & SE & $t$ & Est & $\overline{\mathrm{SE}}$ & $t$ & Est & $\mathrm{SE}$ & $t$ & Est & SE & $t$ \\
\hline Intercept & $3.12 * * *$ & 0.18 & 17.21 & $3.05 * * *$ & 0.21 & 14.81 & $7.99 * * *$ & 0.15 & 54.29 & $3.53 * * *$ & 0.19 & 18.18 \\
\hline Linear time trend & 2.87 & 1.64 & 1.76 & $5.48 * * *$ & 1.63 & 3.35 & 1.39 & 1.81 & 0.77 & $-4.19^{*}$ & 1.97 & -2.12 \\
\hline Quadratic time trend & $7.96 * * *$ & 1.57 & 5.06 & $8.77 * * *$ & 1.57 & 5.59 & $-8.40 * * *$ & 1.75 & -4.79 & 1.62 & 1.90 & 0.85 \\
\hline BIC & \multicolumn{3}{|c|}{1762.42} & \multicolumn{3}{|c|}{1769.50} & \multicolumn{3}{|c|}{1813.29} & \multicolumn{2}{|c|}{1908.52} & \\
\hline AIC & \multicolumn{2}{|c|}{1742.04} & & \multicolumn{2}{|c|}{1759.13} & & \multicolumn{2}{|c|}{1792.91} & & \multicolumn{2}{|c|}{1888.14} & \\
\hline$-2 \times \mathrm{LL}$ & \multicolumn{2}{|c|}{1732.04} & & \multicolumn{2}{|c|}{1749.13} & & \multicolumn{2}{|c|}{1782.91} & & \multicolumn{2}{|c|}{1878.14} & \\
\hline Pseudo $R^{2}$ & \multicolumn{2}{|c|}{0.03} & & \multicolumn{2}{|c|}{0.03} & & \multicolumn{2}{|c|}{0.04} & & \multicolumn{2}{|c|}{0.01} & \\
\hline Intercept & $3.02 * *$ & 0.23 & 13.00 & $3.04 * *$ & 0.26 & 11.74 & $8.31 * *$ & 0.16 & 51.21 & $3.54 * *$ & 0.24 & 14.46 \\
\hline Linear time trend & -0.11 & 2.00 & -0.06 & 1.15 & 1.93 & 0.60 & 3.91 & 2.27 & 1.72 & $-6.69^{*}$ & 2.59 & -2.56 \\
\hline Quadratic time trend & $9.80^{* *}$ & 1.85 & 5.31 & $13.11 * * *$ & 1.78 & 7.37 & $-9.19 * * *$ & 2.13 & -4.31 & 3.56 & 2.40 & 1.48 \\
\hline Pleasant anticipation ${ }^{a}$ & 0.74 & 0.37 & -1.05 & $1.06^{*}$ & 0.41 & 2.56 & -0.25 & 0.27 & -0.94 & 0.22 & 0.40 & 0.54 \\
\hline Leisure plan valence & -0.30 & 0.28 & -1.99 & -0.38 & 0.31 & -1.21 & $0.44^{*}$ & 0.21 & 2.15 & -0.34 & 0.30 & -1.13 \\
\hline $\mathrm{BIC}$ & \multicolumn{3}{|c|}{1118.17} & \multicolumn{3}{|c|}{1111.97} & \multicolumn{2}{|c|}{1152.95} & & \multicolumn{2}{|c|}{1243.27} & \\
\hline AIC & \multicolumn{2}{|c|}{1092.88} & & \multicolumn{2}{|c|}{1086.68} & & \multicolumn{2}{|c|}{1128.66} & & \multicolumn{2}{|c|}{1217.98} & \\
\hline$-2 \times \mathrm{LL}$ & \multicolumn{2}{|c|}{1078.88} & & \multicolumn{2}{|c|}{1072.68} & & \multicolumn{2}{|c|}{1114.66} & & \multicolumn{2}{|c|}{1203.98} & \\
\hline Pseudo $R^{2}$ & \multicolumn{2}{|c|}{0.02} & & $0 . C$ & & & 0 . & & & -0. & & \\
\hline
\end{tabular}

Notes. $N=438$ (upper part) and $N=279$ (lower part). ToLT $=$ Thoughts of leisure time. ToPLA = Thoughts of a planned leisure activity.

Estimates are unstandardized estimates. BIC $=$ Bayesian Information Criterion; AIC = Akaike's Information Criterion.

${ }^{\mathrm{a}}$ of a planned leisure activity $(N=59)$.

${ }^{*} p<.05 .{ }^{* *} p<.01 .{ }^{* * *} p<.001$. 


\section{Negative Relationship between Leisure Thoughts and Work Engagement}

In Hypotheses $3 \mathrm{a}$ und $3 \mathrm{~b}$ we focused on the relationship between ToLT/ToPLA and work engagement on the day-level (between-persons) and within one hour (concurrently). Before entering the predictors, we estimated a null-model (Raudenbush \& Bryk, 2002) and added a time trend for work engagement. The intra-class correlation coefficient for work engagement $(1,1)$ was .63 and we found a significant negative quadratic time trend $(\gamma=-1.26, S E=0.56$, $p=.025)$. A visual analysis of the scatterplot indicated that work engagement was higher during the middle of the working day and lower at the beginning and end of the working day. For all further analyses we used a model with fixed slopes and without autocorrelation or heterogeneity in the error structures due to the best fit to the data.

The results for ToLT/ToPLA and work engagement are presented in Table 3. For ToLT we found a significant negative relationship with work engagement between persons $(\gamma=-0.09$, $S E=0.04, p=.038)$, but not within persons $(\gamma=-0.03, S E=0.02, p=.186)$. For ToPLA the results did not reveal a significant negative relationship with work engagement between persons $(\gamma=-0.05, S E=0.04, p=.184)$, but within persons $(\gamma=-0.04, S E=0.02, p=.042)$. Hence, Hypothesis 3 was only partly supported. As we added an additional item for absorption, we repeated our analysis for each of the three sub-dimensions of work engagement. Yet, only for vigor, the relationships were significant for ToLT between persons $(\gamma=-0.11, S E=0.04$, $p=.009)$, ToPLA within persons $(\gamma=-0.05, S E=0.02, p=.017)$, and, additionally and in contrast to Table 3, for ToPLA between persons $(\gamma=-0.08, S E=0.04, p=.047) .^{5}$

\section{Relationship between ToPLA and Work Engagement for Different Levels of Leisure Plan}

\section{Valence}

In Hypothesis 4, we assumed that the relationship between ToPLA and work engagement in the subsequent hour (lagged) is positive/negative for a positive/negative leisure plan valence ${ }^{6}$. As we had to match two consecutive hours and only included participants who indicated a planned leisure activity, the number of measurement points was small compared to the test of Hypothesis $3 \mathrm{a} / 3 \mathrm{~b}$. As recommended, we grand-mean centered the level-2 moderator and used a random slope for ToPLA when testing for cross-level interaction (Aguinis et al., 2013).

\footnotetext{
${ }^{5}$ We also tested different models with the other types of thoughts as control variables. In sum, when adding ontask thoughts and one (or more) other types of thoughts, only on-task thoughts were significantly positively related to work engagement (between-person and within-person). In models without on-task thoughts and two or three other types of thoughts no significant relationships between any type of thoughts and work engagement were found.

${ }^{6}$ We thank the editor for the helpful suggestion on additional analyses.
} 
Table 3

Multilevel Regression Analyses Predicting Work Engagement

\begin{tabular}{|c|c|c|c|c|c|c|c|c|c|c|c|c|}
\hline \multirow{2}{*}{ Variable } & \multicolumn{3}{|c|}{ Model 1 (intercept only) } & \multicolumn{3}{|c|}{ Model 2 (time trend) } & \multicolumn{3}{|c|}{ Model 3a (ToLT) } & \multicolumn{3}{|c|}{ Model 3b (ToPLA) } \\
\hline & Est & $\mathrm{SE}$ & $t$ & Est & $\mathrm{SE}$ & $t$ & Est & $\mathrm{Se}$ & $t$ & Est & $\mathrm{Se}$ & $t$ \\
\hline Intercept & $3.03 * * *$ & 0.08 & 38.49 & $3.03 * * *$ & 0.08 & 38.61 & $3.05 * * *$ & 0.77 & 39.52 & $3.04 * * *$ & 0.78 & 38.78 \\
\hline Linear time trend & & & & 0.19 & 0.59 & 0.32 & 0.25 & 0.59 & 0.43 & 0.41 & 0.59 & 0.68 \\
\hline Quadratic time trend & & & & $-1.26^{*}$ & 0.56 & -2.25 & -1.04 & 0.58 & -1.79 & -0.92 & 0.58 & -1.57 \\
\hline Thoughts within persons & & & & & & & -0.03 & 0.02 & -1.33 & $-0.04 *$ & 0.02 & -2.03 \\
\hline Thoughts between persons ${ }^{a}$ & & & & & & & $-0.09 *$ & 0.04 & -2.11 & -0.05 & 0.04 & -1.34 \\
\hline $\mathrm{BIC}$ & \multicolumn{3}{|c|}{890.89} & \multicolumn{3}{|c|}{896.42} & \multicolumn{3}{|c|}{912.97} & \multicolumn{3}{|c|}{913.34} \\
\hline AIC & \multicolumn{3}{|c|}{878.65} & \multicolumn{3}{|c|}{876.04} & \multicolumn{3}{|c|}{884.48} & \multicolumn{3}{|c|}{884.84} \\
\hline$-2 \times \mathrm{LL}$ & \multirow{2}{*}{\multicolumn{3}{|c|}{872.65}} & \multicolumn{3}{|c|}{866.04} & \multicolumn{2}{|c|}{870.48} & & \multicolumn{3}{|c|}{870.84} \\
\hline Pseudo $R^{2}$ & & & & \multicolumn{3}{|c|}{0.01} & \multicolumn{3}{|c|}{0.04} & \multicolumn{3}{|c|}{0.02} \\
\hline
\end{tabular}

Notes. $N=438$ (within-person) and $N=89$ (between-person). ToLT $=$ Thoughts of leisure time. ToPLA = Thoughts of a planned leisure activity. Estimates are unstandardized estimates. BIC $=$ Bayesian Information Criterion; AIC $=$ Akaike's Information Criterion.

$* p<.05 . * * p<.01 . * * p<.001$. 
Before testing the moderation, we specified a model with ToPLA and leisure plan valence (see Table 4, Model 3). The results indicated that ToPLA was neither significantly negatively related to work engagement in the subsequent hour between persons $(\gamma=-0.08, S E=0.05, p=.165)$ nor within persons $(\gamma=-0.04, S E=0.03, p=.123)$. However, leisure plan valence was positively related to work engagement $(\gamma=0.20, S E=0.10, p=.044)$. In the next step, we added the interaction between ToPLA and leisure plan valence. The result revealed a significant interaction, $\gamma=-0.06, S E=0.03, p=.042^{7}$. To facilitate interpretation, the interaction is depicted in Figure 3. We used the $25^{\text {th }}$ percentile (leisure plan valence $=5.25$ ), the mean (leisure plan valence $=6.00$ ), and the $75^{\text {th }}$ percentile (leisure plan valence $=7.00$ ) for plotting the interaction. To analyse the simple slopes, we referred to an online tool presented by Preacher, Curran, and Bauer (2006). Contrary to our prediction, the relationship between ToPLA and work engagement was negative for a positive leisure valence, $b=-0.10, S D=0.04, t=-2.69, p=.010$. Regarding a negative leisure plan valence, we could not test the relationship between ToPLA and work engagement, as the $25^{\text {th }}$ percentile of the leisure plan valence was still positive. Yet, the test of the simple slope at the $25^{\text {th }}$ percentile revealed that for employees with a slightly positive leisure plan valence the relationship was non-significant, $b=0.00, S D=0.04, t=0.10, p=$ $.918^{8}$. Thus, Hypothesis 4 was rejected. Again, we analysed the data with the sub-dimensions of work engagement. The interaction term was significant for absorption $(\gamma=-0.07, S E=0.03$, $p=.041)$, yet not for dedication $(\gamma=-0.06, S E=0.04, p=.089)$ or vigor $(\gamma=-0.03, S E=0.03$, $p=.445){ }^{9}$

\section{Additional Analysis}

Although we assumed that ToPLA distract employees within one hour and the content of ToPLA therefore may not matter, we tested whether leisure plan valence or pleasant anticipation of the planned leisure activity moderates the relationship between ToPLA and work engagement within the same hour. The results showed that the interaction was neither significant for pleasant anticipation $\left(\gamma=-0.02, S E=0.04, p=.60\right.$, Pseudo $\left.R^{2}=.06\right)$ nor for leisure plan valence $\left(\gamma=0.00, S E=0.03, p=.880\right.$, Pseudo $\left.R^{2}=.10\right)$.

\footnotetext{
${ }^{7}$ By contrast, pleasant anticipation did not moderate the relationship between ToPLA and work engagement $\left(\gamma=-0.06, S E=0.04, p=.090\right.$, Pseudo $\left.R^{2}=.02\right)$.

${ }^{8}$ Although only eight participants met the criteria for the $10^{\text {th }}$ percentile indicating neutral and negative leisure plan valence ratings $(<5.00)$, we tested the simple slope, $b=0.08, S D=0.06, t=1.196, p=.234$.

${ }^{9}$ When adding one or more other types of thoughts as control variables, the interaction term remained significant. Independently of the other types of thoughts, on-task thoughts within persons were significantly related to higher work engagement in the subsequent hour.
} 


\section{Table 4}

Multilevel Regression Analyses Predicting Work Engagement in the Subsequent Hour (lagged)

\begin{tabular}{|c|c|c|c|c|c|c|c|c|c|c|c|c|}
\hline \multirow{2}{*}{ Variable } & \multicolumn{3}{|c|}{ Model 1 (intercept only) } & \multicolumn{3}{|c|}{ Model 2 (time trend) } & \multicolumn{3}{|c|}{ Model 3 (ToPLA) } & \multicolumn{3}{|c|}{ Model 4 (ToPLA) } \\
\hline & Est & $\mathrm{SE}$ & $t$ & Est & SE & $t$ & Est & $\mathrm{Se}$ & $t$ & Est & $\mathrm{Se}$ & $t$ \\
\hline Intercept & $3.15 * * *$ & 0.11 & 28.39 & $3.13 * * *$ & 0.11 & 28.12 & $3.14 * * *$ & 0.11 & 29.18 & $3.13 * * *$ & 0.11 & 28.99 \\
\hline Linear time trend & & & & $-3.27 *$ & 1.50 & -2.18 & $-2.96^{*}$ & 1.50 & -1.50 & -2.76 & 1.50 & -1.84 \\
\hline Quadratic time trend & & & & -0.98 & 1.49 & -0.67 & 0.10 & 1.56 & 0.07 & -0.11 & 1.54 & -0.72 \\
\hline Thoughts within persons & & & & & & & -0.04 & 0.03 & -1.55 & -0.04 & 0.03 & -1.47 \\
\hline Thoughts between persons & & & & & & & -0.08 & 0.05 & -1.41 & -0.07 & 0.05 & -1.22 \\
\hline Leisure plan valence & & & & & & & $0.20^{*}$ & 0.10 & 2.07 & 0.19 & 0.10 & 1.90 \\
\hline Thoughts within persons*Valence ${ }^{a}$ & & & & & & & & & & $-0.06^{*}$ & 0.03 & -2.05 \\
\hline $\mathrm{BIC}$ & \multicolumn{2}{|c|}{367.37} & & \multicolumn{2}{|c|}{367.03} & & \multicolumn{2}{|c|}{396.53} & & \multicolumn{2}{|c|}{403.75} & \\
\hline AIC & \multicolumn{2}{|c|}{357.68} & & \multicolumn{2}{|c|}{350.93} & & \multicolumn{2}{|c|}{363.49} & & \multicolumn{2}{|c|}{368.57} & \\
\hline$-2 \times \mathrm{LL}$ & \multirow{2}{*}{\multicolumn{2}{|c|}{351.68}} & & \multicolumn{2}{|c|}{340.93} & & \multicolumn{2}{|c|}{344.49} & & \multicolumn{2}{|c|}{346.57} & \\
\hline Pseudo $R^{2}$ & & & & \multicolumn{2}{|c|}{0.02} & & \multicolumn{2}{|c|}{0.08} & & \multicolumn{2}{|c|}{0.09} & \\
\hline
\end{tabular}

Notes. $N=188$ (within-person) and $N=51$ (between-person). ToPLA = Thoughts of a planned leisure activity. Estimates are unstandardized estimates. BIC = Bayesian Information Criterion; AIC = Akaike's Information Criterion. Estimates are unstandardized estimates.

${ }^{\mathrm{a}}$ Leisure plan valence.

${ }^{*} p<.05 .{ }^{* *} p<.01 .{ }^{* * *} p<.001$. 
Figure 3

Relationship Between Thoughts of a Planned Leisure Activity and Work Engagement in the Subsequent Hour for Different Leisure Plan Valence Ratings

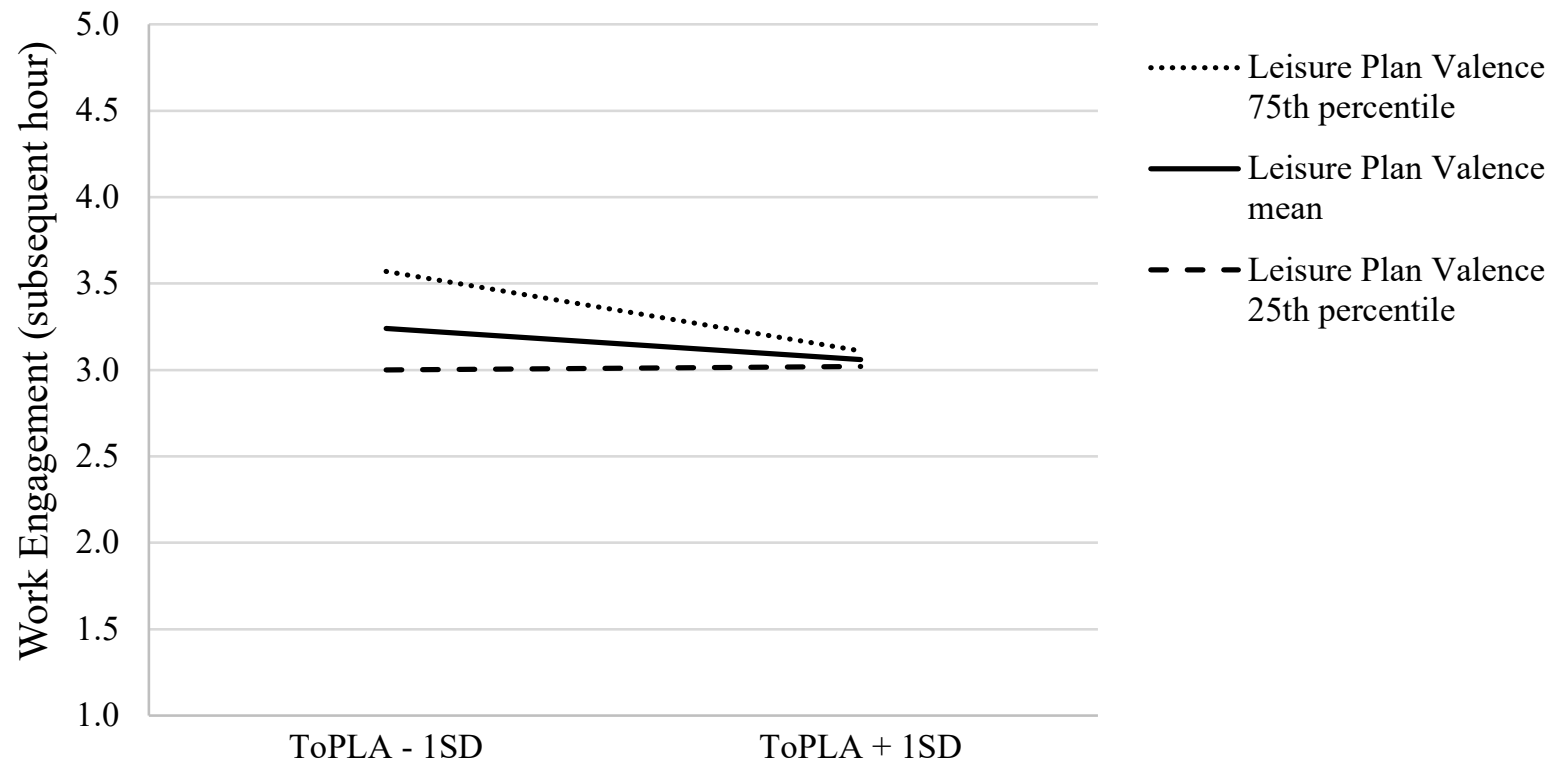

Frequency of Thoughts of a Planned Leisure Activity (ToPLA)

Note. With regard to the bipolar scale $(1=$ very negative; $4=$ neutral; $7=$ very positive $)$ and the skewed distribution of leisure plan valence, the $25^{\text {th }}$ percentile already includes leisure plans above a positive valence rating (leisure plan valence $>5.25$ ), and leisure plans at the $75^{\text {th }}$ percentile are very positive (leisure plan valence $=7$ ).

As both job and personal resources are important for employees' work engagement (Bakker \& Demerouti, 2017; Xanthopoulou et al., 2007), we tested our Hypotheses 3a/3b and Hypothesis 4 with different level-2 control variables. For job resources, we controlled for autonomy by including task control and time control each assessed with three items (Semmer et al., 1999). For ToLT, the significant between-person relationship with work engagement disappeared $\left(\gamma=-0.07, S E=0.05, p=.117\right.$, Pseudo $\left.R^{2}=0.08\right)$. For ToPLA, the within-person relationship with work engagement was still significant $(\gamma=-0.04, S E=0.02, p=.041$, Pseudo $R^{2}=0.06$ ). The cross-level interaction (Hypothesis 4) changed slightly and became nonsignificant $\left(\gamma=-0.07, S E=0.03, p=.071\right.$, Pseudo $\left.R^{2}=0.11\right)$. Regarding personal resources, we measured employees' self-regulation, which is closely related to general self-efficacy, with five items (Diehl et al., 2006). The results showed the same pattern as for job resources: for ToLT, the significant between-person relationship with work engagement disappeared $(\gamma=-$ $0.04, S E=0.04, p=.408$, Pseudo $R^{2}=0.12$ ). For ToPLA, the within-person relationship with work engagement was still significant $\left(\gamma=-0.04, S E=0.02, p=.043\right.$, Pseudo $\left.R^{2}=0.12\right)$. 
Again, the cross-level interaction (Hypothesis 4) changed slightly and became non-significant $\left(\gamma=-0.05, S E=0.03, p=.090\right.$, Pseudo $\left.R^{2}=0.18\right)$.

We further tested for reverse causation to ascertain whether high work engagement was an antecedent of ToLT/ToPLA in the subsequent hour. We found a non-significant relationship between work engagement and ToLT in the subsequent hour within persons $(\gamma=0.27$, $S E=0.20, p=.193)$ and a significant negative relationship between persons $(\gamma=-0.66$, $S E=0.28, p=.021)$. Pseudo $R^{2}$ was .00 . Moreover, work engagement was not significantly related to ToPLA in the subsequent hour neither within persons $(\gamma=0.22, S E=0.21, p=.306)$ nor between persons $(\gamma=-0.37, S E=0.33, p=.264)$. Pseudo $R^{2}$ was .00 .

\section{Discussion}

The present study aimed to shed light on employees' leisure thoughts during the working day. In line with our assumptions, we found that employees reported ToLT and ToPLA, which concurs with research on future-oriented thoughts (Barsics et al., 2016; D’Argembeau, 2018) Regarding the frequency of ToLT/ToPLA, our results revealed that employees reported more ToLT/ToPLA at the beginning and at the end of the working day (U-shaped time trend), which is in line with construal level theory (Liberman \& Trope, 1998; Wiesenfeld et al., 2017) and showed that construal level theory is appropriate to predict time trends. For thoughts of other things, we only found a negative linear time trend, which supports the idea that leisure thoughts can be differentiated from other off-task thoughts. Regarding on-task thoughts, the inverted Ushaped time trend was inverse to the U-shaped time trend of ToLT/ToPLA. Employees were thus less focused on their work if they reported leisure thoughts and vice versa. These results underpin the assumption that employees are not always focused on their work (Beal et al., 2005; Merlo et al., 2018).

Besides, we found a linear time trend for ToPLA, but not for ToLT. Although employees reported both more ToLT/ToPLA at the end of the working day than in the middle of the working day (U-shaped time trend), the additional linear time trend for ToPLA indicates that employees thought more of their planned leisure activity at the end of the working day. Hence, a differentiation between ToPLA and ToLT seems reasonable.

The second aim of the study was to investigate whether high pleasant anticipation of the planned leisure activity enhances the frequency of ToPLA across the working day. The results revealed that employees reported more ToPLA if they experienced high pleasant anticipation than did employees experiencing low pleasant anticipation. As expected, pleasant anticipation did not increase the frequency of ToLT, as ToLT are not related to a specific, planned leisure 
activity. The differentiation between ToLT and ToPLA is necessary and shows that employees not only think of their leisure time in general but also of specific, planned leisure activities. Our analyses also showed that only pleasant anticipation of the planned leisure activity was a valid predictor of ToPLA, whereas leisure plan valence did not predict ToPLA. Thus, the differentiation between pleasant anticipation and leisure plan valence is important.

The third aim of the study was to investigate the relationship between ToLT/ToPLA and employees' work engagement across the working day, within an hour, and in the subsequent hour. Across the working day, employees with a higher frequency of ToLT reported lower work engagement than did employees with a lower frequency of ToLT. Furthermore, employees indicated lower work engagement during hours with a high frequency of ToPLA compared to hours with low frequency of ToPLA. Although these results were mixed, they foster the conception of ToLT/ToPLA as off-task thoughts expected to have negative consequences for performance (e.g., Randall et al., 2014). When employees generated leisure thoughts either within one hour or across the working day, they were not able to focus intensively on their work.

In contrast to the negative relationship between ToLT/ToPLA an work engagement within the same hour, we expected that ToPLA is positively related to work engagement in the subsequent hour when leisure plan valence is positive. We also assumed that when leisure plan valence is negative, the relationship between ToPLA and work engagement in the subsequent hour is negative. The results showed that leisure plan valence moderates the relationship between ToPLA and work engagement in the subsequent hour. Yet, in contrast to our expectations, the relationship was negative for a positive leisure plan valence. When two employees reported a very positive leisure plan valence, the employee with the higher frequency of ToPLA within the one hour is expected to show lower work engagement in the subsequent hour compared to the employee with the lower frequency of ToPLA. Thus, thinking frequently of a leisure activity with a very positive leisure plan valence decreases work engagement in the subsequent hour.

When employees think of a leisure plan with a positive leisure plan valence, they may stick to these positive thoughts. According to fantasy realization theory (e.g., Oettingen et al., 2001; Oettingen, 2012), just thinking about a positive future will neither change behaviour nor cognition, as individuals indulge in the positive future. As a consequence, they forget about the obstacles, which they have to overcome to achieve their future goals and they are not motivated to invest resources in changing the present. When employees generate ToPLA with a positive leisure plan valence, they may not recognize other tasks (i.e., obstacles) they have to finish 
before they can engage in their planned leisure activity. Therefore, they may not know where to invest resources and cannot become engaged. One may also speculate that thoughts of a positive leisure activity motivate employees to savour the positive future event not only in the present but also in the subsequent hour and/or to start detaching from work.

The result that frequently thinking of positive planned leisure activity is negatively related to work engagement in the subsequent hour also adds new insight to a diary study by Dumas and Perry-Smith (2018) on the interaction of family structure, planned leisure activities, and absorption. The authors found that for single and childless employees the proportion of domestic leisure activities (e.g. household, childcare) to other leisure activities (e.g., physical activities, meeting with friends) was lower and that these employees also reported lower absorption. Dumas and Perry-Smith (2018) assumed that planned domestic leisure activities reinforce a goal-directed mindset, while other planned leisure activities distract employees from their work. Although we chose another approach and referred to leisure plan valence, our results concur with their research. The interaction between ToPLA and leisure plan valence showed that when employees reported a positive leisure plan valence (which did not apply to domestic leisure activities, see Appendix A) and more ToPLA, their work engagement was lower in the subsequent hour. Frequently thinking of a positive leisure activity may not only distract employees from their task at hand but may also change their mindset from a goal-directed mindset to an open (i.e., leisure-oriented) mindset hindering employees from becoming engaged in the subsequent hour. Our research provides evidence that thoughts may explain why a lower proportion of domestic leisure activities to other leisure activities was related to lower absorption (Dumas \& Perry-Smith, 2018).

For a negative leisure plan valence (i.e., a resource loss), we could not test the relationship between ToPLA and work engagement in the subsequent hour, as only eight participants indicated a neutral or negative leisure plan valence. However, when employees reported a low positive leisure plan valence (i.e., one point above neutral), the relationship between ToPLA and work engagement in the subsequent hour was non-significant. Thus, when employees' leisure plan valance was only slightly positive, it did not matter for their subsequent hour's work engagement whether they frequently thought about their planned leisure activity (high vs. low ToPLA). It may be that under these circumstances ToPLA neither activate a goal-directed mindset nor activate indulging in the future. However, as the distribution of leisure plan valence was skewed, we could not answer the question in which way a negative leisure plan valence would have changed the relationship between ToPLA and work engagement in the subsequent hour. 
It is important to note that the results for leisure plan valence exist due to a cross-level interaction, which accounts for hourly ToPLA and the day-level leisure plan valence. As the between-person relationship between leisure plan valence and work engagement was positive, it would be misleading to state that positive planned leisure activities are in general negatively related to work engagement. We therefore still suggest that thinking of a positive planned leisure activity may indicate an upcoming resource gain. There are several explanations why we failed to find a positive relationship between ToPLA and work engagement for employees with a positive leisure plan valence. First, it is possible that such a relationship does not exist and ToPLA are always negatively related with work engagement. Thus, a positive leisure plan valence is only beneficial for work engagement when employees did not think of the planned leisure activity.

Second, it might not only be the frequency, but also the content of ToPLA that must be understood in more detail. As we did not ask participants to report the specific content of their thoughts in order to distract them as little as possible, we did not know whether employees' thoughts about their planned positive leisure activities were positive themselves. For instance, rating an anticipated park walk as a positive leisure plan might enhance work engagement across the working day (e.g., high leisure plan valence). However, this could be tainted by negative ToPLA about the planned activity when the weather is cloudy. Therefore, the effects on work engagement may have disappeared.

Third, we asked participants to report a planned leisure activity with only few words and did not ask for a detailed description of their plans. However, their plans might have ranged from very detailed step-by-step plans to simple plans for the future leisure activity. As for detailed plans the realization of a plan becomes more likely (e.g., Gollwitzer, 1996; Schmitt et al., 2019), it might be that for employees with an elaborated leisure plan the anticipated resource gain is more salient. Thus, ToPLA may only positively relate to work engagement in the subsequent hour when leisure plan valence is positive and the leisure plan itself is well elaborated.

Finally, it is also conceivable that when task complexity is low, ToPLA, in combination with a positive leisure plan valence, may positively relate to work engagement in the subsequent hour. During the working day, tasks differ in their complexity (Beal et al., 2005) and interruptions (e.g., ToPLA) impair the performance of highly complex tasks while enhancing the performance for simpler tasks (Speier et al., 1999). Furthermore, the negative influence of off-task thoughts on performance decreases if the task is less complex (Randall et al., 2014). 
This would be in line with Dane (2018), who assumed that off-task thoughts are less problematic if the need for monitoring is low.

\section{Theoretical and Practical Implications}

Our study contributes to the research on attentional focus (Beal et al., 2005; Leroy, 2009; Merlo et al., 2018) by introducing the concept of leisure thoughts as a special type of off-task thoughts. Leisure thoughts followed a quadratic time trend and the frequency of ToPLA was related to pleasant anticipation. Our research thus demonstrated that leisure thoughts are different from more general off-task thoughts (i.e., thoughts of other things), and that different types of offtask thoughts have different time trends. Moreover, the time trend for leisure thoughts provided additional evidence for psychological reattachment (Sonnentag et al., 2019; Sonnentag \& Kühnel, 2016) and detachment (Sonnentag \& Bayer, 2005; Sonnentag \& Kruel, 2006), as we investigated thoughts that may relate to these two processes. On the one hand, employees had more leisure thoughts at the beginning of the working day, which indicates their need to reattach to work. On the other hand, employees generated more leisure thoughts at the end of the working day, which implies that they start to detach from work. The time trend for on-task thoughts further confirmed this interpretation.

This is the first study to investigate pleasant anticipation of a planned leisure activity as part of a normal working day. In general, studies on pleasant anticipation examine long-lasting events like vacations (Nawijn et al., 2013; J. L. Smith \& Bryant, 2013), weekends (Sonnentag, Mojza, et al., 2008) or special events like Christmas (Bryant, 2003; Syrek, Weigelt, et al., 2018). Our research demonstrated that pleasant anticipation is also important for daily recurring events like leisure time. This is important; behaviour may be influenced more strongly by the anticipation of an upcoming event than by past experience (Baumeister et al., 2007). We therefore assume that anticipation is a neglected variable and recommend that researchers should pay more attention to anticipation as an explanation for behaviour.

Contrary to our expectations, we only found negative relationships between ToPLA and work engagement. Yet, leisure plan valence itself was positively associated with work engagement across the working day. When employees stated in the morning that their planned leisure activity will be positive, they were more engaged in their work across the working day compared to employees with less positive planned leisure activities. It seems to be important for employees to have a positive planned leisure activity in mind to become highly engaged in their work. Thus, not only engaging in leisure activities but also having a leisure plan in the morning may relate to employees' work engagement. However, employees should not think 
about their planned leisure activity frequently, as ToPLA distract them from their work within the same hour. Furthermore, when the planned leisure activity is very positive even the relationship between ToPLA and work engagement in the subsequent hour is negative. Thus, ToPLA are not only relevant for work engagement within the same hour but also in the subsequent hour. We suggest that employees should still plan a positive leisure activity and then allocate a fixed time (e.g., 10 minutes at the beginning of their working day) for leisure thoughts. This fixed time could become a ritual and may help employees to reduce leisure thoughts afterwards and to focus better on their work during the working day.

Finally, our analysis of the time trend for work engagement revealed that employees were most engaged in their work during the middle of their working day. This has some implications for employees' self-management, especially for their time planning (e.g., Parke et al., 2018). First, employees should use the beginning and the end of their working day for tasks that do not require a high level of engagement. For instance, employees could start the working day with phone calls and end the working day writing e-mails, thereby using their high level of work engagement during the middle of the day for more complex tasks. Second, employees could use lunch breaks to detach from their work, which is important for their concentration afterwards. As detachment is defined by not thinking of work-related issues (Sonnentag \& Bayer, 2005), employees could use ToLT/ToPLA to detach from their work during their lunch breaks. In turn, they may generate fewer leisure thoughts for the rest of the working day and be less prone to distractions.

\section{Limitations and Directions for Future Research}

Our study is not without limitations, and these provide indications for further research. First, we asked our participants to report their leisure thoughts in every hourly questionnaire and thereby caused them to think of their leisure time. The frequency of ToLT/ToPLA may therefore have been due to our measurement method. However, asking participants directly about their thoughts is a common method in research on the frequency of thoughts (Barsics et al., 2016; D'Argembeau et al., 2011). In addition, studies on rumination are conducted using similar methods and ask participants for their ruminative thoughts (e.g., Flaxman et al., 2018). Moreover, participants often skipped one or two hourly questionnaires, which increased the time between two measurement points. Even if there are effects due to our measurement method, we found the expected U-shaped time trend for leisure thoughts and not for thoughts of other things, which cannot be explained by the measurement method. In summary, we argue 
that the measurement method had only a very small influence on the frequency of leisure thoughts.

Second, we chose one single working day with hourly measurement points as we focused on hourly changes in ToLT/ToPLA and their relationship with work engagement. However, we cannot establish whether employees differ in their anticipations from day to day. Although we suggest that pleasant anticipation changes within persons, future research should measure pleasant anticipation at different days using a daily diary design.

Third, pleasant anticipation and leisure plan valence were highly correlated, although we found some empirical evidence that these constructs are different (i.e., they differ in their relationship with ToPLA). One explanation for the high correlation may be that participants did not use the full range of the valence scale. Future research may ask participants about leisure plan valence and pleasant anticipation of different hypothetical scenarios using our extensive definition of pleasant anticipation to manipulate features of these scenarios (e.g. probability of occurrence). This could help to investigate differences between leisure plan valence and pleasant anticipation, and to gain further insights under which conditions pleasant anticipation is experienced.

Fourth, only one participant named a leisure activity with a negative leisure plan valence. We therefore could not test whether the relationship between ToPLA and work engagement in the subsequent hour is different for negative compared to positive planned leisure activities. This relationship was negative for very positive planned leisure activities, yet we do not know what this relationship would look like for (very) negative leisure activities. To take a closer look at this question, participants could be asked to name a positive and/or a negative planned leisure activity instead for their planned leisure activity in general. Furthermore, some employees named more than one planned leisure activity. Thus, their estimation of leisure plan valence and/or pleasant anticipation could refer to one of these leisure activities or could reflect an overall rating of their leisure time.

Finally, we used self-reports in our study, which are a source of common method variance (Podsakoff et al., 2012). We had to rely on self-reports with respect to our focal construct, leisure thoughts, as thoughts are only accessible to individuals themselves. For work engagement, it was also necessary to use self-reports, since work engagement describes an internal personal state (Sonnentag et al., 2010). 


\section{Conclusion}

Our study introduced leisure thoughts as a new construct. We demonstrated that two types of leisure thoughts, thoughts of leisure time (ToLT) and thoughts of a planned leisure activity (ToPLA), can be differentiated. We also shed light on the occurrence of both types of leisure thoughts and found that these were more prevalent at the beginning and the end of the working day. Furthermore, pleasant anticipation of a planned leisure activity enhanced the frequency of thoughts of this leisure activity. We found that leisure thoughts relate negatively to work engagement within the same hour and across the working day. Contrary to our expectation, our results revealed that for a positive planned leisure activity the relationship between thoughts of this planned activity and work engagement in the subsequent hour was negative. However, having planned a positive leisure activity in the morning was positively related to work engagement across the working day. In sum, our study constitutes a first step to connect employees' upcoming leisure time with their present work engagement via the phenomenon of leisure thoughts.

\section{Acknowledgment}

We thank Antje Schmitt for her helpful comments and stimulating discussions on leisure thoughts. We thank Julia Köbler and Alexander Sadi for their help with data collection, and Caroline Becker, Isabella Hebel, and Lennart Pötz for conducting the interviews.

\section{Disclosure statement}

No potential conflict of interest was reported by the authors. 


\section{Appendix A}

Table 1

List of all Planned Leisure Activities and the Associated Pleasant Anticipation and Leisure Plan Valence.

\begin{tabular}{|c|c|c|}
\hline Planned leisure activity & $\begin{array}{c}\text { Pleasant } \\
\text { anticipation }^{\mathrm{a}}\end{array}$ & $\begin{array}{l}\text { Leisure plan } \\
\text { valence }^{\text {b }}\end{array}$ \\
\hline Paying building materials & 5.00 & 6 \\
\hline Refreshing / vitalizing & 5.00 & 7 \\
\hline Riding a bike & 5.00 & 7 \\
\hline $\begin{array}{l}\text { Meeting up witch family; doctor's } \\
\text { appointment; doing sports }\end{array}$ & 5.00 & 6 \\
\hline Meeting up with family & 5.00 & 7 \\
\hline Watching a movie & 5.00 & 7 \\
\hline Meeting up with friends & 5.00 & 7 \\
\hline Singing course & 5.00 & 7 \\
\hline Go riding a horse & 5.00 & 7 \\
\hline Go sleeping & 5.00 & 4 \\
\hline Watching a series & 5.00 & 7 \\
\hline Play something & 5.00 & 7 \\
\hline Doing sports & 5.00 & 7 \\
\hline Tai Chi course & 5.00 & 7 \\
\hline Watching TV & 4.75 & 6 \\
\hline Yoga course & 4.75 & 7 \\
\hline English theatre & 4.50 & 6 \\
\hline Meeting up with friends & 4.50 & 7 \\
\hline Ironing; riding a bike & 4.25 & 6 \\
\hline Visiting sauna & 4.25 & 6 \\
\hline Tiding out closet & 4.25 & 7 \\
\hline Go running for 2.5 hours & 4.00 & 6 \\
\hline Cooking dinner & 4.00 & 6 \\
\hline Going to the gym & 4.00 & 7 \\
\hline Relaxing & 4.00 & 4 \\
\hline Visiting friends & 4.00 & 5 \\
\hline Meeting up with friends & 4.00 & 7 \\
\hline Football training & 4.00 & 6 \\
\hline Householding; watching a movie & 4.00 & 6 \\
\hline Cooking with friends & 4.00 & 6 \\
\hline Physical therapy & 4.00 & 6 \\
\hline Back workout & 4.00 & 7 \\
\hline Having sex & 4.00 & 7 \\
\hline Doing sports; Zumba & 4.00 & 6 \\
\hline Meeting up with friends & 4.00 & 6 \\
\hline
\end{tabular}


Table 1

(continued)

\begin{tabular}{lcc}
\hline \multicolumn{1}{c}{ Planned leisure activity } & $\begin{array}{c}\text { Pleasant } \\
\text { anticipation }^{\mathrm{a}}\end{array}$ & $\begin{array}{c}\text { Leisure plan } \\
\text { valence }^{\mathrm{b}}\end{array}$ \\
\hline Planning a holiday & 4.00 & 6 \\
Go bowling & 3.75 & 7 \\
Go out for dinner and phone friends & 3.75 & 5 \\
Getting healthy from cold & 3.75 & 4 \\
Going out with the dog & 3.75 & 5 \\
Doing sports; swimming & 3.75 & 6 \\
Watching TV & 3.75 & 7 \\
Go running & 3.75 & 6 \\
Reading a book & 3.50 & 6 \\
Watching Netflix & 3.50 & 7 \\
Reading the newspaper & 3.50 & 6 \\
Listing to an audiobook & 3.25 & 7 \\
Reading; crafting & 3.25 & 5 \\
Coming down & 3.25 & 6 \\
Go swimming & 3.25 & 6 \\
Second job & 3.00 & 4 \\
Meeting up with friends & 2.75 & 4 \\
Pick up car & 2.50 & 3 \\
Watching TV & 2.50 & 5 \\
Watching football & 2.50 & 5 \\
Office work at German red cross & 2.00 & 4 \\
Doing the laundry; packing bags & 2.00 & 5 \\
Householding & 1.75 & 3 \\
\hline Note. N 59. & & \\
\hline
\end{tabular}

Note. $N=59$.

${ }^{\mathrm{a}}$ of the planned leisure activity.

${ }^{\mathrm{b}}$ single item ranging from 1 (very negative) to 7 (very positive). 


\title{
STUDY 3: A DIARY STUDY ON ANTICIPATED LEISURE TIME, MORNING RECOVERY, AND EMPLOYEES' WORK ENGAGEMENT
}

Sebastian Seibel ${ }^{a,{ }^{*}}$ and Judith Volmer ${ }^{a}$

${ }^{a}$ Work and Organizational Psychology Group, Otto-Friedrich University of Bamberg; Bamberg, Germany

${ }^{b}$ Work and Organizational Psychology Group, University of Wuerzburg; Wuerzburg, Germany

This is an Accepted Manuscript of an article published by MDPI under an open access Creative Commons CC BY 4.0 license and is available at: https://www.mdpi.com/1660-4601/18/18/9436

\begin{abstract}
Recovery during yesterday's leisure time is beneficial for morning recovery, and morning recovery fosters employees' work engagement, a positive, motivational state associated with job performance. We extended existing research by assuming that both, morning recovery (considered a resource) and anticipated leisure time (considered an anticipated resource gain), relate to work engagement. Anticipated leisure time comprises two constructs: general anticipation of leisure time, which refers to employees' cognitive evaluation of their entire upcoming leisure time, and pleasant anticipation of a planned leisure activity, which describes a positive affective reaction because of one specific, upcoming leisure activity. We suggest that employees with high pleasant anticipation generate more thoughts of a planned leisure activity (ToPLA), which may distract them from their work, reducing their work engagement. A diary study over five days showed that morning recovery and general anticipation of leisure time were positively related to work engagement. Furthermore, employees with higher pleasant anticipation of a planned leisure activity reported more ToPLA. In contrast to our expectations, neither pleasant anticipation nor ToPLA were related to work engagement. In sum, this study introduced anticipated leisure time as a novel antecedent of work engagement and demonstrated that anticipated resources gains are important for high work engagement.
\end{abstract}

Keywords: morning recovery; leisure time; pleasant anticipation; work engagement; thoughts of a planned leisure activity; diary study 


\section{Theoretical Background}

During the working day, employees must deal with many different job demands and stressors, which drain their resources and exhaust their energy (Meijman \& Mulder, 1998; Trougakos \& Hideg, 2009). To stay healthy, recovery after the working day is essential for employees (for a meta-analysis, see Steed et al., 2019), and research has shown that leisure activities are critical to a successful recovery process (for a review, see Demerouti et al., 2009). Recovered employees are less fatigue and more vigorous (for a meta-analysis, see Bennett et al., 2018) and report higher psychological well-being and job performance (Steed et al., 2019). One indicator of job performance is employees' work engagement (Christian et al., 2011; Schneider et al., 2018), a positive, motivational, work-related state (Schaufeli et al., 2006b). Work engagement is related to employees' job performance and health (for a meta-analysis, see Halbesleben, $2010 \mathrm{~b}$ ); therefore, it is relevant for organisations and employees.

Until now, the majority of studies on recovery processes and work engagement has focused on the relationship between yesterday's leisure time and present work engagement (e.g., Garrick et al., 2014; Sonnentag, 2003; ten Brummelhuis \& Bakker, 2012b). For instance, participating in social activities during leisure time (e.g., meeting with friends) helps employees become more engaged during the next working day (ten Brummelhuis \& Bakker, 2012b). However, besides the importance of past leisure time, thinking about upcoming leisure time is part of everyday thoughts (D'Argembeau et al., 2011; Seibel et al., 2020), and therefore, employees may also anticipate their upcoming leisure time while they are still at work. Previous research has demonstrated that anticipation is relevant for employees' behaviours, e.g., when employees anticipate high workload, they put more effort into approach coping (e.g., planning their workdays; Casper et al., 2017). Furthermore, anticipated time pressure has shown to have impaired task performance in several experiments (Leroy \& Glomb, 2018b). In sum, there is initial evidence that employees think about their leisure time and consider anticipated events. Thus, we extend the perspective of existent research on yesterday's leisure time and investigate whether the anticipation of upcoming leisure time also relates to work engagement.

In our study, we take a closer look at two different types of anticipation of leisure time. First, we examine the general anticipation of leisure time, which refers to an overall evaluation of employees' upcoming leisure time. Second, we investigate employees' pleasant anticipation of a planned leisure activity, reflecting a positive, affective reaction because of one specific upcoming leisure activity ${ }^{1}$. We draw on Conservation of Resources (COR) theory (Hobfoll,

\footnotetext{
${ }^{1}$ We use the term leisure activity to refer to all activities that take place during employees' leisure time.
} 
1989) and propose that both types of anticipation indicate an upcoming resource gain. According to COR theory (Hobfoll, 1989), not only present but also anticipated resource gains/losses are part of individuals' resource management (Gorgievski \& Hobfoll, 2008a; Hobfoll et al., 2003); therefore, we hypothesize that general anticipation and pleasant anticipation are positively related to work engagement. However, employees with higher pleasant anticipation of a planned leisure activity reported more thoughts of a planned leisure activity (ToPLA) during the working day (Seibel et al., 2020). ToPLA are a type of off-task thoughts (e.g., Beal et al., 2005), and research has shown that ToPLA are negatively related to work engagement (Seibel et al., 2020). Consequently, a negative relationship between pleasant anticipation of a planned leisure activity and work engagement, mediated by ToPLA, may also exist.

This study has three main research goals. First, we investigate whether the anticipation of upcoming leisure time and present work engagement are related. We thereby add to previous research on anticipation in the working context (Casper et al., 2017; DiStaso \& Shoss, 2020; Leroy \& Glomb, 2018b). Because the anticipation of upcoming events plays an important role in individuals' behaviour, feelings, and cognitions (e.g., Baumeister et al., 2016; Oettingen, 2012), and leisure time is a recursive part of employees' daily lives, the current research aims at investigating further precursors of employees' work engagement. Because work engagement has a substantial impact on employees' well-being and performance (Halbesleben, 2010b; Schneider et al., 2018), researchers are interested in identifying various antecedents of work engagement. These insights may point out new employee training programmes and add to existing recovery training (Hahn et al., 2011).

The second aim of our study is to shed light on whether widely investigated previous leisure time experiences and anticipated leisure time activities are jointly associated with work engagement. Therefore, we refer to morning recovery as an indicator of a successful recovery process during yesterday's leisure time and aim at replicating past research by demonstrating positive relationships between morning recovery and work engagement (e.g., Garrick et al., 2014; Sonnentag, 2003; ten Brummelhuis \& Bakker, 2012b). Thus, we try to demonstrate that anticipated leisure time and yesterday's leisure time (i.e., morning recovery) account for unique parts in work engagement.

Finally, because existing research on upcoming leisure time and work engagement has been limited to one working day and one planned leisure activity per participant (Seibel et al., 2020), we followed two steps to extend existing research. First, we collect several leisure activities per participant using a daily diary approach over five days to check for within-person 
differences in pleasant anticipation. We further investigate ToPLA as a within-person mediator between pleasant anticipation and work engagement. Second, by adding general anticipation of leisure time, we refer to overall leisure time and consider that employees' leisure time contains more than just one leisure activity. Combining a diary study with two indicators of upcoming leisure time offers a comprehensive view of upcoming leisure time and help to understand the mechanism that may link upcoming leisure time and present work engagement. Our research model is summarised in Figure 1.

\section{Morning Recovery}

Recovery describes the process of unwinding from work-related stress when employees are free of work-related demands (Sonnentag \& Geurts, 2009). Apart from within-day work breaks (Trougakos \& Hideg, 2009), engaging in leisure activities provides opportunities to detach from work, relax, master new challenges, and experience control, helping employees prevent fatigue and make them more energetic (i.e., recovered) in the morning (for a meta-analysis, see Bennett et al., 2018). Thus, morning recovery indicates how successful the recovery process was during yesterday's leisure time and represents a resource (Sonnentag \& Fritz, 2015; Trougakos \& Hideg, 2009).

\section{Figure 1}

Research Model

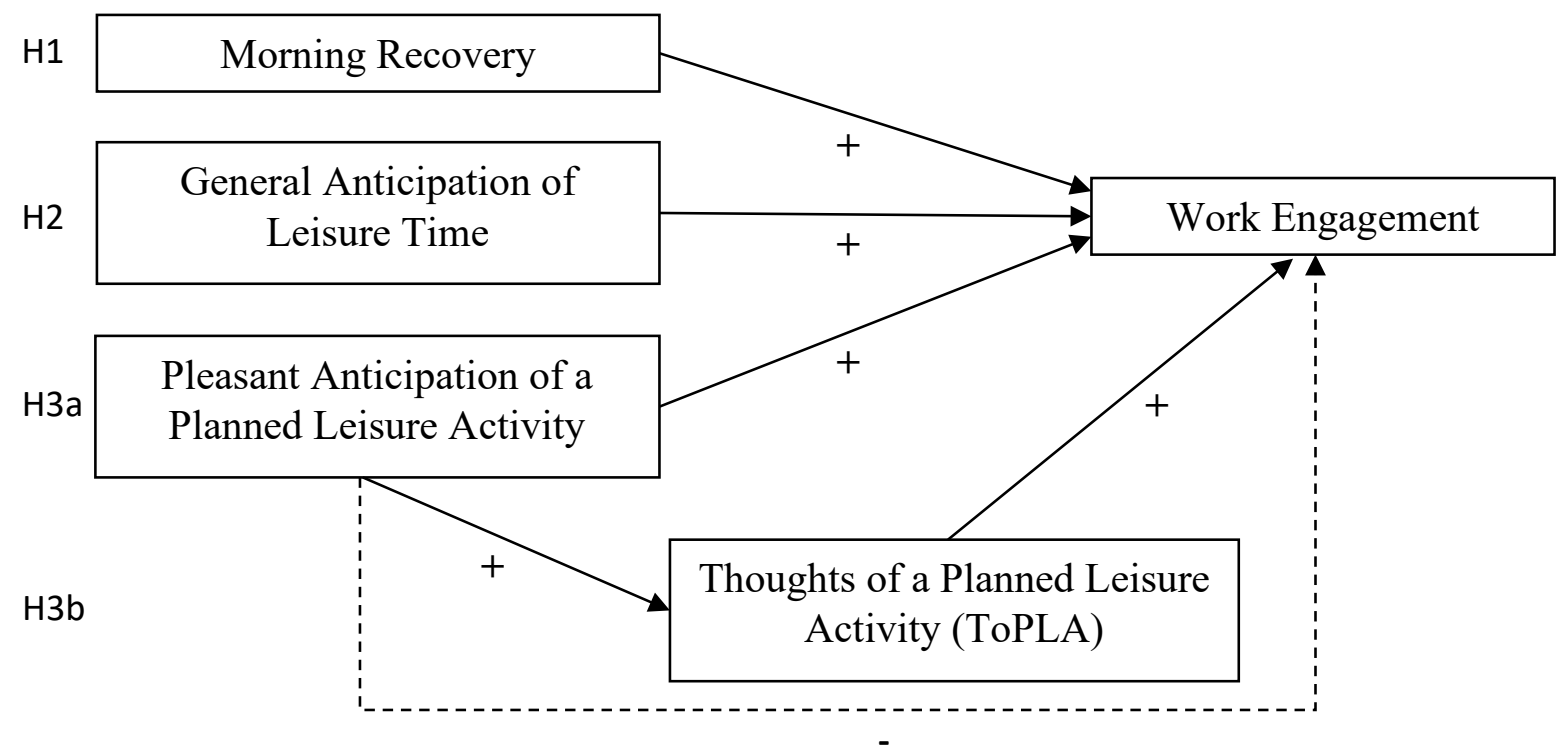

Note. The dotted line represents the indirect effect through ToPLA. 
During the working day, employees manage their resources by deciding whether to invest or save resources. Resources are defined broadly as everything that has a value for individuals (Hobfoll, 1989) and helps people reach their goals, e.g., social support, time away from work and recovery experiences (Halbesleben et al., 2014). According to COR theory (Hobfoll, 1989), employees stop investing resources (e.g., into their work) and enter a defensive mode of behaviour after their currently available resources fall below a specific resource limit. We define this turning point of resource investment as the lower resource limit. Because employees possess more resources in the morning after a successful recovery process (Breevaart et al., 2020; S. L. Parker et al., 2020), they can invest more resources into their work before they reach their lower resource limit, stop investing and start saving resources. Thus, in line with other research (e.g., Garrick et al., 2014; Sonnentag, 2003; ten Brummelhuis \& Bakker, 2012b), we propose that morning recovery is positively related to work engagement and formulate the following hypothesis:

Hypothesis 1: Day-level morning recovery will be positively related to day-level work engagement

\section{Anticipated Resource Gains}

According to COR theory (Hobfoll, 1989), not only present but also upcoming resources are relevant for resource management, and anticipated resource gains/losses may carry similar consequences as actual resource gains/losses (Gorgievski \& Hobfoll, 2008a; Hobfoll et al., 2003). Research has shown that, for instance, the threat of a resource loss (i.e., an anticipated resource loss) decreased performance in an experimental study (Niessen \& Jimmieson, 2016). Furthermore, the anticipation of less workload in the future (i.e., more time for potential resource gains) relates to lower strain in the present (DiStaso \& Shoss, 2020). Thus, evidence exists that anticipated resource gains/losses are associated with employees' current states and behaviours.

We concentrate on anticipated resource gains and assume that employees, who anticipate a resource gain, know that they will (re)gain resources soon and do not need to save (many) resources. In terms of COR theory (Hobfoll, 1989), employees' lower resource limit decreases in anticipation of a resource gain; therefore, they can invest more resources. While morning recovery directly increases employees' currently available resources, we posit that upcoming resource gains decrease employees' lower resource limit. Because leisure time and leisure activities provide opportunities to (re)gain resources, general anticipation of leisure time and pleasant anticipation of a planned leisure activity may indicate upcoming resource gains. 


\section{General Anticipation of Leisure Time}

Leisure time describes a time free from work, which employees could spend on their own choice (S. R. Parker \& Smith, 1976; Thierry \& Janson, 1998). Because employees do not need to invest further resources, they can replenish consumed resources (e.g., energy) and gain new resources (e.g., social support). Thus, employees may anticipate their entire leisure time as a source for potential resource gains. We define general anticipation of leisure time as employees' overall cognitive evaluation of their upcoming leisure time and propose that the general anticipation of leisure time indicates an anticipated resource gain. This cognitive evaluation of leisure time may depend on leisure time and current circumstances at work. For instance, when time pressure is high, employees may report greater anticipation of leisure time, as their upcoming leisure time indicates a reduction in time pressure. This example illustrates that the general anticipation of leisure time is not tied to a specific leisure activity but rather is an overall evaluation that depends on the present working day and future opportunities to (re)gain resources. In summary, when general anticipation of leisure time is high, employees expect high resource gains during leisure time. Consequently, employees' lower resource limit decreases, and they can invest more resources into their work engagement. Thus, we propose the following hypothesis:

Hypothesis 2: Day-level general anticipation of leisure time will be positively related to employees' day-level work engagement.

\section{Pleasant Anticipation of a Planned Leisure Activity}

Besides looking forward to their leisure time in general, employees may experience pleasant anticipation of one specific, planned leisure activity. Pleasant anticipation is defined as a positive affective reaction (e.g., joy, excitement), which is experienced only because of a positive, future event with a high probability of occurrence (Seibel et al., 2020). For instance, employees experience joy in the present (affective reaction) when they expect that meeting up with friends (future event) after work at 18:00 (high probability of occurrence) will be fun (positive evaluation of the event). Research on pleasant anticipation is rare but has demonstrated that pleasant anticipation relates to positive affect in the present, even though the anticipated event will happen in the future (Sonnentag, Mojza, et al., 2008; Syrek, Weigelt, et al., 2018).

We focus on pleasant anticipation of a planned leisure activity to cover anticipated resource gains from different planned leisure activities, as leisure activities provide different opportunities to (re)gain resources (for a review, see Demerouti et al., 2009). For instance, employees may regain energy resource during low-effort activities and social support during 
social activities. Pleasant anticipation combines different characteristics of the upcoming leisure activity (e.g., expectation of the outcome, probability of occurrence), and therefore, describes whether employees expect a high and probable resource gain from this leisure activity. We postulate that pleasant anticipation of a planned leisure activity indicates an upcoming resource gain, which reduces employees' lower resource limit and allows employees to invest more resources into their work engagement. Thus, we propose the following hypothesis:

Hypothesis 3a: Day-level pleasant anticipation of a planned leisure activity will be positively related to employees' work engagement.

\section{Thoughts of a Planned Leisure Activity (ToPLA) as a Mediator}

Research has demonstrated that employees with high pleasant anticipation of a planned leisure activity think more frequently about anticipated leisure activities (Seibel et al., 2020), which may distract them from the task at hand, reducing their work engagement. These thoughts are referred to as thoughts of a planned leisure activity (ToPLA) and are defined as "future-oriented thoughts of a specific plan that employees have in mind for their leisure time" (Seibel et al., 2020, p. 3). Pleasant anticipation is an antecedent of ToPLA for two reasons (Seibel et al., 2020): First, higher pleasant anticipation indicates a higher probability of occurrence for the planned leisure activity, i.e., employees have a more concrete cognitive representation of that leisure activity (see construal-level theory; Trope \& Liberman, 2010; Wiesenfeld et al., 2017). Because of the more concrete mental representation, employees recognize more environmental cues referring to their leisure activity, and therefore, ToPLA are more likely to be retrieved from memory (Chu et al., 2003; Howard \& Kahana, 1999). Second, as thoughts of positive (compared to negative) future events are generated more easily (Mitchell et al., 1997; NewbyClark \& Ross, 2003), high pleasant anticipation may also increase the frequency of ToPLA. Thus, we propose that pleasant anticipation is positively related to ToPLA.

During the working day, employees must remain focused on their tasks to demonstrate high performance (Beal et al., 2005), and off-task thoughts are negatively related to performance (for a meta-analysis, see Randall et al., 2014). ToPLA are a special type of offtask thoughts that drive attention away from the task at hand (Seibel et al., 2020). Therefore, when employees generate more ToPLA, they may concentrate less on their work and may show lower work engagement. In sum, we posit an alternative hypothesis. Specifically, we hypothesize that the relationship between pleasant anticipation of a planned leisure activity and work engagement is negative and mediated through employees' ToPLA: 
Hypothesis 3b: Day-level ToPLA will mediate the negative relationship between day-level pleasant anticipation of a planned leisure activity work engagement

\section{Method}

\section{Overview}

We conducted an online diary study comprising a pre-survey and three daily questionnaires across five consecutive workdays (Monday-Friday). After registration, participants were asked to choose a typical working week for participation (i.e., a week with regular working hours and without vacations) and were invited to fill out a pre-survey. During the five days of the study, participants received emails with links to three different questionnaires: a morning questionnaire (available from 6:00-10:00), a noon questionnaire (available from 11:00-14:00) and an afternoon questionnaire (available from 16:00-19:00). Participants were instructed to respond to the morning questionnaire at the beginning of their working day and to the afternoon questionnaire at the end of their working day. Data collection started in September 2019 and ended in May 2020, with a break in December and January due to public holidays. The registration was completed by 175 employees, and 138 employees filled out the pre-survey (78.86\%). Participants responded on average to 9.51 of 15 questionnaires (response rate: 63.40 ), and we collected data from 1369 single measurement points.

\section{Participants}

We recruited participants via flyers, social media, and personal contacts. Furthermore, four students helped us with data collection as part of their master's theses. Participants had to be over 18 and have regular working hours (i.e., 6:00-19:00) for at least 20 hours a week. Participation was voluntary, and participants could receive individual feedback on their work engagement and morning recovery and participate in a lottery $(4 \times 50 €)$. Demographic information was available for 134 employees. More than half the participants were female (59.71\%), and participants' average age was 37.84 years $(\mathrm{SD}=12.66$; range: $18-63)$. Most of them held a master's degree (48.51\%), a bachelor's degree (14.93\%), or finished a vocational training programme (16.42\%). Participants worked in a variety of occupational sectors, e.g., social and health care services $(20.90 \%)$, other service activities $(12.69 \%)$, and information and communication $(10.45 \%)$. On average, they worked 41.49 hours per week $(\mathrm{SD}=6.83)$. Some participants had leadership positions (28.36\%), and only a few were self-employed (7.46\%). 


\section{Measures}

In the pre-survey, we assessed the demographic variables. In the morning questionnaire, we asked about employees' morning recovery. In the noon questionnaire, we measured general anticipation of leisure time and pleasant anticipation of a planned leisure activity. In the afternoon questionnaire, employees' work engagement and their ToPLA were assessed. Unless otherwise stated, all questionnaires used a five-point Likert scale ranging from 1 (totally disagree) to 5 (totally agree).

\section{Morning Recovery}

Morning recovery was assessed with a scale developed by Sonnentag and Kruel (2006). The scale comprised four items, and one sample item read: "I feel mentally recovered". Withinperson reliability was .83 , and between-person reliability was $.78 .^{2}$

\section{General Anticipation of Leisure Time}

To measure employees' general anticipation of leisure time, we used a Kunin scale (Kunin, 1955), which consisted of seven different smiley faces from 1 (picture of a very unhappy smiley face) to 7 (picture of a very happy smiley face). The instruction read: "Overall, how much are you looking forward to your upcoming leisure time?". A similar measure was used previously to measure happiness during leisure activities (Oerlemans et al., 2014). Between-person reliability was .71, and because we used a single item measurement, within-person reliability could not be computed.

\section{Pleasant Anticipation of a Planned Leisure Activity}

We first asked participants to describe a planned leisure activity with three words. They mentioned, for example, "reading a book", "go shopping", or "do sports". Participants then were asked to classify their planned leisure activity into one of six categories (physical, social, low-effort, household, childcare, or work/administrative), a categorization schema commonly used in recovery research (e.g., Sonnentag, 2001; ten Brummelhuis \& Bakker, 2012b). Afterwards, we used a scale developed by Seibel et al. (2020) to measure pleasant anticipation of a planned leisure activity with four items. A sample item was "I am looking forward to my leisure activity". Within-person reliability was .84, and between-person reliability was 69 .

\footnotetext{
${ }^{2}$ We followed the recommendations by Nezlek (2017) for computing reliability in daily diary research. They recommend using formulas provided by Shrout and Lane (2012), which take into account not only different measurement points, but also the time nested within persons (i.e., the variance of the items and the time). Thus, within-person reliability indicates generalizability of within-person time variations averaged over items, and between-person reliability indicates generalizability of between-person differences averaged over time. We used the R package psych Revelle (2020) to conduct the reliability analyses.
} 


\section{Work engagement}

We used the short version of the Utrecht Work Engagement Scale (UWES-9; Schaufeli et al., 2006b) to measure work engagement. We selected employees' work engagement during the afternoon to refer to the period after the noon questionnaire. To address this period, we started each sentence with "In the afternoon" and removed three items, which did not fit into this period (e.g., "When I get up in the morning, I feel like going to work"). The scale comprised six items, e.g., "In the afternoon, I was immersed in my work". Within-person reliability was .85 , and between-person reliability was .84 .

\section{Thoughts of a Planned Leisure Activity (ToPLA)}

To measure employees' ToPLA, we used a single item (Seibel et al., 2020): "How frequently did you think of the planned leisure activity [here, the planned leisure activity from the noon questionnaire was displayed] that you indicated in the last questionnaire?". Participants could use an abstract rating scale from 1 (not at all) to 10 (all the time) to answer the question (i.e., participants could not indicate the concrete number of ToPLA). Between-person reliability was .71. Again, because we used a single item measurement, within-person reliability could not be computed.

\section{Results}

Our data was collected in a diary study (i.e., the same employee answers the same questionnaire several times); thus, the data comprised days (Level 1) nested within persons (Level 2), and we used a multilevel approach for our analyses (Raudenbush \& Bryk, 2002). Means, standard deviations, intraclass correlation coefficients (ICC) and correlations for all variables are presented in Table 1.

\section{Planned Leisure Activities}

We collected data from 456 different planned leisure activities. Participants classified 33.77\% as physical activities, $26.53 \%$ as social activities, $22.37 \%$ as low-effort activities, $11.62 \%$ as household activities, $3.95 \%$ as work or administrative activities, and $0.66 \%$ as childcare activities $(1.10 \%$ were not classified). Pleasant anticipation generally was high $(M=3.80, S D=0.69)$, and a univariate analysis of variance (ANOVA) revealed a significant difference in pleasant anticipation depending on the type of leisure activity, $F(1,5)=20.66, p$ $<.001$. Even though variances within the groups were equal, we did not conduct post-hoc contrast analyses because of the highly different group sizes. Descriptive analyses indicated that participants experienced the lowest pleasant anticipation for planned household activities $(M=3.05, S D=0.91)$ and planned administrative/work activities $(M=2.65, S D=1.09)$. 


\section{Table 1}

Means (M), Standard Deviations (SD), and Correlations of the Study Variables

\begin{tabular}{llcccccccc}
\hline \multicolumn{1}{c}{ Variable } & $M$ & $S D$ & ICC & 1 & 2 & 3 & 4 & 5 \\
\hline 1 & Recovery (T1) & 3.35 & 0.74 & .37 & & .06 & .04 & $.17^{* *}$ & -.02 \\
2 & General anticipation $^{\mathrm{b}}(\mathrm{T} 2)$ & 6.13 & 0.74 & .47 & $.23^{* *}$ & & $.54^{* *}$ & .06 & $.19^{* *}$ \\
3 & Pleasant anticipation $^{\mathrm{a}}(\mathrm{T} 2)$ & 3.81 & 0.68 & .29 & .14 & $.66^{* *}$ & & .06 & $.20^{* *}$ \\
4 & Work engagement (T3) $^{*}$ & 2.93 & 0.76 & .48 & $.26^{* *}$ & .12 & .08 & & -.10 \\
5 & ToPLA (T3) & 3.28 & 1.55 & .48 & -.10 & $.19^{*}$ & $.23^{*}$ & $-.25^{* *}$ & \\
\hline
\end{tabular}

Notes. T1 $=$ morning; T2 = noon; T3 = afternoon; ToPLA $=$ Thoughts of a planned leisure activity; ICC = intraclass correlation coefficient (percentage of variance between persons). Correlations below the diagonal are person-level (between-person) correlations $(N=113-133)$, and correlations above the diagonal are day-level (within-person) correlations $(N=307-447)$. a of a planned leisure activity.

of leisure time.

$* p<.05 . * * p<.01 . * * * p<.001$.

\section{Hypotheses testing}

We used multilevel path modelling in Mplus Version 7.4 (Muthén \& Muthén, 1998) to test all hypotheses in one model. A model with random intercept and fixed was used for our analyses. To test the mediation, we followed the recommendations by Preacher et al. (2010) and also modelled the mediation at Level 2 (2-2-2), although our main focus was on the lower-level mediation (1-1-1). The results for Level 1 are summarised in Figure 2, and the complete results from multilevel path modelling are presented in Table 2. Because the focus of our study was on daily changes within persons, we referred only to within-person effects when testing our hypotheses.

In Hypothesis 1, we assumed that daily morning recovery is positively related to daily work engagement. The path coefficient was significant within persons (estimate $=0.172$, $S E=0.068, p=.011$ ), supporting Hypothesis 1. In Hypothesis 2, we suggested that daily general anticipation of leisure time is positively related to daily work engagement. This relationship was significant within persons (estimate $=0.228, S E=0.104, p=.028$ ). Thus, Hypothesis 2 was supported.

For Hypotheses $3 \mathrm{a}$ and $3 \mathrm{~b}$, we proposed alternative hypotheses, positing that the relationship between daily pleasant anticipation of a planned leisure activity and work engagement is either positive (3a) or that the relationship is negative and mediated through employees' ToPLA (3b). The results showed a negative, non-significant relationship between pleasant anticipation and work engagement (estimate $=-0.094, S E=0.068, p=.171$ ), rejecting 
Figure 2

Results from Multilevel Path Analysis

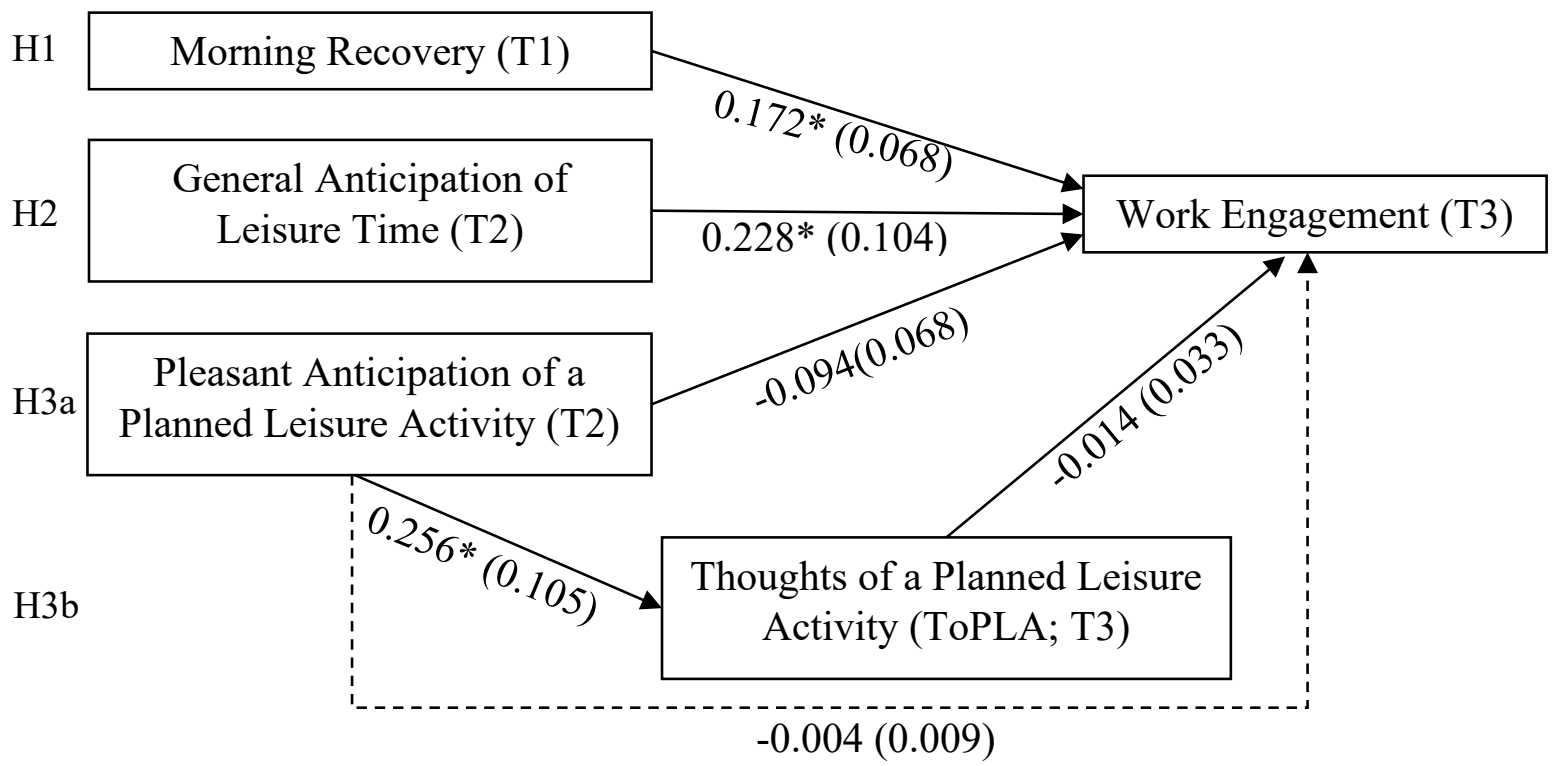

Note. $N=110$ employees; 302 matched questionnaires $(\mathrm{T} 1=$ morning, $\mathrm{T} 2=$ noon, and $\mathrm{T} 3$ = afternoon). Results show unstandardized estimates and standard errors in parentheses within persons. The dotted line represents the indirect effect through ToPLA. Model fit indices: $X^{2}=6.034, d f=4$, Akaike information criterion $=4045.692$, comparative fit index $=.949$, root mean square error of approximation $=.041$. $* p<.05$.

Hypothesis 3a. Regarding Hypothesis 3b, pleasant anticipation of a planned leisure activity was positively related to ToPLA within persons (estimate $=0.256, S E=0.105, p=.015$ ). However, ToPLA was not related to work engagement within persons (estimate $=-0.014, S E=0.033$, $p=.675)$, and the indirect effect between pleasant anticipation and work engagement through ToPLA was not significant (indirect effect $=-0.004, S E=0.009, p=.681$ ). Hence, Hypothesis $3 \mathrm{~b}$ was rejected. At the between-person level, only the relationship between recovery and work engagement was significant (estimate $=0.407, S E=0.169, p=.016$ ). 
Table 2

Results from Multilevel Path Analyses

\begin{tabular}{|c|c|c|c|c|}
\hline Effect & Estimate & $S E$ & $p$ & $95 \% \mathrm{CI}$ \\
\hline \multicolumn{5}{|l|}{ Within-person effects } \\
\hline Recovery $\rightarrow$ Work engagement & 0.172 & 0.068 & .011 & {$[0.060 ; 0.284]$} \\
\hline General anticipation $^{\mathrm{b}} \rightarrow$ Work engagement & 0.228 & 0.104 & .028 & {$[0.057 ; 0.399]$} \\
\hline Pleasant anticipation $^{\mathrm{a}} \rightarrow$ Work engagement & -0.094 & 0.068 & .171 & {$[-0.206 ; 0.019]$} \\
\hline Pleasant anticipation $^{\mathrm{a}} \rightarrow$ ToPLA & 0.256 & 0.105 & .015 & {$[0.082 ; 0.429]$} \\
\hline ToPLA $\rightarrow$ Work engagement & -0.014 & 0.033 & 675 & {$[-0.068 ; 0.040]$} \\
\hline $\begin{array}{l}\text { Pleasant anticipation }{ }^{\mathrm{a}} \rightarrow \text { ToPLA } \\
\rightarrow \text { Work Engagement }\end{array}$ & -0.004 & 0.009 & .681 & {$[-0.018 ; 0.011]$} \\
\hline \multicolumn{5}{|l|}{ Between-person effects } \\
\hline Recovery $\rightarrow$ Work engagement & 0.407 & 0.169 & .016 & {$[0.129 ; 0.686]$} \\
\hline General anticipation $^{\mathrm{b}} \rightarrow$ Work engagement & 0.030 & 0.249 & .904 & {$[-0.379 ; 0.439]$} \\
\hline Pleasant anticipation $^{\mathrm{a}} \rightarrow$ Work engagement & 0.143 & 0.364 & 694 & {$[-0.456 ; 0.742]$} \\
\hline Pleasant anticipation $^{\mathrm{a}} \rightarrow$ ToPLA & 0.913 & 0.393 & .020 & {$[0.266 ; 1.559]$} \\
\hline ToPLA $\rightarrow$ Work engagement & -0.151 & 0.088 & .088 & {$[-0.296 ;-0.006]$} \\
\hline $\begin{array}{l}\text { Pleasant anticipation }{ }^{\mathrm{a}} \rightarrow \text { ToPLA } \\
\rightarrow \text { Work engagement }\end{array}$ & -0.138 & 0.100 & .166 & {$[-0.302 ; 0.026]$} \\
\hline
\end{tabular}

Note. $N=110$ employees; 302 matched questionnaires (morning, noon, and afternoon).

Results show unstandardized estimates. Model fit indices: $X^{2}=6.034, d f=4$, Akaike information criterion $=4045.692$, comparative fit index $=.949$, root mean square error of approximation $=.041$.

${ }^{\mathrm{a}}$ of a planned leisure activity.

${ }^{b}$ of leisure time.

\section{Discussion}

The overarching goal of our research was to answer the question of whether both, upcoming leisure time and yesterday's leisure time, relate to work engagement. First, morning recovery (a consequence of yesterday's leisure time) and work engagement were positively associated, concurring with a large amount of previous research (e.g., Garrick et al., 2014; Sonnentag, 2003; ten Brummelhuis \& Bakker, 2012b). When employees started recovered in the working day, more resources were available (e.g., more energy), which boosted their work engagement. Second, daily general anticipation of leisure time related positively to work engagement, and employees who expected a positive, upcoming leisure time were more engaged in their work. In terms of COR theory (Hobfoll, 1989), employees' lower resource limit, which indicates the turning point of resource investment, decreased because of anticipated resource gains. Therefore, employees could invest more resources without the threat of investing more resources than they could (re)gain during their leisure time. Finally, we assumed that daily 
pleasant anticipation of a planned leisure activity is related either positively or negatively to work engagement and that the negative relationship is mediated through ToPLA. In contrast to our expectations, the results from multilevel path modelling supported none of these hypotheses, and daily pleasant anticipation was not related to work engagement. When employees looked forward to a specific, planned leisure activity, their work engagement did not change.

There are several explanations why pleasant anticipation was not related to work engagement. First, pleasant anticipation refers to only one planned leisure activity, and therefore, pleasant anticipation covers only one part of employees' leisure time. While participants might report pleasant anticipation for a positive leisure activity, negative activities during leisure time might also exist, so overall work engagement did not increase. Because most employees mentioned a positive planned leisure activity when asked for pleasant anticipation, negative (i.e., resource-draining) leisure activities might have been neglected. Second, employees indicated many different leisure activities across the week, which address different resources (e.g., energy, social support), and we referred to pleasant anticipation to measure the anticipated resource gains from these different leisure activities. Because only some anticipated resource gains may relate to work engagement, the relationship between pleasant anticipation and work engagement might be non-significant. Third, one may also speculate that the resource management process depends on employees' implicit theories about their resources (see implicit theories about willpower; Job et al., 2010). While some employees may assume that leisure activities could replenish invested resources and benefit from pleasant anticipation, other employees may believe that resource could not be re(gain)ed. Thus, differences between persons may explain the non-significant relationship.

The non-significant relationship between pleasant anticipation of a planned leisure activity and work engagement points out the difference between pleasant anticipation and general anticipation of leisure time. In contrast to pleasant anticipation, general anticipation refers to participants entire leisure time, including all positive and negative activities and events in the upcoming leisure time. Therefore, the cognitive evaluation of these activities and events is an overall rating of the anticipated resource gains employees expect. We suppose that this overall rating has much more impact than the evaluation of single leisure activities on employees' resource management.

Apart from the positive relationship between pleasant anticipation of a planned leisure activity and work engagement, we also argued that this relationship might be negative and mediated by employees' ToPLA. Pleasant anticipation was positively related to ToPLA; thus, 
looking forward to a positive leisure activity triggered thoughts about that activity. However, ToPLA were not related to employees' work engagement, and ToPLA did not meditate the relationship between pleasant anticipation and work engagem 3 ent.

The positive relationship between pleasant anticipation and ToPLA corresponds to a previous study (Seibel et al., 2020); however, in contrast to our results, that study also revealed a negative and significant relationship between hourly ToPLA and hourly work engagement. The focus of our study on differences between days (not hours) may explain the non-significant relationship between ToPLA and work engagement. First, because we asked employees for the frequency of ToPLA in the afternoon, it could be difficult for them to estimate the frequency of ToPLA for the full working day (compared to an hourly measurement of ToPLA). As a result, they might have underestimated the frequency of ToPLA. Second, we assessed ToPLA once a day, and each measurement of ToPLA referred to a different planned leisure activity. One may speculate that the content of ToPLA is more critical for work engagement than the frequency. For instance, the frequency of ToPLA could be the same on two days, but ToPLA refers to positive imaginations of the planned leisure activity on one day and doubts about the occurrence of the planned activity on the other. While the positive imaginations may enhance work engagement, the doubts may distract employees from their work. In summary, we did not find a direct nor indirect relationship between pleasant anticipation and work engagement. Nevertheless, our study demonstrated that high pleasant anticipation of a planned leisure activity was related to more ToPLA, a type of off-task thoughts.

\section{Theoretical Implications}

Our study provided evidence that not only past but also anticipated resource gains are meaningful, which concurs with COR theory (Gorgievski \& Hobfoll, 2008a; Hobfoll et al., 2003). We demonstrated that employees take their anticipated leisure time into account while they are still at work and that employees' daily general anticipation of leisure time is beneficial for daily work engagement. More specifically, employees' daily work engagement depends on two different mechanisms: employees' currently available resources (i.e., morning recovery), which they can directly invest into work engagement, and employees' anticipated resource gains, which reduce their lower resource limit without providing additional resources. The two mechanisms carry different implications for resource management, and we propose to pay attention to this differentiation when using COR theory (Hobfoll, 1989) to explain behaviour.

We also showed that morning recovery could be considered a resource in COR theory (Hobfoll, 1989) as proposed by other researchers (Sonnentag \& Fritz, 2015; Trougakos \& 
Hideg, 2009). This finding highlights the importance of leisure time in general, as leisure time provides many opportunities to recover and (re)gain resources (e.g., Demerouti et al., 2009; Steed et al., 2019). Furthermore, the relationship between recovery and work engagement was significant at a between-person level, indicating that employees with a higher morning recovery over the working week were more engaged than employees with lower morning recovery. Thus, average and day-level morning recovery are crucial für employees' morning recovery.

\section{Practical Implications}

Our study showed that the general anticipation of leisure time and morning recovery were associated with higher work engagement and revealed that pleasant anticipation of a planned leisure activity related to ToPLA. Therefore, the practical implications are twofold: On the one hand, leisure time should include activities that foster recovery and resource replenishment to start the next working day with a high level of morning recovery (for a review, see Demerouti et al., 2009). These activities should be embedded in an overall positive leisure time such that employees could look forward to this upcoming leisure time and anticipate a resource gain. Even though leisure time may include negative leisure activities (e.g., cleaning chores, doing laundry), employees could enrich their leisure time with smaller positive components (e.g., calling a friend, listening to music).

On the other hand, specific planned leisure activity combined with high pleasant anticipation should be considered critical. ToPLA did not relate to work engagement, yet ToPLA are a type of off-task thoughts that can drive the attention away from the current task (Randall et al., 2014). Thus, employees should try to reduce the frequency of ToPLA to stay concentrated at work, although ToPLA may not directly affect their work engagement. Therefore, we propose that employees plan their leisure activities in the morning, using a fixed time (e.g., five minutes in the morning) to concentrate on the planned leisure activity. Furthermore, to prevent a conflict between leisure and work-related plans, it may be beneficial when employees postpone their extraordinary leisure plans, which are more likely to evoke high pleasant anticipation, to the weekend.

\section{Limitations and Future Research}

We investigated general anticipation of leisure time as well as pleasant anticipation of a planned leisure activity. From a theoretical perspective, planned leisure activities are part of employees' leisure time, and on days with high pleasant anticipation, general anticipation should also be high (explaining the within-person correlation). Furthermore, individuals differ in their ability to enjoy or savour future events (Bryant, 2003), and individuals who can better enjoy their 
anticipated leisure time may also enjoy their planned leisure activity more (explaining the between-person correlation). From an empirical perspective, only the general anticipation of leisure time related positively to work engagement, demonstrating that the differentiation between pleasant anticipation and general anticipation is necessary. In summary, we assume that both constructs are different, and we suggest that future research should consider them distinct.

To keep our daily questionnaires short and to enhance participants' compliance (Ohly et al., 2010), we used two single-item measurements throughout our study (Kunin scale for general anticipation, abstract rating scale for ToPLA). Between-person reliabilities for these items were acceptable, but we could not compute within-person reliabilities. However, within- and between-person reliabilities typically are not completely different (e.g., Liu et al., 2020; Ouyang et al., 2019), suggesting that the two single-item measurements may also be generalizable within persons. Furthermore, Kunin scales are commonly used to assess general evaluations, e.g., job satisfaction (for a meta-analysis, see Wanous et al., 1997) or overall leader satisfaction (van Quaquebeke et al., 2010). Thus, we assume that the Kunin scale was appropriate to measure the general anticipation of leisure time. For ToPLA, future research could develop additional items based on questionnaires that measure work-related thoughts during leisure time (e.g., Fritz \& Sonnentag, 2006; Querstret \& Cropley, 2012).

Finally, all of our variables of interest referred to employees' affective reactions (e.g., pleasant anticipation), states (work engagement), and thoughts (ToPLA) and were best accessible through self-ratings. To reduce common method variance (Podsakoff et al., 2012), we measured our predictor variables and our criterium variable at different measurement points and used different scale formats (Likert-scale, Kunin scale, abstract rating scale). Future research could expand our research on work engagement to outcomes rated by co-workers and supervisors.

\section{Conclusion}

The present diary study demonstrated that morning recovery and general anticipation of leisure time are positively associated with work engagement. Thus, leisure time has two functions for employees at two different times. First, during the working day, leisure time indicates an upcoming resource gain. Second, after the working day, leisure time offers opportunities to realize this resource gain. 


\section{Acknowledgement}

We thank Jetmir Zyberaj for his helpful comments on an earlier version of this article. Furthermore, we thank Magdalena Gilch, Mira Stümpfig, and Catharina Vohs for their help with data collection.

\section{Author Contributions}

Sebastian Seibel developed the theoretical background and hypothesis for this study; Judith Volmer and Sebastian Seibel planned the Study; Sebastian Seibel conducted the study and analysed the data; Sebastian Seibel wrote a first draft of the manuscript; Judith Volmer revised earlier versions of the submitted manuscript; both authors have read the final version and agreed to the published version of the manuscript.

\section{Funding}

This research received no external funding.

\section{Institutional Review Board Statement}

The study was conducted according to the guidelines of the Declaration of Helsinki, and approved by the Ethics Committee of Otto-Friedrich University of Bamberg (Dossier number 2021-05/21; date of approval: 30 May 2021).

\section{Informed Consent Statement}

Informed consent was obtained from all subjects involved in the study.

\section{Conflicts of Interest}

The authors declare no conflict of interest. 


\section{6}

\section{GENERAL DISCUSSION}

Previous recovery research has demonstrated that past leisure time and leisure activities are crucial for employees' recovery, psychological well-being, and general health (for a metaanalysis, see Steed et al., 2019). Because upcoming events can also change employees' feelings, thoughts, and behaviour (e.g., Baumeister et al., 2007; Casper \& Sonnentag, 2020; DiStaso \& Shoss, 2020; Oettingen, 2012), employees may anticipate leisure time and leisure activities. Building on the conservation of resources (COR) theory (Hobfoll, 1989; Hobfoll et al., 2018), I hypothesized that upcoming leisure time and leisure activities indicate upcoming resource gains and enhance employees' work engagement, a positive, motivational work-related state (Schaufeli et al., 2006b) associated with employees' health (for a meta-analysis, see Halbesleben, 2010b) and performance (for a meta-analysis, see Christian et al., 2011). In sum, the overarching goal of this dissertation was to study whether the anticipation of leisure time is associated with present work engagement. Therefore, pleasant anticipation of a planned leisure activity (Study 1, 2, 3), leisure thoughts (Study 2, 3), and general anticipation of leisure time (Study 3) were investigated.

\section{Summary of the Results}

In Study 1, the relationship between pleasant anticipation of a planned leisure activity and work engagement was examined. Pleasant anticipation is a positive, affective reaction because of an upcoming event (Baumgartner et al., 2008) and was supposed to indicate an upcoming resource gain. Furthermore, employees' recovery-related self-efficacy (RRSE; Sonnentag \& Kruel, 2006), individuals' beliefs about their ability to recover during leisure time, was suggested to moderate this relationship.

Pleasant anticipation was positively related to work engagement between persons on one day and across the week. Thus, an employee with higher pleasant anticipation on one day or across the week reported higher work engagement than an employee with lower pleasant anticipation on one day or across the week. In contrast, pleasant anticipation was not associated with work engagement within persons, indicating that the same employee did not show higher work engagement on days with higher pleasant anticipation than on days with lower pleasant anticipation. In addition, employees' RRSE did not moderate this relationship at any level of 
analysis. Thus, employees' beliefs in their ability to recover from work stress did not interact with the expectations of their future leisure time. However, RRSE was positively related to work engagement at the between-person level, which showed that employees differed in their RRSE and that these differences explained work engagement differences.

In Study 2, the concept of leisure thoughts was introduced, and antecedents (time of the day, pleasant anticipation) and consequences of leisure thoughts (work engagement) were investigated. Hence, two different types of leisure thoughts were defined: thoughts of leisure time (ToLT), which refer to all thoughts about the upcoming leisure time, and thoughts about a planned leisure activity (ToPLA), which refer only to thoughts about one specific, planned leisure activity.

First, the results revealed that employees think more frequently of their leisure time (ToLT) and leisure activities (ToPLA) at the beginning and the end of the working day when work and leisure are closer in time. Second, pleasant anticipation was positively related to ToPLA, yet not to ToLT, demonstrating that ToLT and ToPLA are different types of leisure thoughts. Thus, employees who feel elated, happy, and/or excited because of an upcoming leisure activity think more frequently about that activity but not more frequently about their leisure time in general. Third, when employees thought more frequently about their leisure time (frequent ToLT), they were less engaged in their work across the working day. In addition, when employees thought more about their planned leisure activity within one hour (frequent ToPLA), they were less engaged in the same hour. Finally, because ToPLA may remind employees of an upcoming resource gain, it was hypothesized that ToPLA have positive consequences for employees' work engagement in the subsequent hour for leisure activity with a positive leisure plan valence. However, the results revealed that employees were even more distracted from their work (i.e., reported lower work engagement) when they thought of positive leisure activities.

The aim of Study 3 was to further investigate ToPLA and examine ToPLA as a mediator in the relationship between pleasant anticipation and work engagement. In addition, general anticipation of leisure time was introduced as an overall cognitive evaluation of employees' upcoming leisure time. While pleasant anticipation refers to one planned leisure activity, general anticipation covers the entire leisure time. Furthermore, to contrast past and upcoming leisure time, morning recovery was assessed to study whether past leisure activities (as indicated by morning recovery) and upcoming leisure activities (as indicated by pleasant anticipation, general anticipation) are simultaneously associated with work engagement. 
In sum, Study 3 revealed that pleasant anticipation was positively related to ToPLA within persons. However, ToPLA was not associated with work engagement and did not mediate the relationship between pleasant anticipation and work engagement. Thus, employees who experienced more pleasant anticipation thought more frequently of their planned leisure activity but did not report lower work engagement. Furthermore, general anticipation of leisure time was positively related to work engagement within persons, indicating that employees, who expect a positive leisure time, were more engaged in their work. Moreover, morning recovery was positively related to work engagement within and between persons. Employees reported higher work engagement on days with high recovery in the morning than on days with low recovery. In addition, employees with high recovery across the week were more engaged in their work than employees with low recovery. Interestingly, morning recovery and general anticipation of leisure time were simultaneously related to work engagement within persons, indicating that past and upcoming leisure time is essential for employees' work engagement. In summary, the findings from all three studies demonstrated that the anticipation of leisure time may relate to present work and may have positive (in the case of pleasant anticipation betweenpersons, general anticipation within-persons) or negative (in the case of ToLT across the day, ToPLA within one hour) consequences for work engagement.

\section{Theoretical implications}

Each of the studies included in this dissertation has unique theoretical implications, but the focal theoretical implications relate to the three overarching research goals. The first and central goal referred to the association between anticipated leisure time (i.e., pleasant anticipation and general anticipation) and work engagement. The results demonstrated that pleasant anticipation (between-persons) and general anticipation (within-persons) were associated with work engagement, indicating that upcoming leisure time matters for present work engagement. Because research has only investigated the relationship between past leisure activities and work engagement until now (e.g., Oerlemans et al., 2014; Sonnentag, 2003; ten Brummelhuis \& Bakker, 2012b), these findings add to the literature on work engagement and leisure activities (for a review, see Demerouti et al., 2009) by shedding light on anticipated leisure activities and leisure time.

In the past years, research in work and organisational psychology has shown a growing interest in upcoming work-related events or conditions, for example, the anticipation of workload (Casper \& Sonnentag, 2020; DiStaso \& Shoss, 2020), time pressure (Leroy \& Glomb, 2018b), and stress (Chen et al., 2009). Regarding the anticipation of leisure-related events, 
research has only investigated rare and special events, such as vacations (Besser et al., 2016; Nawijn et al., 2013) and Christmas (Bryant, 2003; Syrek, Weigelt, et al., 2018) but neglected leisure time as an ordinary, recursive period of the day. The present dissertation expanded this research on the anticipation of upcoming events by highlighting the role of daily upcoming leisure time. Furthermore, the findings that upcoming leisure time may affect employees while they are at work agree with a large amount of research in psychology, demonstrating that upcoming events and thoughts about the future change present feelings, thoughts, and behaviour (e.g., Bagozzi et al., 1998; Baumeister et al., 2007; Kong et al., 2011a; Oettingen, 2012; van Boven \& Ashworth, 2007).

To investigate the anticipation of upcoming leisure activities, pleasant anticipation of a planned leisure activity was introduced in Study 1 . Research on the anticipation of future events has focused on the outcomes of the future event (Besser et al., 2016; Koenig-Lewis \& Palmer, 2014; Monfort et al., 2015; Sonnentag, Mojza, et al., 2008), anticipatory and anticipated affective reactions (e.g., Baumgartner et al., 2008; Carrera et al., 2012; Xu \& Guo, 2019), or the probability of occurrence for the upcoming event (Maglio \& Polman, 2016; Muris \& van der Heiden, 2006). Because pleasant anticipation combines affective reactions with characteristics of the future event (positive outcome, high probability of occurrence), it integrates different characteristics of the anticipated event into one construct. Based on a short reference to pleasant anticipation (Baumgartner et al., 2008), an extended definition of pleasant anticipation was developed in Study 1, differentiating pleasant anticipation from other affective reactions. Because pleasant anticipation is not limited to leisure activities, it could be applied to investigate other upcoming events at work and leisure time. Thus, pleasant anticipation could be complemented to the list of positive future-oriented emotions (Graham et al., 2019).

The second overarching research goal referred to whether employees think of their upcoming leisure time during work. Hence, I introduced the concept of leisure thoughts in Study 2 and differentiated between ToLT and ToPLA. Leisure thoughts (ToLT, ToPLA) were defined as a specific type of off-task thoughts, which concurs with existing research on on- and off-task thoughts (e.g., Beal et al., 2005; Gardner et al., 1989; Kanfer \& Ackerman, 1989). The findings revealed that different types of thoughts (ToLT, ToPLA, thoughts on work, thoughts on other things) varied in their association with work engagement. Thus, instead of investigating the differences between on- and off-task thoughts, it is also important to consider fine graduations in various thoughts employees experience at work. Furthermore, the negative relationship between off-task thoughts and work engagement is in line with research showing that off-task thoughts are harmful to performance (for a meta-analysis, see Randall et al., 2014). Regarding 
the assumption that some off-task thoughts may have positive consequences for employees' performance (Dane, 2018), the findings from Study 2 excluded leisure thoughts as off-task thoughts with positive consequences for employees' work engagement and, because work engagement and performance are associated (Bailey et al., 2017; Christian et al., 2011), partly for employees' performance.

The idea of leisure thoughts generally expanded research on spontaneous thoughts (e.g., D’Argembeau, 2018; Dane, 2018; Marchetti et al., 2016; Mrazek et al., 2012) by emphasizing that different types of leisure thoughts, namely ToLT and ToPLA, exist. Furthermore, ToLT and ToPLA followed a time trend, and leisure thoughts were experienced more frequently at the beginning and the end of the working day. While the time of the day has been neglected as an explanation for the frequency of spontaneous thoughts in previous research (Barsics et al., 2016; D'Argembeau et al., 2011), the relationship between time of day and performance has been discussed (for a review, see A. P. Smith, 2013). Regarding work engagement, initial evidence exists that work engagement is lower in the morning and the evening than at noon (Syrek, Kühnel, et al., 2018), which concurs with Study 2. Thus, Study 2 showed that time could be an antecedent of off-task thoughts and introduced leisure thoughts as one explanatory mechanism for the time trend in work engagement. In sum, leisure thoughts are an important extension to research on off-task thoughts, and more research on different types of thoughts in the context of work and organisational psychology is needed in the future.

The third research goal was to examine ToPLA as a mediator in the relationship between pleasant anticipation and work engagement and to study whether past and upcoming leisure activities explain different parts of work engagement. Study 3 revealed that morning recovery (i.e., a consequence of past leisure activities) and general anticipation of leisure time were simultaneously associated with work engagement within persons. Thus, general anticipation explained additional variance in work engagement beyond morning recovery. These findings yield support for the focal idea of this dissertation that upcoming leisure time is crucial for work engagement. Furthermore, the results may hint at why the relationship between pleasant anticipation of a planned leisure activity and work engagement was non-significant within persons. When employees indicated their pleasant anticipation (i.e., the anticipated resource gain), they referred to one specific, planned leisure activity (e.g., cooking, reading a book). However, this activity reflected only one part of employees' entire leisure time, and other activities may indicate additional anticipated resource gains and losses. Thus, when indicating pleasant anticipation, further resource gains and losses were not considered. Because changes 
in employees' work engagement may depend on all anticipated resource gains and losses, general anticipation of leisure time may be a better indicator for work engagement changes.

Furthermore, ToPLA did not mediate the within-person relationship between pleasant anticipation and work engagement, although pleasant anticipation was positively related to ToPLA, which replicated Study 2. Thus, pleasant anticipation is a stable antecedent of ToPLA. However, in contrast to Study 2, ToPLA were not significantly related to work engagement in Study 3, which the different levels of analysis may explain: The relationship between ToPLA and work engagement was examined on an hourly level in Study 2 and across the day in Study 3. Remembering how frequently ToPLA occurred during the entire day might be more difficult for employees than remembering the frequency of ToPLA in the last hour. Therefore, they might underestimate the frequency of ToPLA, explaining the non-significant relationship between ToPLA and work engagement in Study 3.

Moreover, Study 1 and Study 3 examined pleasant anticipation, and the results revealed differences and similarities. First, at the between-person level, pleasant anticipation was positively related to work engagement in Study 1, yet not in Study 3. One explanation may be that the general anticipation of leisure time was simultaneously added to a multilevel structural equation model in Study 3. Because planned leisure activities are part of the entire leisure time, general anticipation incorporates pleasant anticipation, and both constructs partly share the same variance in work engagement. This overlap between the constructs may be crucial at the between-person level of analysis, reflecting differences between employees in average pleasant anticipation and average general anticipation across the week. One may argue that while daily differences between pleasant anticipation and general anticipation are meaningful, these differences are balanced out across the week. As a result, neither pleasant anticipation nor general anticipation was associated with work engagement between persons. Regarding the within-person relationship, Study 1 concurs with Study 3, and both studies revealed a nonsignificant relationship within persons. Thus, pleasant anticipation of planned leisure activities seems not to explain day-to-day variations in employees' work engagement.

Apart from answering the three overarching research questions, this dissertation has general implications for the conservation of resources theory (COR theory; Hobfoll, 1989; Hobfoll et al., 2018) and for the relationship between employees' resources and work engagement (for a review, see Bailey et al., 2017). The theoretical framework for all three studies was the COR theory (Hobfoll, 1989; Hobfoll et al., 2018), and pleasant anticipation of a planned leisure activity and general anticipation of leisure time were supposed to indicate an upcoming resource gain. Regarding COR theory (Hobfoll, 1989; Hobfoll et al., 2018) and 
pleasant anticipation, two main conclusions could be drawn. First, average pleasant anticipation across the working week may reflect a resource, indicating that employees have an overall positive view of their anticipated leisure time across a week. Second, because daily pleasant anticipation was not related to work engagement, it remains unclear whether pleasant anticipation indicates an upcoming resource gain. Furthermore, the general anticipation of leisure time could be regarded as an anticipated resource gain within persons. In addition, Study 1 revealed that RRSE could be added to the long list of resources (Halbesleben et al., 2014), which employees could use, for instance, to enhance their work engagement. In sum, the findings demonstrated that anticipated resource gains may be important in terms of COR theory (Hobfoll, 1989; Hobfoll et al., 2018) and that anticipated leisure time may indicate upcoming resource gains.

Employees' work engagement was investigated in all three studies, and research has shown that employees' resources are essential for high work engagement (for meta-analyses, see Crawford et al., 2010; Halbesleben, 2010a; Lesener et al., 2019). In line with this, present resources (morning recovery, RRSE) were positively related to work engagement. Because work engagement also is suggested to result from an anticipated resource gain (Hu et al., 2017), the anticipation of leisure time was investigated. The findings provide evidence that general anticipation of leisure time, assumed to indicate an upcoming resource gain, was positively related to work engagement within persons. However, the mechanism which connects general anticipation of leisure time (i.e., an anticipated resource gain) with work engagement was not examined in this dissertation and remains unclear. The anticipated resource gain may affect employees' thoughts and feelings, which may explain the positive relationship between general anticipation of leisure time and work engagement. One may speculate that general anticipation of leisure time activates an overall positive mindset for the working day or stimulates positive affect.

In summary, although the importance of future events has often been discussed (e.g., Bagozzi et al., 1998; Baumeister et al., 2007; Oettingen et al., 2001), recovery research has only investigated the relationship between past events, such as leisure activities and micro-breaks, and work engagement (e.g., Oerlemans et al., 2014; Sonnentag, 2003; ten Brummelhuis \& Bakker, 2012b). Because all three studies emphasized the importance of future events (i.e., leisure time and planned leisure activities) and future-oriented leisure thoughts (ToLT, ToPLA), either increasing or diminishing work engagement, this dissertation showed that it could be beneficial to broaden the scope to upcoming leisure time. Until now, research has demonstrated 
that present resources are essential to employees' work engagement (e.g., Crawford et al., 2010; Lesener et al., 2019), yet this dissertation extended this view to anticipated resource gains.

\section{Practical Implications}

The findings of this dissertation have several practical implications, which can be integrated into employee recovery training (e.g., Ebert et al., 2015; Hahn et al., 2011; Siu et al., 2014) and into work engagement interventions (for a review, see Knight et al., 2019). Commonly, recovery training focuses on detachment from work (i.e., recovery experiences; Hahn et al., 2011; Siu et al., 2014) and the importance of planning and integrating recovery in everyday life (e.g., leisure activities; Ebert et al., 2015; Thiart et al., 2013). However, thinking of these planned leisure activities may also decrease employees' work engagement. Consequently, employees may not finish their tasks, triggering ruminative thoughts during leisure time that interfere with recovery (Peifer et al., 2019; Weigelt et al., 2019a). Thus, the focus of planning leisure activities in recovery training could be problematic. Therefore, I suggest expanding the psychoeducation in recovery training (Ebert et al., 2015; Hahn et al., 2011; Thiart et al., 2013) with explanations about leisure thoughts and their relationship with work engagement.

Apart from the negative consequences of leisure thoughts, the findings also revealed that planned leisure activities (between-persons) and general anticipation (within-persons) are beneficial for work engagement. Hence, there are two other interesting practical implications. First, and in line with the existing recovery training (Ebert et al., 2015; e.g., Hahn et al., 2011; Siu et al., 2014), employees should learn how to integrate leisure activities into their everyday life. As a result, these leisure activities may become a daily routine, and employees may engage more frequently in leisure activities, which is beneficial for two reasons. On the one hand, leisure activities are positively related to morning recovery (e.g., Sonnentag, 2001; Sonnentag, Binnewies, \& Mojza, 2008), and Study 3 showed that morning recovery is important for work engagement. On the other hand, overall pleasant anticipation of planned leisure activities across a week was positively associated with work engagement. Thus, when leisure activities are part of everyday life, employees have more opportunities to experience pleasant anticipation. Furthermore, routinized leisure activities also help employees foster their RRSE (Hahn et al., 2011), and RRSE was positively related to work engagement in Study 1.

Second, employees may also learn how to benefit from anticipated leisure time. Enjoying future events before they occur is known as savouring (Bryant \& Veroff, 2006a), and several interventions exist that improve savouring (for a review and meta-analysis, see J. L. Smith \& Hanni, 2019). Thus, recovery training should also teach employees how to savour their 
upcoming leisure time. For instance, based on best-positive-self interventions (King, 2001; Sheldon \& Lyubomirsky, 2006), employees could write about their best positive and possible leisure time. They could imagine the best outcome of their leisure activities and how they may feel before, during, and after engaging in their leisure activities. As a result, employees' ability to experience general anticipation may be improved, and, in turn, their work engagement may be fostered.

Finally, to deal with leisure thoughts, one may speculate that allocating a fixed time to plan and think about leisure time may help employees experience fewer leisure thoughts during the working day. For instance, when employees plan their leisure time in the morning, making leisure plans is no longer an unfinished task, which may trigger ruminative thoughts (Weigelt et al., 2019a). Thus, information about how and when to plan leisure activities should be part of recovery training. Furthermore, recovery as a routine in everyday life may have an interesting side effect on pleasant anticipation of a planned leisure activity. Because employees do not have to plan routinized leisure activities, they may also think less frequently about the upcoming leisure time. Moreover, it may be helpful to sharpen employees' awareness of leisure thoughts by explaining this phenomenon and providing information about the frequencies. In summary, recovery training and work engagement could benefit from considering leisure thoughts and upcoming leisure time and activities.

\section{Limitations and Future Research}

This dissertation is not without limitations, which point the way out to future research. First, the overarching goal was to investigate the anticipation of leisure time as an upcoming resource gain. Across all three studies, employees indicated high pleasant anticipation of their planned leisure activities. Furthermore, most employees mentioned positive planned leisure activities, even though they were not asked to focus on positive leisure activities. In line with the positive leisure activities, general anticipation of leisure time was on average positive. However, leisure time may also contain negative leisure activities. Thus, although beyond the scope of this dissertation, these negative leisure activities may indicate upcoming resource losses, which may also affect work engagement and the frequency of leisure thoughts. Because pleasant anticipation refers to positive, upcoming events, it cannot assess anticipated resource losses. Therefore, future research could develop a new questionnaire to measure anticipated resources losses and gains because of planned leisure activities. This questionnaire may help investigate anticipated resource losses and examine whether positive and negative planned leisure activities interact. 
Second, each diary study contained several daily or hourly questionnaires, and the focal constructs and work engagement were measured in separate questionnaires. Thus, the predictor variables were assessed before the outcome variable ${ }^{12}$. Furthermore, because within-person effects refer to fluctuations within the same person, many variables (i.e., between-person differences) could be excluded as alternative explanations (e.g., personality traits, different organisations) for the within-person results. Therefore, the study design may be a tentative hint for causality, yet an experimental design is necessary to demonstrate that the anticipation of leisure time is the reason for changes in work engagement and leisure thoughts. Because leisure activities have different values for different employees, manipulating leisure activities to affect the anticipated resource gain may be difficult. However, future research could use recovery training to study the relationship between pleasant anticipation and work engagement. In a first step, employees could summarize various leisure activities and rate their value. In a second step, as part of the recovery training, researchers could use the individual ratings to instruct participants to engage in high valued leisure activities on one day and in low valued leisure activities on another day to manipulate the anticipated resource gain. To examine within-person differences, work engagement from these different days could be compared. Moreover, an experimental group could be contrasted with a control group to study differences between persons. While employees in the experimental group could rate their pleasant anticipation, employees in the control group could not be asked about upcoming leisure activities. However, it is important to note that the control group design only answers whether the measurement of pleasant anticipation (i.e., asking for planned leisure activities) may be responsible for changes in work engagement.

Third, because work engagement is an essential construct for employees and organisations (for a review, see Bailey et al., 2017), it was investigated in all three studies. Work engagement is associated with employees' task performance (for a meta-analysis, see Christian et al., 2011), but future research could investigate the relationship between pleasant anticipation and general anticipation of leisure time and task performance. Furthermore, other work-related outcomes could be considered, for instance, organisational citizenship behaviour (OCB; Organ, 1988, 2018). OCB is defined as positive employee behaviour that is beneficial for the organisation and not part of the formal job description (e.g., helping co-workers, speaking good about the organisation). Because positive affect is one antecedent of OCB (e.g.,

\footnotetext{
${ }^{12}$ Answering the question about the relationship between leisure thoughts and work engagement within the same hour in study 2 is the only exception when the predictors and the outcomes were measured simultaneously.
} 
Spector \& Fox, 2002; Ziegler et al., 2012), the positive affective reaction pleasant anticipation may also be related to OCB. Thus, future research could broaden the understanding of the anticipation of leisure time by investigating further consequences of pleasant anticipation of a planned leisure activity.

Fourth, work engagement, pleasant anticipation, leisure thoughts, and general anticipation were assessed with self-ratings. Therefore, some part of the variance in work engagement may be explained by using the same method (i.e., common-method variance; Podsakoff et al., 2012). However, all focal constructs included in this dissertation could only be assessed with self-rating, as they refer to employees' affective reactions (pleasant anticipation), individual evaluations (general anticipation of leisure time), frequencies of thoughts (ToPLA/ToLT), and an internal, motivational state (work engagement). Furthermore, the independent and the dependent variable were measured at different time points and with different scales (i.e., Kunin-scale for general anticipation, an abstract rating scale for leisure thoughts) reducing common-method variance (Podsakoff et al., 2003). Nevertheless, future research could use external ratings to further decrease common-method variance. For instance, supervisors could indicate employees' task performance, colleagues could rate OCB and counterproductive work behaviour, or customers' satisfaction could be measured. Moreover, in a laboratory setting, the distraction caused by leisure thoughts could be operationalized with electroencephalography (for the use in organisational research, see Tivadar \& Murray, 2019; Waldman et al., 2019) or eye tracking (for the use in organisational research, see Meißner \& Oll, 2019).

Finally, based on COR theory (Hobfoll, 1989; Hobfoll et al., 2018), I hypothesized that anticipated resource gains are positively related to employees' work engagement and that pleasant anticipation and general anticipation indicate these anticipated resource gains. However, whether the anticipated resource gains from leisure time concurs with the experienced resource gains the subsequent day was not investigated. Therefore, it remains unclear whether pleasant anticipation and general anticipation are the best possible indicators of upcoming resource gains. Furthermore, I assumed that employees invest more resources into their work engagement when they anticipate a resource gain during leisure time. One may argue that employees' implicit theories about resources and anticipated resource gains are crucial for this assumption (for implicit theories about will-power, see Job et al., 2010). Thus, employees must believe that resources become exhausted during the working day and that leisure time and leisure activities provide opportunities to (re)gain resources. A laboratory experiment may be one way to examine anticipated resource gains in more detail. For instance, participants could 
solve different resource-depleting tasks for 60 minutes, and one group is promised a 10minutes-break after 30 minutes (i.e., an anticipated resource gain), while no break is announced in the other group. According to COR theory (Hobfoll, 1989; Hobfoll et al., 2018), employees in the break condition should anticipate a resource gain and be more engaged in the first 30 minutes of the experiments. In addition, a questionnaire could be developed to measure employees' implicit theories about anticipated resources gains and used in this experiment to examine interindividual differences.

\section{General Conclusion}

Leisure time is a crucial part of the working day and provides various opportunities to recover and gain new resources. Until now, research has focused on the beneficial characteristics of past leisure time and leisure activities, for instance, as one explanation for high work engagement. This dissertation expanded this previous view and demonstrated that upcoming leisure time and leisure activities are also important to understand employees' work engagement. Employees think about and anticipate upcoming leisure time and leisure activities, which has positive and negative consequences. Therefore, this dissertation is the first hint that anticipation of leisure time should also be considered in future research when explaining employees' feelings, cognitions, and behaviour at work. 


\section{REFERENCES}

Aguinis, H., Gottfredson, R. K., \& Culpepper, S. A. (2013). Best-Practice Recommendations for Estimating Cross-Level Interaction Effects Using Multilevel Modeling. Journal of Management, 39(6), 1490-1528. https://doi.org/10.1177/0149206313478188

Areni, C. S. (2008). (Tell me why) I don't like Mondays: Does an overvaluation of future discretionary time underlie reported weekly mood cycles? Cognition \& Emotion, 22(7), 1228-1252. https://doi.org/10.1080/02699930701686107

Areni, C. S., Burger, M., \& Zlatevska, N. (2011). Factors affecting the extent of Monday blues: Evidence from a meta-analysis. Psychological Reports, 109(3), 723-733. https://doi.org/10.2466/13.20.PR0.109.6.723-733

Aspinwall, L. G. (2005). The psychology of future-oriented thinking: From achievement to proactive coping, adaptation, and aging. Motivation and Emotion, 29(4), 203-235. https://doi.org/10.1007/s11031-006-9013-1

Aspinwall, L. G., \& Taylor, S. E. (1997). A stitch in time: Self-regulation and proactive coping. Psychological Bulletin, 121(3), 417-436. https://doi.org/10.1037//00332909.121.3.417

Atance, C. M., \& O'Neill, D. K. (2001). Episodic future thinking. Trends in Cognitive Sciences, 5(12), 533-539. https://doi.org/10.1016/S1364-6613(00)01804-0

Baethge, A., \& Rigotti, T. (2013). Interruptions to workflow: Their relationship with irritation and satisfaction with performance, and the mediating roles of time pressure and mental demands. Work \& Stress, 27(1), 43-63. https://doi.org/10.1080/02678373.2013.761783

Bagozzi, R. P., Baumgartner, H., \& Pieters, R. (1998). Goal-directed emotions. Cognition \& Emotion, 12(1), 1-26. https://doi.org/10.1080/026999398379754

Bailey, C., Madden, A., Alfes, K., \& Fletcher, L. (2017). The Meaning, Antecedents and Outcomes of Employee Engagement: A Narrative Synthesis. International Journal of Management Reviews, 19(1), 31-53. https://doi.org/10.1111/ijmr.12077 
Bakker, A. B., \& Demerouti, E. (2017). Job demands-resources theory: Taking stock and looking forward. Journal of Occupational Health Psychology, 22(3), 273-285. https://doi.org/10.1037/ocp0000056

Bakker, A. B., Demerouti, E., \& Sanz-Vergel, A. I. (2014). Burnout and Work Engagement: The JD-R Approach. Annual Review of Organizational Psychology and Organizational Behavior, 1(1), 389-411. https://doi.org/10.1146/annurev-orgpsych031413-091235

Bakker, A. B., Schaufeli, W. B., Leiter, M. P., \& Taris, T. W. (2008). Work engagement: An emerging concept in occupational health psychology. Work \& Stress, 22(3), 187-200. https://doi.org/10.1080/02678370802393649

Bandura, A. (1977). Self-efficacy: Toward a unifying theory of behavioral change. Psychological Review, 84(2), 191-215. https://doi.org/10.1037/0033-295X.84.2.191

Bandura, A. (1994). Self-efficacy. In V. S. Ramachaudran (Ed.), Encyclopedia of human behavior (Vol. 4, pp. 71-81). Academic Press.

Bandura, A. (Ed.). (1995). Self-efficacy in changing societies (1st ed.). Cambridge University.

Barber, L., Grawitch, M. J., \& Munz, D. C. (2013). Are better sleepers more engaged workers? A self-regulatory approach to sleep hygiene and work engagement. Stress and Health: Journal of the International Society for the Investigation of Stress, 29(4), 307-316. https://doi.org/10.1002/smi.2468

Barsics, C., van der Linden, M., \& D'Argembeau, A. (2016). Frequency, characteristics, and perceived functions of emotional future thinking in daily life. Quarterly Journal of Experimental Psychology (2006), 69(2), 217-233. https://doi.org/10.1080/17470218.2015.1051560

Baumeister, R. F., Vohs, K. D., DeWall, C. N., \& Zhang, L. (2007). How emotion shapes behavior: Feedback, anticipation, and reflection, rather than direct causation. Personality and Social Psychology Review, 11(2), 167-203. https://doi.org/10.1177/1088868307301033

Baumeister, R. F., Vohs, K. D., \& Oettingen, G. (2016). Pragmatic prospection: How and why people think about the future. Review of General Psychology, 20(1), 3-16. https://doi.org/10.1037/gpr0000060

Baumgartner, H., Pieters, R., \& Bagozzi, R. P. (2008). Future-oriented emotions: Conceptualization and behavioral effects. European Journal of Social Psychology, 38(4), 685-696. https://doi.org/10.1002/ejsp.467 
Beal, D. J., Weiss, H. M., Barros, E., \& MacDermid, S. M. (2005). An episodic process model of affective influences on performance. Journal of Applied Psychology, 90(6), 1054-1068. https://doi.org/10.1037/0021-9010.90.6.1054

Becker, T. E. (2005). Potential problems in the statistical control of variables in organizational research: A qualitative analysis with recommendations. Organizational Research Methods, 8(3), 274-289. https://doi.org/10.1177/1094428105278021

Bennett, A. A., Bakker, A. B., \& Field, J. G. (2018). Recovery from work-related effort: A meta-analysis. Journal of Organizational Behavior, 39(3), 262-275. https://doi.org/10.1002/job.2217

Besser, A., Zeigler-Hill, V., Weinberg, M., \& Pincus, A. L. (2016). Do great expectations lead to great disappointments? Pathological narcissism and the evaluation of vacation experiences. Personality and Individual Differences, 89(Supplement C), 75-79. https://doi.org/10.1016/j.paid.2015.10.003

Binnewies, C., Sonnentag, S., \& Mojza, E. J. (2009). Daily performance at work: feeling recovered in the morning as a predictor of day-level job performance. Journal of Organizational Behavior, 30(1), 67-93. https://doi.org/10.1002/job.541

Bliese, P. D., Edwards, J. R., \& Sonnentag, S. (2017). Stress and well-being at work: A century of empirical trends reflecting theoretical and societal influences. Journal of Applied Psychology, 102(3), 389-402. https://doi.org/10.1037/ap10000109

Bliese, P. D., \& Ployhart, R. E. (2002). Growth modeling using random coefficient models: Model building, testing, and illustrations. Organizational Research Methods, 5(4), 362-387. https://doi.org/10.1177/109442802237116

Bosch, C., Sonnentag, S., \& Pinck, A. S. (2018). What makes for a good break? A diary study on recovery experiences during lunch break. Journal of Occupational and Organizational Psychology, 91(1), 134-157. https://doi.org/10.1111/joop.12195

Bradley, M. M., \& Lang, P. J. (1994). Measuring emotion: The self-assessment manikin and the semantic differential. Journal of Behavior Therapy and Experimental Psychiatry, 25(1), 49-59. https://doi.org/10.1016/0005-7916(94)90063-9

Brajša-Žganec, A., Merkaš, M., \& Šverko, I. (2011). Quality of life and leisure activities: How do leisure activities contribute to subjective well-being? Social Indicators Research, 102(1), 81-91. https://doi.org/10.1007/s11205-010-9724-2

Breevaart, K., Bakker, A. B., Derks, D., \& van Vuuren, T. C. V. (2020). Engagement during demanding workdays: A diary study on energy gained from off-job activities. 
International Journal of Stress Management, 27(1), 45-52.

https://doi.org/10.1037/str0000127

Breevaart, K., Bakker, A., Hetland, J., Demerouti, E., Olsen, O. K., \& Espevik, R. (2014).

Daily transactional and transformational leadership and daily employee engagement. Journal of Occupational and Organizational Psychology, 87(1), 138-157. https://doi.org/10.1111/joop.12041

Bruininks, P., \& Malle, B. F. (2005). Distinguishing hope from optimism and related affective states. Motivation and Emotion, 29(4), 324-352. https://doi.org/10.1007/s11031-0069010-4

Bryant, F. B. (2003). Savoring beliefs inventory (SBI): A scale for measuring beliefs about savouring. Journal of Mental Health, 12(2), 175-196. https://doi.org/10.1080/0963823031000103489

Bryant, F. B., \& Veroff, J. (2006a). Savoring: A new model of positive experience. Taylor \& Francis.

Bryant, F. B., \& Veroff, J. (2006b). Savoring: A new model of positive experience. Taylor and Fracis.

Buysse, D. J., Reynolds III, C. F., Monk, T. H., Berman, S. R., \& Kupfer, D. J. (1989). The pittsburgh sleep quality index. Psychiatry Research, 28(2), 193-213. https://doi.org/10.1016/0165-1781(89)90047-4

Byrne, Z. S., Peters, J. M., \& Weston, J. W. (2016). The struggle with employee engagement: Measures and construct clarification using five samples. Journal of Applied Psychology, 101(9), 1201-1227. https://doi.org/10.1037/ap10000124

Carrera, P., Caballero, A., \& Muñoz, D. (2012). Future-oriented emotions in the prediction of binge-drinking intention and expectation: The role of anticipated and anticipatory emotions. Scandinavian Journal of Psychology, 53(3), 273-279. https://doi.org/10.1111/j.1467-9450.2012.00948.x

Casper, A., \& Sonnentag, S. (2020). Feeling exhausted or vigorous in anticipation of high workload? The role of worry and planning during the evening. Journal of Occupational and Organizational Psychology, 93(1), 215-242. https://doi.org/10.1111/joop.12290

Casper, A., Sonnentag, S., \& Tremmel, S. (2017). Mindset matters: the role of employees' stress mindset for day-specific reactions to workload anticipation. European Journal of Work and Organizational Psychology, 26(6), 798-810. https://doi.org/10.1080/1359432X.2017.1374947 
Chawla, N., MacGowan, R. L., Gabriel, A. S., \& Podsakoff, N. P. (2020). Unplugging or staying connected? Examining the nature, antecedents, and consequences of profiles of daily recovery experiences. Journal of Applied Psychology, 105(1), 19-39. https://doi.org/10.1037/ap10000423

Chen, S., Westman, M., \& Eden, D. (2009). Impact of enhanced resources on anticipatory stress and adjustment to new information technology: A field-experimental test of conservation of resources theory. Journal of Occupational Health Psychology, 14(3), 219-230. https://doi.org/10.1037/a0015282

Christian, M. S., Garza, A. S., \& Slaughter, J. E. (2011). Work engagement: A quantitative review and test of its relations with task and contextual performance. Personnel Psychology, 64(1), 89-136. https://doi.org/10.1111/j.1744-6570.2010.01203.x

Chu, S., Handley, V., \& Cooper, S. R. (2003). Eliminating context-dependent forgetting: Changing contexts can be as effective as reinstating them. The Psychological Record, 53(4), 549-559. https://doi.org/10.1007/BF03395452

Crawford, E. R., Lepine, J. A., \& Rich, B. L. (2010). Linking job demands and resources to employee engagement and burnout: A theoretical extension and meta-analytic test. Journal of Applied Psychology, 95(5), 834-848. https://doi.org/10.1037/a0019364

D’Argembeau, A. (2018). Mind-wandering and self-referential thought. In K. C. R. Fox \& K. Christoff (Eds.), The oxford handbook of spontaneous thought: Mind-wandering, creativity, and dreaming (pp. 181-192). Oxford University.

Dane, E. (2018). Where is my mind? Theorizing mind wandering and its performance-related consequences in organizations. Academy of Management Review, 43(2), 179-197. https://doi.org/10.5465/amr.2015.0196

D'Argembeau, A., Renaud, O., \& van der Linden, M. (2011). Frequency, characteristics and functions of future-oriented thoughts in daily life. Applied Cognitive Psychology, 25(1), 96-103. https://doi.org/10.1002/acp.1647

de Bloom, J., Rantanen, J., Tement, S., \& Kinnunen, U. (2018). Longitudinal leisure activity profiles and their associations with recovery experiences and job Performance. Leisure Sciences, 40(3), 151-173. https://doi.org/10.1080/01490400.2017.1356254

Demerouti, E., Bakker, A. B., Geurts, S. A. E., \& Taris, T. W. (2009). Daily recovery from work-related effort during non-work time. In S. Sonnentag, P. L. Perrewé, \& D. C. Ganster (Eds.), Current perspectives on job-stress recovery: Research in occupational stress and well being (pp. 85-124). Emerald Group. 
Demerouti, E., \& Peeters, M. C. W. (2018). Transmission of reduction-oriented crafting among colleagues: A diary study on the moderating role of working conditions. Journal of Occupational and Organizational Psychology, 91(2), 209-234. https://doi.org/10.1111/joop.12196

Diehl, M., Semegon, A. B., \& Schwarzer, R. (2006). Assessing attention control in goal pursuit: A component of dispositional self-regulation. Journal of Personality Assessment, 86(3), 306-317. https://doi.org/10.1207/s15327752jpa8603_06

Dimitrova, N. G., van Dyck, C., van Hooft, E. A. J., \& Groenewegen, P. (2015). Don't fuss, focus: The mediating effect of on-task thoughts on the relationship between error approach instructions and task performance. Applied Psychology: An International Review, 64(3), 599-624. https://doi.org/10.1111/apps.12029

DiStaso, M. J., \& Shoss, M. K. (2020). Looking forward: How anticipated workload change influences the present workload-emotional strain relationship. Journal of Occupational Health Psychology, 25(6), 401-409. https://doi.org/10.1037/ocp0000261

Dumas, T. L., \& Perry-Smith, J. E. (2018). The paradox of family structure and plans after work: Why single childless employees may be the least absorbed at work. Academy of Management Journal, 61(4), 1231-1252. https://doi.org/10.5465/amj.2016.0086

Ebert, D. D., Berking, M., Thiart, H., Riper, H., Laferton, J. A. C., Cuijpers, P., Sieland, B., \& Lehr, D. (2015). Restoring depleted resources: Efficacy and mechanisms of change of an internet-based unguided recovery training for better sleep and psychological detachment from work. Health Psychology, 34S, 1240-1251. https://doi.org/10.1037/hea0000277

Flaxman, P. E., Stride, C. B., Söderberg, M., Lloyd, J., Guenole, N., \& Bond, F. W. (2018). Relationships between two dimensions of employee perfectionism, postwork cognitive processing, and work day functioning. European Journal of Work and Organizational Psychology, 27(1), 56-69. https://doi.org/10.1080/1359432X.2017.1391792

Ford, M. T., Matthews, R. A., Wooldridge, J. D., Mishra, V., Kakar, U. M., \& Strahan, S. R. (2014). How do occupational stressor-strain effects vary with time? A review and meta-analysis of the relevance of time lags in longitudinal studies. Work \& Stress, 28(1), 9-30. https://doi.org/10.1080/02678373.2013.877096

Fritz, C., \& Demsky, C. A. (2019). Non-work time as individual resource building: A review and research agenda. In R. J. Burke \& A. M. Richardsen (Eds.), Creating psychologically healthy workplaces (pp. 133-151). Edward Elgar Publishing. 
Fritz, C., \& Sonnentag, S. (2005). Recovery, health, and job performance: Effects of weekend experiences. Journal of Occupational Health Psychology, 10(3), 187-199. https://doi.org/10.1037/1076-8998.10.3.187

Fritz, C., \& Sonnentag, S. (2006). Recovery, well-being, and performance-related outcomes: The role of workload and vacation experiences. Journal of Applied Psychology, 91(4), 936-945. https://doi.org/10.1037/0021-9010.91.4.936

Gardner, D. G., Cummings, L. L., Dunham, R. B., \& Pierce, J. L. (1998). Single-item versus multiple-item measurement scales: An empirical comparison. Educational and Psychological Measurement, 58(6), 898-915. https://doi.org/10.1177/0013164498058006003

Gardner, D. G., Dunham, R. B., Cummings, L. L., \& Pierce, J. L. (1987). Employee focus of attention and reactions to organizational change. The Journal of Applied Behavioral Science, 23(3), 351-370. https://doi.org/10.1177/002188638702300305

Gardner, D. G., Dunham, R. B., Cummings, L. L., \& Pierce, J. L. (1989). Focus of attention at work: Construct definition and empirical validation. Journal of Occupational Psychology, 62(1), 61-77. https://doi.org/10.1111/j.2044-8325.1989.tb00478.x

Garrick, A., Mak, A. S., Cathcart, S., Winwood, P. C., Bakker, A. B., \& Lushington, K. (2014). Psychosocial safety climate moderating the effects of daily job demands and recovery on fatigue and work engagement. Journal of Occupational and Organizational Psychology, 87(4), 694-714. https://doi.org/10.1111/joop.12069

Gebauer, J. E., Riketta, M., Broemer, P., \& Maio, G. R. (2008). “How much do you like your name?" An implicit measure of global self-esteem. Journal of Experimental Social Psychology, 44(5), 1346-1354. https://doi.org/10.1016/j.jesp.2008.03.016

Geurts, S. A. E., \& Demerouti, E. (2003). Work/non-work interface: A review of theories and findings. In Schabracq, J., Marc, J. A. Winnubst, \& Cooper, L., Cary (Eds.), The handbook of work and health psychology (Vol. 2, pp. 279-312). Wiley and Sons.

Goering, D. D., Shimazu, A., Zhou, F., Wada, T., \& Sakai, R. (2017). Not if, but how they differ: A meta-analytic test of the nomological networks of burnout and engagement. Burnout Research, 5, 21-34. https://doi.org/10.1016/j.burn.2017.05.003

Gollwitzer, P. M. (1996). Benefits of planning. In P. M. Gollwitzer \& J. A. Bargh (Eds.), The psychology of action: Linking cognition and motivation to behavior (pp. 287-312). Guilford. 
Gorgievski, M. J., Halbesleben, J. R. B., \& Bakker, A. B. (2011). Expanding the boundaries of psychological resource theories. Journal of Occupational and Organizational Psychology, 84(1), 1-7. https://doi.org/10.1111/j.2044-8325.2010.02015.x

Gorgievski, M. J., \& Hobfoll, S. E. (2008a). Work can burn us out or fire us up: Conservation of resources in burnout and engagement. In J. R. B. Halbesleben (Ed.), Handbook of stress and burnout in health care (pp. 7-22). Nova Science.

Gorgievski, M. J., \& Hobfoll, S. E. (2008b). Work can burn us out or fire us up: Conservation of resources in burnout and engagement. In J. R. B. Halbesleben (Ed.), Handbook of stress and burnout in health care (pp. 7-22). Nova Science.

Graham, L. E., Thomson, A. L., Nakamura, J., Brandt, I. A., \& Siegel, J. T. (2019). Finding a family: A categorization of enjoyable emotions. The Journal of Positive Psychology, 14(2), 206-229. https://doi.org/10.1080/17439760.2017.1402074

Hahn, V. C., Binnewies, C., Sonnentag, S., \& Mojza, E. J. (2011). Learning how to recover from job stress: Effects of a recovery training program on recovery, recovery-related self-efficacy, and well-being. Journal of Occupational Health Psychology, 16(2), 202-216. https://doi.org/10.1037/a0022169

Hakanen, J. J., \& Schaufeli, W. B. (2012). Do burnout and work engagement predict depressive symptoms and life satisfaction? A three-wave seven-year prospective study. Journal of Affective Disorders, 141(2-3), 415-424. https://doi.org/10.1016/j.jad.2012.02.043

Hakanen, J. J., Schaufeli, W. B., \& Ahola, K. (2008). The Job Demands-Resources model: A three-year cross-lagged study of burnout, depression, commitment, and work engagement. Work \& Stress, 22(3), 224-241. https://doi.org/10.1080/02678370802379432

Halbesleben, J. R. B. (2010a). A meta-analysis of work engagement: Relationship with burnout, demands, resources and consequences. In M. P. Leiter \& A. B. Bakker (Eds.), Work engagement: A handbook of essential theory and research (pp. 102-117). Taylor and Fracis.

Halbesleben, J. R. B. (2010b). A meta-analysis of work engagement: Relationship with burnout, demands, resources and sonsequences. In M. P. Leiter \& A. B. Bakker (Eds.), Work engagement: A handbook of essential theory and research (pp. 102-117). Taylor \& Francis.

Halbesleben, J. R. B., Neveu, J.-P., Paustian-Underdahl, S. C., \& Westman, M. (2014). Getting to the "COR": Understanding the role of resources in conservation of 
resources theory. Journal of Management, 40(5), 1334-1364.

https://doi.org/10.1177/0149206314527130

Harris, R. B., Harris, K. J., \& Harvey, P. (2007). A test of competing models of the relationships among perceptions of organizational politics, perceived organizational support, and individual outcomes. The Journal of Social Psychology, 147(6), 631-655. https://doi.org/10.3200/SOCP.147.6.631-656

Hobfoll, S. E. (1989). Conservation of resources: A new attempt at conceptualizing stress. American Psychologist, 44(3), 513-524. https://doi.org/10.1037/0003-066X.44.3.513

Hobfoll, S. E. (2001a). The influence of culture, community, and the nested-self in the stress process: Advancing conservation of resources theory. Applied Psychology: An International Review, 50(3), 337-421. https://doi.org/10.1111/1464-0597.00062

Hobfoll, S. E. (2001b). The influence of culture, community, and the nested-self in the stress process: Advancing conservation of resources theory. Applied Psychology: An International Review, 50(3), 337-421. https://doi.org/10.1111/1464-0597.00062

Hobfoll, S. E., Halbesleben, J. R. B., Neveu, J.-P., \& Westman, M. (2018). Conservation of resources in the organizational context: The reality of resources and their consequences. Annual Review of Organizational Psychology and Organizational Behavior, 5(1), 103-128. https://doi.org/10.1146/annurev-orgpsych-032117-104640

Hobfoll, S. E., Johnson, R. J., Ennis, N., \& Jackson, A. P. (2003). Resource loss, resource gain, and emotional outcomes among inner city women. Journal of Personality and Social Psychology, 84(3), 632-643. https://doi.org/10.1037/0022-3514.84.3.632

Howard, M. W., \& Kahana, M. J. (1999). Contextual variability and serial position effects in free recall. Journal of Experimental Psychology: Learning, Memory, and Cognition, 25(4), 923-941. https://doi.org/10.1037/0278-7393.25.4.923

Hu, Q., Schaufeli, W. B., \& Taris, T. W. (2017). How are changes in exposure to job demands and job resources related to burnout and engagement? A longitudinal study among Chinese nurses and police officers. Stress and Health, 33(5), 631-644. https://doi.org/10.1002/smi.2750

Hülsheger, U. R. (2016). From dawn till dusk: Shedding light on the recovery process by investigating daily change patterns in fatigue. Journal of Applied Psychology, 101(6), 905-914. https://doi.org/10.1037/ap10000104

Hunter, E. M., \& Wu, C. (2016). Give me a better break: Choosing workday break activities to maximize resource recovery. Journal of Applied Psychology, 101(2), 302-311. https://doi.org/10.1037/ap10000045 
Job, V., Dweck, C. S., \& Walton, G. M. (2010). Ego depletion-is it all in your head? Implicit theories about willpower affect self-regulation. Psychological Science, 21(11), 16861693. https://doi.org/10.1177/0956797610384745

Kabanoff, B. (1980). Work and nonwork: A review of models, methods, and findings. Psychological Bulletin, 88(1), 60-77. https://doi.org/10.1037/0033-2909.88.1.60

Kahn, W. A. (1990). Psychological conditions of personal engagement and disengagement at work. Academy of Management Journal, 33(4), 692-724. https://doi.org/10.2307/256287

Kanfer, R., \& Ackerman, P. L. (1989). Motivation and cognitive abilities: An integrative/aptitude-treatment interaction approach to skill acquisition. Journal of Applied Psychology, 74(4), 657-690. https://doi.org/10.1037//0021-9010.74.4.657

Kanfer, R., Ackerman, P. L., Murtha, T. C., Dugdale, B., \& al, e. (1994). Goal setting, conditions of practice, and task performance: A resource allocation perspective. Journal of Applied Psychology, 79(6), 826-835. https://doi.org/10.1037//00219010.79.6.826

Kessler, E.-M., \& Staudinger, U. M. (2009). Affective experience in adulthood and old age: The role of affective arousal and perceived affect regulation. Psychology and Aging, 24(2), 349-362. https://doi.org/10.1037/a0015352

King, L. A. (2001). The health benefits of writing about life goals. Personality \& Social Psychology Bulletin, 27(7), 798-807. https://doi.org/10.1177/0146167201277003

Klinger, E. (2013). Goal commitments and the content of thoughts and dreams: Basic principles. Frontiers in Psychology, 4, 415. https://doi.org/10.3389/fpsyg.2013.00415

Knight, C., Patterson, M., \& Dawson, J. (2019). Work engagement interventions can be effective: a systematic review. European Journal of Work and Organizational Psychology, 28(3), 348-372. https://doi.org/10.1080/1359432X.2019.1588887

Koenig-Lewis, N., \& Palmer, A. (2014). The effects of anticipatory emotions on service satisfaction and behavioral intention. Journal of Services Marketing, 28(6), 437-451. https://doi.org/10.1108/JSM-09-2013-0244

Kong, D. T., Tuncel, E., \& McLean Parks, J. (2011a). Anticipating happiness in a future negotiation: anticipated happiness, propensity to initiate a negotiation, and individual outcomes. Negotiation and Conflict Management Research, 4(3), 219-247. https://doi.org/10.1111/j.1750-4716.2011.00081.x

Kong, D. T., Tuncel, E., \& McLean Parks, J. (2011b). Anticipating happiness in a future negotiation: Anticipated happiness, propensity to initiate a negotiation, and individual 
outcomes. Negotiation and Conflict Management Research, 4(3), 219-247. https://doi.org/10.1111/j.1750-4716.2011.00081.x

Kühnel, J., Sonnentag, S., \& Bledow, R. (2012). Resources and time pressure as day-level antecedents of work engagement. Journal of Occupational and Organizational Psychology, 85(1), 181-198. https://doi.org/10.1111/j.2044-8325.2011.02022.x

Kühnel, J., Sonnentag, S., Bledow, R., \& Melchers, K. G. (2018). The relevance of sleep and circadian misalignment for procrastination among shift workers. Journal of Occupational and Organizational Psychology, 91(1), 110-133. https://doi.org/10.1111/joop.12191

Kühnel, J., Zacher, H., de Bloom, J., \& Bledow, R. (2017). Take a break! Benefits of sleep and short breaks for daily work engagement. European Journal of Work and Organizational Psychology, 26(4), 481-491. https://doi.org/10.1080/1359432X.2016.1269750

Kunin, T. (1955). The construction of a new type of attitude measure. Personnel Psychology, 8(1), 65-77. https://doi.org/10.1111/j.1744-6570.1955.tb01189.x

Larsen, R. J., \& Kasimatis, M. (1990). Individual differences in entrainment of mood to the weekly calendar. Journal of Personality and Social Psychology, 58(1), 164-171. https://doi.org/10.1037/0022-3514.58.1.164

Leroy, S. (2009). Why is it so hard to do my work? The challenge of attention residue when switching between work tasks. Organizational Behavior and Human Decision Processes, 109(2), 168-181. https://doi.org/10.1016/j.obhdp.2009.04.002

Leroy, S., \& Glomb, T. M. (2018a). Tasks interrupted: How anticipating time pressure on resumption of an interrupted task causes attention residue and low performance on interrupting tasks and how a "ready-to-resume" plan mitigates the effects. Organization Science, 29(3), 380-397. https://doi.org/10.1287/orsc.2017.1184

Leroy, S., \& Glomb, T. M. (2018b). Tasks Interrupted: How Anticipating Time Pressure on Resumption of an Interrupted Task Causes Attention Residue and Low Performance on Interrupting Tasks and How a "Ready-to-Resume" Plan Mitigates the Effects. Organization Science, 29(3), 380-397. https://doi.org/10.1287/orsc.2017.1184

Lesener, T., Gusy, B., \& Wolter, C. (2019). The job demands-resources model: A metaanalytic review of longitudinal studies. Work \& Stress, 33(1), 76-103. https://doi.org/10.1080/02678373.2018.1529065

Liberman, N., \& Trope, Y. (1998). The role of feasibility and desirability considerations in near and distant future decisions: A test of temporal construal theory. Journal of 
Personality and Social Psychology, 75(1), 5-18. https://doi.org/10.1037//00223514.75.1.5

Lischetzke, T., Reis, D., \& Arndt, C. (2015). Data-analytic strategies for examining the effectiveness of daily interventions. Journal of Occupational and Organizational Psychology, 88(3), 587-622. https://doi.org/10.1111/joop.12104

Liu, H., Ji, Y., \& Dust, S. B. (2020). "Fully recharged" evenings? The effect of evening cyber leisure on next-day vitality and performance through sleep quantity and quality, bedtime procrastination, and psychological detachment, and the moderating role of mindfulness. Journal of Applied Psychology. Advance online publication. https://doi.org/10.1037/ap10000818

Loewenstein, G. (1987). Anticipation and the valuation of delayed consumption. The Economic Journal, 97(387), 666-684. https://doi.org/10.2307/2232929

Maglio, S. J., \& Polman, E. (2016). Revising probability estimates: Why increasing likelihood means increasing impact. Journal of Personality and Social Psychology, 111(2), 141158. https://doi.org/10.1037/pspa0000058

Marchetti, I., Koster, E. H. W., Klinger, E., \& Alloy, L. B. (2016). Spontaneous thought and vulnerability to mood disorders: The dark side of the wandering mind. Clinical Psychological Science, 4(5), 835-857. https://doi.org/10.1177/2167702615622383

Martin, L. L., \& Tesser, A. (1996). Some ruminative thoughts. In R. S. Wyer (Ed.), Advances in social cognition (pp. 1-48). Lawrence Erlbaum.

Maslach, C., Schaufeli, W. B., \& Leiter, M. P. (2001). Job burnout. Annual Review of Psychology, 52, 397-422. https://doi.org/10.1146/annurev.psych.52.1.397

Mason, M. F., \& Reinholtz, N. (2015). Avenues down which a self-reminding mind can wander. Motivation Science, 1(1), 1-21. https://doi.org/10.1037/mot0000011

Mazzola, J. J., \& Disselhorst, R. (2019). Should we be “challenging” employees? A critical review and meta-analysis of the challenge-hindrance model of stress. Journal of Organizational Behavior, 40(8), 949-961. https://doi.org/10.1002/job.2412

McCormick, B. W., Reeves, C. J., Downes, P. E., Li, N., \& Ilies, R. (2018). Scientific contributions of within-person research in management: Making the juice worth the squeeze. Journal of Management, 1, 014920631878843. https://doi.org/10.1177/0149206318788435

Meijman, F. T., \& Mulder, G. (1998). Psychological aspects of workload. In P. J. D. Drenth, H. Thierry, \& C. J. de Wolff (Eds.), A handbook of work and organizational psychology (2nd ed., Vol. 2, pp. 5-33). Taylor \& Francis. 
Meißner, M., \& Oll, J. (2019). The promise of eye-tracking methodology in organizational research: A taxonomy, review, and future avenues. Organizational Research Methods, 22(2), 590-617. https://doi.org/10.1177/1094428117744882

Merlo, K. L., Shaughnessy, S. P., \& Weiss, H. M. (2018). Affective influences on withinperson changes in work performance as mediated by attentional focus. European Journal of Work and Organizational Psychology, 27(1), 126-139. https://doi.org/10.1080/1359432X.2017.1417258

Miranda, R., Wheeler, A., Polanco-Roman, L., \& Marroquín, B. (2017). The future-oriented repetitive thought (FoRT) scale: A measure of repetitive thinking about the future. Journal of Affective Disorders, 207, 336-345. https://doi.org/10.1016/j.jad.2016.09.055

Mitchell, T. R., Thompson, L., Peterson, E., \& Cronk, R. (1997). Temporal adjustments in the evaluation of events: The "rosy view". Journal of Experimental Social Psychology, 33(4), 421-448. https://doi.org/10.1006/jesp.1997.1333

Mojza, E. J., Lorenz, C., Sonnentag, S., \& Binnewies, C. (2010). Daily recovery experiences: The role of volunteer work during leisure time. Journal of Occupational Health Psychology, 15(1), 60-74. https://doi.org/10.1037/a0017983

Monfort, S. S., Stroup, H. E., \& Waugh, C. E. (2015). The impact of anticipating positive events on responses to stress. Journal of Experimental Social Psychology, 58, 11-22. https://doi.org/10.1016/j.jesp.2014.12.003

Mooneyham, B. W., \& Schooler, J. W. (2013). The costs and benefits of mind-wandering: A review. Canadian Journal of Experimental Psychology = Revue Canadienne De Psychologie Experimentale, 67(1), 11-18. https://doi.org/10.1037/a0031569

Mrazek, M. D., Chin, J. M., Schmader, T., Hartson, K. A., Smallwood, J., \& Schooler, J. W. (2011). Threatened to distraction: Mind-wandering as a consequence of stereotype threat. Journal of Experimental Social Psychology, 47(6), 1243-1248. https://doi.org/10.1016/j.jesp.2011.05.011

Mrazek, M. D., Smallwood, J., Franklin, M. S., Chin, J. M., Baird, B., \& Schooler, J. W. (2012). The role of mind-wandering in measurements of general aptitude. Journal of Experimental Psychology: General, 141(4), 788-798. https://doi.org/10.1037/a0027968

Muris, P., \& van der Heiden, S. (2006). Anxiety, depression, and judgments about the probability of future negative and positive events in children. Journal of Anxiety Disorders, 20(2), 252-261. https://doi.org/10.1016/j.janxdis.2004.12.001 
Muthén, B., \& Muthén, L. (1998). Mplus User's Guide. Muthén \& Muthén.

Nahrgang, J. D., Morgeson, F. P., \& Hofmann, D. A. (2011). Safety at work: A meta-analytic investigation of the link between job demands, job resources, burnout, engagement, and safety outcomes. Journal of Applied Psychology, 96(1), 71-94. https://doi.org/10.1037/a0021484

Nawijn, J., de Bloom, J., \& Geurts, S. A. E. (2013). Pre-vacation time: Blessing or burden? Leisure Sciences, 35(1), 33-44. https://doi.org/10.1080/01490400.2013.739875

Newby-Clark, I. R., \& Ross, M. (2003). Conceiving the past and future. Personality \& Social Psychology Bulletin, 29(7), 807-818. https://doi.org/10.1177/0146167203029007001

Newman, D. B., Tay, L., \& Diener, E. (2014). Leisure and subjective well-being: A model of psychological mechanisms as mediating factors. Journal of Happiness Studies, 15(3), 555-578. https://doi.org/10.1007/s10902-013-9435-x

Nezlek, J. B. (2017). A practical guide to understanding reliability in studies of within-person variability. Journal of Research in Personality, 69, 149-155. https://doi.org/10.1016/j.jrp.2016.06.020

Nicholson, T., \& Griffin, B. (2017). Thank goodness it's Friday: Weekly pattern of workplace incivility. Anxiety, Stress, and Coping, 30(1), 1-14. https://doi.org/10.1080/10615806.2016.1192150

Nielsen, K., Nielsen, M. B., Ogbonnaya, C., Känsälä, M., Saari, E., \& Isaksson, K. (2017). Workplace resources to improve both employee well-being and performance: A systematic review and meta-analysis. Work \& Stress, 31(2), 101-120. https://doi.org/10.1080/02678373.2017.1304463

Niessen, C., \& Jimmieson, N. L. (2016). Threat of resource loss: The role of self-regulation in adaptive task performance. Journal of Applied Psychology, 101(3), 450-462. https://doi.org/10.1037/ap10000049

Oerlemans, W. G., Bakker, A. B., \& Demerouti, E. (2014). How feeling happy during off-job activities helps successful recovery from work: A day reconstruction study. Work \& Stress, 35, 1-19. https://doi.org/10.1080/02678373.2014.901993

Oettingen, G. (2012). Future thought and behaviour change. European Review of Social Psychology, 23(1), 1-63. https://doi.org/10.1080/10463283.2011.643698

Oettingen, G., Pak, H., \& Schnetter, K. (2001). Self-regulation of goal-setting: Turning free fantasies about the future into binding goals. Journal of Personality and Social Psychology, 80(5), 736-753. https://doi.org/10.1037//0022-3514.80.5.736 
Ohly, S., Sonnentag, S., Niessen, C., \& Zapf, D. (2010). Diary Studies in Organizational Research. Journal of Personnel Psychology, 9(2), 79-93. https://doi.org/10.1027/1866-5888/a000009

Organ, D. W. (1988). A restatement of the satisfaction-performance hypothesis. Journal of Management, 14(4), 547-557. https://doi.org/10.1177/014920638801400405

Organ, D. W. (2018). Organizational citizenship behavior: Recent trends and developments. Annual Review of Organizational Psychology and Organizational Behavior, 5(1), 295-306. https://doi.org/10.1146/annurev-orgpsych-032117-104536

Orth, M., \& Volmer, J. (2017). Daily within-person effects of job autonomy and work engagement on innovative behaviour: The cross-level moderating role of creative selfefficacy. European Journal of Work and Organizational Psychology, 26(4), 601-612. https://doi.org/10.1080/1359432X.2017.1332042

Ouyang, K., Cheng, B. H., Lam, W., \& Parker, S. K. (2019). Enjoy your evening, be proactive tomorrow: How off-job experiences shape daily proactivity. Journal of Applied Psychology, 104(8), 1003-1019. https://doi.org/10.1037/apl0000391

Park, H. I., \& Lee, H. (2015). The effects of recovery-related self-efficacy on occupational health among Korean workers. International Journal of Stress Management, 22(4), 372-394. https://doi.org/10.1037/a0039185

Park, Y., \& Kim, S. (2019). Customer mistreatment harms nightly sleep and next-morning recovery: Job control and recovery self-efficacy as cross-level moderators. Journal of Occupational Health Psychology, 24(2), 256-269. https://doi.org/10.1037/ocp0000128

Park, Y., \& Sprung, J. M. (2015). Weekly work-school conflict, sleep quality, and fatigue: Recovery self-efficacy as a cross-level moderator. Journal of Organizational Behavior, 36(1), 112-127. https://doi.org/10.1002/job.1953

Parke, M. R., Weinhardt, J. M., Brodsky, A., Tangirala, S., \& DeVoe, S. E. (2018). When daily planning improves employee performance: The importance of planning type, engagement, and interruptions. Journal of Applied Psychology, 103(3), 300-312. https://doi.org/10.1037/ap10000278

Parker, S. L., Sonnentag, S., Jimmieson, N. L., \& Newton, C. J. (2020). Relaxation during the evening and next-morning energy: The role of hassles, uplifts, and heart rate variability during work. Journal of Occupational Health Psychology, 25(2), 83-98. https://doi.org/10.1037/ocp0000155 
Parker, S. R., \& Smith, M. A. (1976). Work and leisure. In R. Dubin (Ed.), Handbook of work, organization and society (pp. 37-64). Rand McNally.

Peifer, C., Syrek, C., Ostwald, V., Schuh, E., \& Antoni, C. H. (2019). Thieves of flow: How unfinished tasks at work are related to flow experience and wellbeing. Journal of Happiness Studies, 11(2), 205. https://doi.org/10.1007/s10902-019-00149-z

Peterson, C. (2000). The future of optimism. American Psychologist, 55(1), 44-55. https://doi.org/10.1037//0003-066X.55.1.44

Petrou, P., Bakker, A. B., \& van den Heuvel, M. (2017). Weekly job crafting and leisure crafting: Implications for meaning-making and work engagement. Journal of Occupational and Organizational Psychology, 90(2), 129-152. https://doi.org/10.1111/joop.12160

Pinheiro, J. C., \& Bates, D. M. (2000). Linear mixed-effects models: basic concepts and examples. Springer.

Podsakoff, P. M., MacKenzie, S. B., Lee, J.-Y., \& Podsakoff, N. P. (2003). Common method biases in behavioral research: A critical review of the literature and recommended remedies. Journal of Applied Psychology, 88(5), 879-903. https://doi.org/10.1037/0021-9010.88.5.879

Podsakoff, P. M., MacKenzie, S. B., \& Podsakoff, N. P. (2012). Sources of method bias in social science research and recommendations on how to control it. Annual Review of Psychology, 63, 539-569. https://doi.org/10.1146/annurev-psych-120710-100452

Preacher, K. J., Curran, P. J., \& Bauer, D. J. (2006). Computational tools for probing interactions in multiple linear regression, multilevel modeling, and latent curve analysis. Journal of Educational and Behavioral Statistics, 31(4), 437-448. https://doi.org/10.3102/10769986031004437

Preacher, K. J., Zyphur, M. J., \& Zhang, Z. (2010). A general multilevel SEM framework for assessing multilevel mediation. Psychological Methods, 15(3), 209-233. https://doi.org/10.1037/a0020141

Querstret, D., \& Cropley, M. (2012). Exploring the relationship between work-related rumination, sleep quality, and work-related fatigue. Journal of Occupational Health Psychology, 17(3), 341-353. https://doi.org/10.1037/a0028552

Querstret, D., Cropley, M., \& Fife-Schaw, C. (2017). Internet-based instructor-led mindfulness for work-related rumination, fatigue, and sleep: Assessing facets of mindfulness as mechanisms of change. A randomized waitlist control trial. Journal of 
Occupational Health Psychology, 22(2), 153-169.

https://doi.org/10.1037/ocp0000028

Quoidbach, J., Wood, A. M., \& Hansenne, M. (2009). Back to the future: the effect of daily practice of mental time travel into the future on happiness and anxiety. The Journal of Positive Psychology, 4(5), 349-355. https://doi.org/10.1080/17439760902992365

Ragsdale, J. M., \& Beehr, T. A. (2016). A rigorous test of a model of employees' resource recovery mechanisms during a weekend. Journal of Organizational Behavior, 37(6), 911-932. https://doi.org/10.1002/job.2086

Ragsdale, J. M., Beehr, T. A., Grebner, S., \& Han, K. (2011). An integrated model of weekday stress and weekend recovery of students. International Journal of Stress Management, 18(2), 153-180. https://doi.org/10.1037/a0023190

Randall, J. G., Oswald, F. L., \& Beier, M. E. (2014). Mind-wandering, cognition, and performance: A theory-driven meta-analysis of attention regulation. Psychological Bulletin, 140(6), 1411-1431. https://doi.org/10.1037/a0037428

Raudenbush, S. W., \& Bryk, A. S. (2002). Hierarchical linear models: Applications and data analysis methods. Sage.

Revelle, W. (2020). psych: Procedures for personality and psychological research.

Northwestern University. R package version 2.0.8, https://CRAN.Rproject.org/package $=$ psych

Rook, J. W., \& Zijlstra, F. R. H. (2006). The contribution of various types of activities to recovery. European Journal of Work and Organizational Psychology, 15(2), 218-240. https://doi.org/10.1080/13594320500513962

Roseman, I. J. (1996). Appraisal determinants of emotions: Constructing a more accurate and comprehensive theory. Cognition \& Emotion, 10(3), 241-278. https://doi.org/10.1080/026999396380240

Russell, J. A. (1980). A circumplex model of affect. Journal of Personality and Social Psychology, 39(6), 1161-1178. https://doi.org/10.1037/h0077714

Salanova, M., Schaufeli, W. B., Xanthopoulou, D., \& Bakker, A. B. (2010). The gain spiral of resoruces and work engagement: Sustaining positive worklife. In M. P. Leiter \& A. B. Bakker (Eds.), Work engagement: A handbook of essential theory and research (pp. 118-131). Taylor \& Francis.

Schaufeli, W. B., \& Bakker, A. B. (2004). Job demands, job resources, and their relationship with burnout and engagement: A multi-sample study. Journal of Organizational Behavior, 25(3), 293-315. https://doi.org/10.1002/job.248 
Schaufeli, W. B., Bakker, A. B., \& Salanova, M. (2006a). The measurement of work engagement with a short questionnaire. Educational and Psychological Measurement, 66(4), 701-716. https://doi.org/10.1177/0013164405282471

Schaufeli, W. B., Bakker, A. B., \& Salanova, M. (2006b). The Measurement of work engagement with a short questionnaire: A cross-national study. Educational and Psychological Measurement, 66(4), 701-716. https://doi.org/10.1177/0013164405282471

Schaufeli, W. B., Martínez, I. M., Pinto, A. M., Salanova, M., \& Bakker, A. B. (2002). Burnout and engagement in university students. Journal of Cross-Cultural Psychology, 33(5), 464-481. https://doi.org/10.1177/0022022102033005003

Schaufeli, W. B., Salanova, M., González-romá, V., \& Bakker, A. B. (2002). The measurement of engagement and burnout: A two sample confirmatory factor analytic approach. Journal of Happiness Studies, 3(1), 71-92. https://doi.org/10.1023/A:1015630930326

Scheier, M. F., \& Carver, C. S. (1985). Optimism, coping, and health: Assessment and implications of generalized outcome expectancies. Health Psychology, 4(3), 219-247. https://doi.org/10.1037//0278-6133.4.3.219

Schmitt, A., Gielnik, M. M., \& Seibel, S. (2019). When and how does anger during goal pursuit relate to goal achievement? The roles of persistence and action planning. Motivation and Emotion, 43, 205-217. https://doi.org/10.1007/s11031-018-9720-4

Schneider, B., Yost, A. B., Kropp, A., Kind, C., \& Lam, H. (2018). Workforce engagement: What it is, what drives it, and why it matters for organizational performance. Journal of Organizational Behavior, 39(4), 462-480. https://doi.org/10.1002/job.2244

Seibel, S., Volmer, J., \& Syrek, C. J. (2020). Get a taste of your leisure time: the relationship between leisure thoughts, pleasant anticipation, and work engagement. European Journal of Work and Organizational Psychology, 29(6), 889-906. https://doi.org/10.1080/1359432X.2020.1804875

Semmer, N., Zapf, D., \& Dunckel, H. (1999). Instrument zur stressbezogenen Tätigkeitsanalyse [Stress-oriented task analysis]. In H. Dunckel (Ed.), Handbuch psychologischer Arbeitsanalyseverfahren (pp. 179-204). Hochschulverlag an der ETH.

Seo, M.-G., Barrett, L. F., \& Bartunek, J. M. (2004). The role of affective experience in work motivation. Academy of Management Review, 29(3), 423. https://doi.org/10.2307/20159052 
Sheldon, K. M., \& Lyubomirsky, S. (2006). How to increase and sustain positive emotion: The effects of expressing gratitude and visualizing best possible selves. The Journal of Positive Psychology, 1(2), 73-82. https://doi.org/10.1080/17439760500510676

Shrout, P. E., \& Lane, S. P. (2012). Psychometrics. In M. R. Mehl \& T. S. Conner (Eds.), Handbook of research methods for studying daily life (pp. 302-320). The Guilford Press.

Shuck, B. (2011). Integrative literature review: Four emerging perspectives of employee engagement: An integrative literature review. Human Resource Development Review, 10(3), 304-328. https://doi.org/10.1177/1534484311410840

Siu, O. L., Cooper, C. L., \& Phillips, D. R. (2014). Intervention studies on enhancing work well-being, reducing burnout, and improving recovery experiences among Hong Kong health care workers and teachers. International Journal of Stress Management, 21(1), 69-84. https://doi.org/10.1037/a0033291

Smallwood, J., \& Schooler, J. W. (2013). The restless mind. Psychology of Consciousness: Theory, Research, and Practice, 1(S), 130-149. https://doi.org/10.1037/23265523.1.S. 130

Smallwood, J., \& Schooler, J. W. (2015). The science of mind wandering: Empirically navigating the stream of consciousness. Annual Review of Psychology, 66, 487-518. https://doi.org/10.1146/annurev-psych-010814-015331

Smith, A. P. (2013). Time of day and performance. In A. P. Smith \& D. M. Jones (Eds.), State and Trait. Human Performance (3rd ed., Vol. 3, pp. 217-237). Academic Press.

Smith, J. L., \& Bryant, F. B. (2013). Are we having fun yet? Savoring, Type A behavior, and vacation enjoyment. International Journal of Wellbeing, 3(1), 1-19. https://doi.org/10.5502/ijw.v3i1.1

Smith, J. L., \& Hanni, A. A. (2019). Effects of a Savoring Intervention on Resilience and Well-Being of Older Adults. Journal of Applied Gerontology, 38(1), 137-152. https://doi.org/10.1177/0733464817693375

Smith, J. L., Harrison, P. R., Kurtz, J. L., \& Bryant, F. B. (2014). Nurturing the capacity to savor: Interventions to enhance the enjoyment of positive experiences. In A. C. Parks \& Schueller Jennifer L. (Eds.), The wiley blackwell handbook of positive psychological interventions (pp. 42-65). John Wiley \& Sons.

Snijders, T. A. B., \& Bosker, R. J. (2012). Multilevel analysis: An introduction to basic and advanced multilevel modeling (2nd edition). Sage. 
Sonnentag, S. (2001). Work, recovery activities, and individual well-being: A diary study. Journal of Occupational Health Psychology, 6, 196-210. https://doi.org/10.1037/1076-8998.6.3.196

Sonnentag, S. (2003, June). Recovery, work engagement, and proactive behavior: A new look at the interface between nonwork and work. Journal of Applied Psychology, 88(3), 518-528. https://doi.org/10.1037/0021-9010.88.3.518

Sonnentag, S. (2018). The recovery paradox: Portraying the complex interplay between job stressors, lack of recovery, and poor well-being. Research in Organizational Behavior, 38, 169-185. https://doi.org/10.1016/j.riob.2018.11.002

Sonnentag, S., \& Bayer, U.-V. (2005). Switching off mentally: Predictors and consequences of psychological detachment from work during off-job time. Journal of Occupational Health Psychology, 10(4), 393-414. https://doi.org/10.1037/1076-8998.10.4.393

Sonnentag, S., Binnewies, C., \& Mojza, E. J. (2008). "Did you have a nice evening?" A daylevel study on recovery experiences, sleep, and affect. Journal of Applied Psychology, 93(3), 674-684. https://doi.org/10.1037/0021-9010.93.3.674

Sonnentag, S., Dormann, C., \& Demerouti, E. (2010). Not all days are created equal: The concept of state work engagement. In M. P. Leiter \& A. B. Bakker (Eds.), Work engagement: A handbook of essential theory and research (pp. 25-38). Taylor \& Francis.

Sonnentag, S., Eck, K., Fritz, C., \& Kühnel, J. (2019). Morning Reattachment to Work and Work Engagement During the Day: A Look at Day-Level Mediators. Journal of Management, 1(3), 014920631982982. https://doi.org/10.1177/0149206319829823

Sonnentag, S., \& Fritz, C. (2007). The recovery experience questionnaire: Development and validation of a measure for assessing recuperation and unwinding from work. Journal of Occupational Health Psychology, 12(3), 204-221. https://doi.org/10.1037/10768998.12.3.204

Sonnentag, S., \& Fritz, C. (2015). Recovery from job stress: The stressor-detachment model as an integrative framework. Journal of Organizational Behavior, 36(S1), S72-S103. https://doi.org/10.1002/job.1924

Sonnentag, S., \& Geurts, S. A. E. (2009). Methodological issues in recovery research. In S. Sonnentag, P. L. Perrewé, \& D. C. Ganster (Eds.), Current perspectives on job-stress recovery: Research in occupational stress and well being (pp. 1-36). Emerald Group. https://doi.org/10.1108/S1479-3555(2009)0000007004 
Sonnentag, S., \& Kruel, U. (2006). Psychological detachment from work during off-job time: The role of job stressors, job involvement, and recovery-related self-efficacy. European Journal of Work and Organizational Psychology, 15(2), 197-217. https://doi.org/10.1080/13594320500513939

Sonnentag, S., \& Kühnel, J. (2016). Coming back to work in the morning: Psychological detachment and reattachment as predictors of work engagement. Journal of Occupational Health Psychology, 21(4), 379-390. https://doi.org/10.1037/ocp0000020

Sonnentag, S., Mojza, E. J., Binnewies, C., \& Scholl, A. (2008). Being engaged at work and detached at home: A week-level study on work engagement, psychological detachment, and affect. Work \& Stress, 22(3), 257-276. https://doi.org/10.1080/02678370802379440

Sonnentag, S., Venz, L., \& Casper, A. (2017). Advances in recovery research: What have we learned? What should be done next? Journal of Occupational Health Psychology, 22(3), 365-380. https://doi.org/10.1037/ocp0000079

Speier, C., Valacich, J. S., \& Vessey, I. (1999). The influence of task interruption on individual decision making: An information overload perspective. Decision Sciences, 30(2), 337-360. https://doi.org/10.1111/j.1540-5915.1999.tb01613.x

Stebbins, R. A. (2018). Leisure as not work: a (far too) common definition in theory and research on free-time activities. World Leisure Journal, 60(4), 255-264. https://doi.org/10.1080/16078055.2018.1517107

Steed, L. B., Swider, B. W., Keem, S., \& Liu, J. T. (2019). Leaving Work at Work: A MetaAnalysis on Employee Recovery From Work. Journal of Management, 014920631986415. https://doi.org/10.1177/0149206319864153

Stone, A. A., Schneider, S., \& Harter, J. K. (2012). Day-of-week mood patterns in the United States: On the existence of 'Blue Monday', 'Thank God it's Friday' and weekend effects. The Journal of Positive Psychology, 7(4), 306-314. https://doi.org/10.1080/17439760.2012.691980

Syrek, C. J., Kühnel, J., Vahle-Hinz, T., \& de Bloom, J. (2018). Share, like, twitter, and connect: Ecological momentary assessment to examine the relationship between nonwork social media use at work and work engagement. Work \& Stress, 32(3), 209-227. https://doi.org/10.1080/02678373.2017.1367736 
Syrek, C. J., Weigelt, O., Kühnel, J., \& de Bloom, J. (2018). All I want for Christmas is recovery - changes in employee affective well-being before and after vacation. Work \& Stress, 32(4), 313-333. https://doi.org/10.1080/02678373.2018.1427816

Szpunar, K. K. (2010). Episodic future thought: An emerging concept. Perspectives on Psychological Science, 5(2), 142-162. https://doi.org/10.1177/1745691610362350

ten Brummelhuis, L. L., \& Bakker, A. B. (2012a). A resource perspective on the work-home interface: The work-home resources model. American Psychologist, 67(7), 545-556. https://doi.org/10.1037/a0027974

ten Brummelhuis, L. L., \& Bakker, A. B. (2012b). Staying engaged during the week: The effect of off-job activities on next day work engagement. Journal of Occupational Health Psychology, 17(4), 445-455. https://doi.org/10.1037/a0029213

Thiart, H., Lehr, D., Ebert, D. D., Sieland, B., Berking, M., \& Riper, H. (2013). Log in and breathe out: Efficacy and cost-effectiveness of an online sleep training for teachers affected by work-related strain--study protocol for a randomized controlled trial. Trials, 14, 169. https://doi.org/10.1186/1745-6215-14-169

Thierry, H., \& Janson, B. (1998). Work time and behavior at work. In P. J. D. Drenth, H. Thierry, \& C. J. de Wolff (Eds.), A handbook of work and organizational psychology (2nd ed., Vol. 2, pp. 5-33). Taylor \& Francis.

Thompson, M. S., \& Cooper, C. L. (2001). A rose by any other name...: A commentary on Hobfoll's Conservation of Resources theory. Applied Psychology: An International Review, 50(3), 408-418.

Tims, M., Bakker, A. B., \& Xanthopoulou, D. (2011). Do transformational leaders enhance their followers' daily work engagement? The Leadership Quarterly, 22(1), 121-131. https://doi.org/10.1016/j.leaqua.2010.12.011

Tivadar, R. I., \& Murray, M. M. (2019). A Primer on Electroencephalography and EventRelated Potentials for Organizational Neuroscience. Organizational Research Methods, 22(1), 69-94. https://doi.org/10.1177/1094428118804657

Trope, Y., \& Liberman, N. (2010). Construal-level theory of psychological distance. Psychological Review, 117(2), 440-463. https://doi.org/10.1037/a0018963

Trougakos, J. P., \& Hideg, I. (2009). Momentary work recovery: The role of within-day work breaks. In S. Sonnentag, P. L. Perrewé, \& D. C. Ganster (Eds.), Current perspectives on job-stress recovery: Research in occupational stress and well being (Vol. 7, pp. 37-84). Emerald Group. https://doi.org/10.1108/S1479-3555(2009)0000007005 
van Boven, L., \& Ashworth, L. (2007). Looking forward, looking back: Anticipation is more evocative than retrospection. Journal of Experimental Psychology: General, 136(2), 289-300. https://doi.org/10.1037/0096-3445.136.2.289

van den Heuvel, M., Demerouti, E., Bakker, A. B., \& Schaufeli, W. B. (2010). Personal resources and work engagement in the face of change. In J. Houdmont \& S. Leka (Eds.), Contemporary occupational health psychology: Global perspectives on research and practice (pp. 124-150). John Wiley \& Sons. https://doi.org/10.1002/9780470661550.ch7

van Hooff, M. L. M., \& Pater, I. E. de (2017). Let's have fun tonight: The role of pleasure in daily recovery from work. Applied Psychology: An International Review, 66(3), 359381. https://doi.org/10.1111/apps. 12098

van Quaquebeke, N., Kerschreiter, R., Buxton, A. E., \& van Dick, R. (2010). Two lighthouses to navigate: Effects of ideal and counter-ideal values on follower identification and satisfaction with their leaders. Journal of Business Ethics, 93(2), 293-305. https://doi.org/10.1007/s10551-009-0222-X

Volman, F. E., Bakker, A. B., \& Xanthopoulou, D. (2013). Recovery at home and performance at work: A diary study on self-family facilitation. European Journal of Work and Organizational Psychology, 22(2), 218-234. https://doi.org/10.1080/1359432X.2011.648375

Volmer, J. (2015). Followers' daily reactions to social conflicts with supervisors: The moderating role of core self-evaluations and procedural justice perceptions. The Leadership Quarterly, 26(5), 719-731. https://doi.org/10.1016/j.leaqua.2015.01.005

Volmer, J., Binnewies, C., Sonnentag, S., \& Niessen, C. (2012). Do social conflicts with customers at work encroach upon our private lives? A diary study. Journal of Occupational Health Psychology, 17(3), 304-315. https://doi.org/10.1037/a0028454

Voss, J. (1967). The definition of leisure. Journal of Economic Issues, 1(1-2), 91-106. https://doi.org/10.1080/00213624.1967.11502742

Waldman, D. A., Wang, D., \& Fenters, V. (2019). The added value of neuroscience methods in organizational research. Organizational Research Methods, 22(1), 223-249. https://doi.org/10.1177/1094428116642013

Wallace, J. C., \& Chen, G. (2005). Development and validation of a work-specific measure of cognitive failure: Implications for occupational safety. Journal of Occupational and Organizational Psychology, 78(4), 615-632. https://doi.org/10.1348/096317905X37442 
Wallace, J. C., Edwards, B. D., Shull, A., \& Finch, D. M. (2009). Examining the consequences in the tendency to suppress and reappraise emotions on task-related job performance. Human Performance, 22(1), 23-43. https://doi.org/10.1080/08959280802540957

Wang, M., Liu, S., Liao, H., Gong, Y., Kammeyer-Mueller, J., \& Shi, J. (2013). Can't get it out of my mind: Employee rumination after customer mistreatment and negative mood in the next morning. Journal of Applied Psychology, 98(6), 989-1004. https://doi.org/10.1037/a0033656

Wanous, J. P., Reichers, A. E., \& Hudy, M. J. (1997). Overall job satisfaction: How good are single-item measures? Journal of Applied Psychology, 82(2), 247-252. https://doi.org/10.1037/0021-9010.82.2.247

Watkins, E. R. (2008). Constructive and unconstructive repetitive thought. Psychological Bulletin, 134(2), 163-206. https://doi.org/10.1037/0033-2909.134.2.163

Weigelt, O., Syrek, C. J., Schmitt, A., \& Urbach, T. (2019a). Finding peace of mind when there still is so much left undone-A diary study on how job stress, competence need satisfaction, and proactive work behavior contribute to work-related rumination during the weekend. Journal of Occupational Health Psychology, 24(3), 373-386. https://doi.org/10.1037/ocp0000117

Weigelt, O., Syrek, C. J., Schmitt, A., \& Urbach, T. (2019b). Finding peace of mind when there still is so much left undone-A diary study on how job stress, competence need satisfaction, and proactive work behavior contribute to work-related rumination during the weekend. Journal of Occupational Health Psychology, 24(3), 373-386. https://doi.org/10.1037/ocp0000117

Wheeler, A. R., Shanine, K. K., Leon, M. R., \& Whitman, M. V. (2014). Student-recruited samples in organizational research: A review, analysis, and guidelines for future research. Journal of Occupational and Organizational Psychology, 87(1), 1-26. https://doi.org/10.1111/joop.12042

Wiesenfeld, B. M., Reyt, J.-N., Brockner, J., \& Trope, Y. (2017). Construal level theory in organizational research. Annual Review of Organizational Psychology and Organizational Behavior, 4(1), 367-400. https://doi.org/10.1146/annurev-orgpsych032516-113115

Xanthopoulou, D., Bakker, A. B., Demerouti, E., \& Schaufeli, W. B. (2007). The role of personal resources in the job demands-resources model. International Journal of Stress Management, 14(2), 121-141. https://doi.org/10.1037/1072-5245.14.2.121 
Xanthopoulou, D., Bakker, A. B., Demerouti, E., \& Schaufeli, W. B. (2009). Work engagement and financial returns: A diary study on the role of job and personal resources. Journal of Occupational and Organizational Psychology, 82(1), 183-200. https://doi.org/10.1348/096317908X285633

Xanthopoulou, D., Bakker, A. B., Heuven, E., Demerouti, E., \& Schaufeli, W. B. (2008). Working in the sky: A diary study on work engagement among flight attendants. Journal of Occupational Health Psychology, 13(4), 345-356. https://doi.org/10.1037/1076-8998.13.4.345

Xu, Z., \& Guo, H. (2019). Advantages of anticipated emotions over anticipatory emotions and cognitions in health decisions: A meta-analysis. Health Communication, 34(7), 774781. https://doi.org/10.1080/10410236.2018.1434738

Yang, F., Lu, M., \& Huang, X. (2020). Customer mistreatment and employee well-being: A daily diary study of recovery mechanisms for frontline restaurant employees in a hotel. International Journal of Hospitality Management, 91, 102665.

https://doi.org/10.1016/j.ijhm.2020.102665 